Алгебра и анализ

Tом. 18 (2006), № 3
St. Petersburg Math. J.

Vol. 18 (2007), No. 3, Pages 459-510

S 1061-0022(07)00957-0

Article electronically published on April 11, 2007

\title{
ELASTODYNAMICS IN DOMAINS WITH EDGES
}

\author{
S. I. MATYUKEVICH AND B. A. PLAMENEVSKII
}

\begin{abstract}
Time-dependent boundary value problems with given displacements or stresses on the boundary of a domain are considered. The purpose is to describe the asymptotics of solutions near the edges of the boundary (including formulas for the "stress intensity factors"). The approach is based on various (energy and weighted) estimates of solutions. The weighted estimates in question are mixed in the sense that, in distinct zones, they involve derivatives of different orders. The method is implemented for problems in the cylinder $\mathbb{D} \times \mathbb{R}$, where $\mathbb{D}$ is an $m$-dimensional wedge, $m \geq 2$, and $\mathbb{R}$ is the time axis. For the cylinder $G \times \mathbb{R}$, where $G$ is a bounded domain with edges on the boundary, all the steps of the method are described except for the final one, which is related to the asymptotics itself. This step consists in compiling some known results of the theory of elliptic boundary value problems.
\end{abstract}

\section{Contents}

$\S 1 . \quad$ Introduction

$\S 2$. Homogeneous energy estimates for solutions of boundary value problems with parameter in a wedge

$\S 3$. Nonhomogeneous energy estimates for solutions of boundary value problems with parameter in a wedge

$\S 4$. Strong solutions

$\S 5$. Weighted a priori estimates for solutions of boundary value problems with parameter in a wedge

$\S 6$. Boundary value problem in a cone in a scale of weighted spaces

$\S 7$. On the time-dependent problem in a wedge

$\S 8$. Energy estimates for solutions in a bounded domain

$\S 9$. Weighted estimates in a bounded domain with edge

References

\section{§1. INTRODUCTION}

Let $G$ be a domain in $\mathbb{R}^{m}$ with compact closure $\bar{G}, m \geq 2$. The boundary $\partial G$ may contain nonintersecting closed smooth "edges" of various dimensions. In the cylinder $\{(x, t): x \in G,-\infty<t<+\infty\}$, we study the equations of elastodynamics. The Dirichlet condition (displacements) or the Neumann condition (stresses) is given on the boundary $\partial G \times \mathbb{R}$ of the cylinder. Our goal is to describe the behavior of the solutions near the edges.

2000 Mathematics Subject Classification. Primary 35L30, 35L35.

Key words and phrases. Weighted estimates, asymptotics of solutions near edges, stress intensity factors.

Supported by RFBR (grant no. 05-01-01077). 
In the paper, we continue the investigations presented in [1]-[3]. In contrast to [2] and 3], where, in particular, some problems were discussed for the time-dependent Lamé system, here the nonisotropic equations of elasticity are considered. A number of results are new for the Lamé system as well. On the other hand, some theorems obtained in [2] and [3] can be extended to the general case with evident modification. To make the exposition more coherent, we present all the theory developed to date but pass to the survey style wherever appropriate references are possible. (The basic results are listed in the Introduction, including the results new for the Lamé system.)

We develop a method for deriving asymptotic formulas for solutions near the edges of the boundary. This method was suggested in [1] for the wave operator $\partial_{t}^{2}-\Delta_{x}$ and applied in [2] and [3] to a class of hyperbolic systems. The stage for the description of the asymptotics is set by obtaining various estimates of solutions, the study of model problems, etc. At the final step, some results known in the theory of elliptic problems in domains with edges on the boundary are applied. In the paper, all the ingredients of the method (including the final step mentioned above) are implemented for problems in the cylinder $\{(x, t): x \in \mathbb{D},-\infty<t<+\infty\}$, where $\mathbb{D}$ is the wedge $\mathbb{K} \times \mathbb{R}^{m-n}, n \geq 2$, and $\mathbb{K}$ is an open cone in $\mathbb{R}^{n}$ smooth outside of the vertex. All the preparatory work is done also for the general case (with the wedge $\mathbb{D}$ replaced by a bounded domain $G$ having edges on the boundary $\partial G$ ). However, the final step (derivation of the asymptotics itself) is not presented here for the general case. Though long enough, this step is, in essence, a compilation and consists in generalizing some fragments of the elliptic theory.

Let us describe some results; for the time being, we restrict ourselves to a domain $G$ with a conical point and to the Dirichlet boundary conditions. Assume that the conical point coincides with the coordinate origin $\mathcal{O}$. We consider the problem

$$
\begin{aligned}
\mathcal{L}\left(x, D_{x}, D_{t}\right) u(x, t) & =f(x, t), & & (x, t) \in G \times \mathbb{R}, \\
u(x, t) & =0, & & (x, t) \in \partial G \times \mathbb{R},
\end{aligned}
$$

with the matrix differential operator

$$
\left(L\left(x, D_{x}, D_{t}\right) u\right)_{p}(x, t)=\partial_{t}^{2} u(x, t)-\partial_{k}\left(a_{p q}^{k l}(x) \partial_{l} u_{q}(x, t)\right),
$$

where $\partial_{k}=\partial / \partial x_{k}, D_{x_{k}}=-i \partial_{k}$, etc., $p=1, \ldots, m$, and summation is over $1 \leq q, k$, $l \leq m$. The entries $a_{p q}^{k l}$ of the Hooke tensor are real-valued functions in $C^{\infty}(\bar{G})$; the usual symmetry relations

are assumed, as well as the condition

$$
a_{p q}^{k l}=a_{q p}^{l k}, \quad a_{p q}^{k l}=a_{k q}^{p l}
$$

$$
\sum_{k, l, p, q} a_{p q}^{k l}(x) \xi_{k}^{p} \xi_{l}^{q} \geq c \sum_{k, p}\left(\xi_{k}^{p}\right)^{2}
$$

in $\bar{G}$ for the symmetric matrices, $\xi_{k}^{p}=\xi_{p}^{k}$; the numbers $\xi_{p}^{k}$ are real and the constant $c$ is positive.

The external force $f$ in (10) is subject to the condition

$$
\int_{G} \int_{\mathbb{R}} e^{-2 \gamma t}|f(x, t)|^{2} \mathrm{~d} x \mathrm{~d} t<\infty,
$$

where $\gamma$ is a sufficiently large positive number. There exists a unique "strong" solution of problem (1), (2), satisfying

$$
\int_{G} \int_{\mathbb{R}} e^{-2 \gamma t}\left(\left|\nabla_{x, t} u(x, t)\right|^{2}+|u(x, t)|^{2}\right) \mathrm{d} x \mathrm{~d} t \leq c \int_{G} \int_{\mathbb{R}} e^{-2 \gamma t}|f(x, t)|^{2} \mathrm{~d} x \mathrm{~d} t .
$$

Note that the problem of the form (1), (2) in the semicylinder $G \times \mathbb{R}_{+}$with zero initial conditions at $t=0$ reduces to the problem in the cylinder $G \times \mathbb{R}$. 
To obtain the asymptotics of the solution in a neighborhood of the conical point, we need to impose some additional requirements on $f$ in (1). For simplicity, we suppose that $\operatorname{supp} f$ is a compact set in $(\bar{G} \backslash \mathcal{O}) \times \mathbb{R}$, so it is "always" separated away from the conical point. Also, we assume that, near the conical point $\mathcal{O}$, the domain $G$ coincides with a cone $\mathbb{K}$ smooth outside of the vertex. Then the solution $u$ admits the representation

$$
u(x, t) \sim \sum_{j \geq 0} c_{j}(t) u_{j}(x, t)
$$

as $x \rightarrow 0$. Here, in its turn, $u_{j}$ is an asymptotic series as $x \rightarrow 0$ whose principal term $v_{j}$ is independent of $t$ and solves the homogeneous elliptic problem

$$
\begin{aligned}
\mathcal{L}\left(0, D_{x}, 0\right) v(x)=0, & x \in \mathbb{K}, \\
v(x)=0, & x \in \partial \mathbb{K} .
\end{aligned}
$$

As $x \rightarrow 0$, we have $v_{j}(x)=O\left(|x|^{\mu_{j}}\right)$, where $\mu_{j} \rightarrow+\infty$. (The above asymptotic expansions are described in $\S \S 6$ and 7 . In particular, in $\S 7$ we obtain an estimate for the remainder of the series (4), thus making the meaning of (4) more precise.) The series $u_{j}$ is determined completely by the data of problem (1), (2) taken in an arbitrarily small neighborhood of the conical point $\mathcal{O}$; in this sense, $u_{j}$ is a "local" element of the asymptotics. The coefficients $c_{j}$ in (4) satisfy

$$
c_{j}(t)=\int_{G} \int_{\mathbb{R}} f(x, t) \overline{w_{j}}(x, t-s) \mathrm{d} x \mathrm{~d} s ;
$$

the functions $(x, t) \mapsto w_{j}(x, t)$ are solutions of the homogeneous problem (11), (2), and they are uniquely determined by their (growing) asymptotics as $x \rightarrow 0$. Clearly, the coefficients $c_{j}$ are "global" elements of the asymptotics.

We do not study the properties of the $w_{j}$ in this paper. However, in 4, 5, for the wave operator $\mathcal{L}\left(D_{x}, D_{t}\right)=\partial_{t}^{2}-\Delta_{x}$ in a cone $\mathbb{K}$, some explicit expressions for $w_{j}$ were obtained in terms of special functions. Such expressions show that

$$
\begin{aligned}
\operatorname{supp} w_{j} & \subset\{(x, t):|x| \leq t\}, \\
\operatorname{sing} \operatorname{supp} w_{j} & \subset\{(x, t):|x|=t\},
\end{aligned}
$$

where $\operatorname{sing} \operatorname{supp} w_{j}$ is the singular support of $w_{j}$. Let us use this example to interpret (4) and (5).

We assume for a while that $\mathcal{L}\left(D_{x}, D_{t}\right)=\partial_{t}^{2}-\Delta_{x}$ and $G=\mathbb{K}$. By (6) , the integrand in (5) vanishes outside the intersection of $\mathbb{K} \times \mathbb{R}$ and the light cone $P_{t}$ going to the past and with vertex at $(0, t)$. Suppose as before that $\operatorname{supp} f$ is a compact subset of $\mathbb{K} \times \mathbb{R}$. Let $a$ and $b$ be moments such that $P_{t} \cap \operatorname{supp} f=\varnothing$ for $t<a$ and $\operatorname{supp} f \subset P_{t}$ for $t>b$, while $\partial P_{t}$ and $\operatorname{supp} f$ may have nonempty intersection for $a \leq t \leq b$. By (5), all the $c_{j}(t)$ are equal to zero for $t<a$ : the perturbation has not yet reached the vertex of the cone. The waves reflected from the vertex arise at the moment $t=a$. Generally speaking, the coefficients $c_{j}(t)$ do not vanish for $t>a$. For $a \leq t \leq b$, the smoothness of the functions $c_{j}$ depends on that of $f$. All the $c_{j}$ are infinitely differentiable for $t>b$.

Thus, the asymptotics (4) represents the hierarchy of the waves $c_{j} u_{j}$ reflected from the conical point. The "form" $u_{j}$ of a wave is a local element of the asymptotics (in the above sense), while the "intensity" $c_{j}(t)$ of the wave at a moment $t$ is determined by the evolution of the system in the past.

Presently, formulas similar to (4) and (5) for various situations (the Dirichlet and Neumann problems, homogeneous and nonhomogeneous boundary conditions, conical points and edges of positive dimensions, etc.) are indeed the basic results obtained in the topic in question. The strategy of the proofs is the same as in [1, where the wave operator $\partial_{t}^{2}-\Delta_{x}$ was considered with homogeneous Dirichlet boundary condition. 
This approach was developed in [2] for a certain class of hyperbolic systems in a wedge with spatial strongly elliptic part and nonhomogeneous Dirichlet boundary condition. The Lamé system falls into this class only under some additional restriction on the Lamé parameters (roughly speaking, only half of the usual parameter set is admitted). The results of the present paper imply that these additional restrictions can be lifted. Moreover, we give up the extra smoothness requirements imposed along the edge on the right-hand side of the problem in 1, 2. The Neumann problem for the Lamé system with homogeneous boundary condition in a domain with conical point was studied in 3 . In the present paper, in particular, we investigate the problem with nonhomogeneous condition in a domain having an edge of any dimension.

We discuss the method of proof. For this, we consider the problem

$$
\begin{array}{rlrl}
\mathcal{L}\left(x, D_{x}, D_{t}\right) u(x, t) & =f(x, t), & (x, t) & \in \mathbb{D} \times \mathbb{R}, \\
u(x, t) & =g(x, t), \quad & (x, t) \in \partial \mathbb{D} \times \mathbb{R},
\end{array}
$$

where $\mathbb{D}=\mathbb{K} \times \mathbb{R}^{m-n}$, and $\mathbb{K}$ is an open cone in $\mathbb{R}^{n}$ smooth outside the vertex; the vertex coincides with the origin. Suppose that the coefficients of $\mathcal{L}$ are constant off a compact set in $\overline{\mathbb{D}}$. Our approach splits into several stages: 1) energy estimates of solutions ("homogeneous" and "nonhomogeneous"); 2) a priori estimates of solutions in a scale of weighted spaces; 3 ) investigation of a model problem in $\mathbb{K} ; 4$ ) the asymptotics of solutions. The matter reduces to the study of a problem with complex parameter in the wedge,

$$
\begin{aligned}
\mathcal{L}\left(x, D_{x}, \tau\right) v(x) & =\varphi(x), & & x \in \mathbb{D}, \\
v(x) & =\psi(x), & & x \in \partial \mathbb{D},
\end{aligned}
$$

obtained by applying the Fourier transformation $\mathcal{F}_{t \rightarrow \tau}$ to (8) and (9); here $\tau=\sigma-i \gamma$, $\gamma=$ const $>0$, and $\sigma \in \mathbb{R}$.

We repeatedly use energy estimates to prove the existence of solutions and a priori estimates in weighted norms. In the homogeneous energy estimate

$$
\gamma^{2} \int_{\mathbb{D}}\left(\left|\nabla_{x} v(x)\right|^{2}+|\tau|^{2}|v(x)|^{2}\right) \mathrm{d} x \leq c \int_{\mathbb{D}}\left|\mathcal{L}\left(x, D_{x}, \tau\right) v(x)\right|^{2} \mathrm{~d} x,
$$

the function $v$ should vanish on $\partial \mathbb{D}$. A nonhomogeneous estimate is of the form

$$
\begin{aligned}
& \gamma^{2} \int_{\mathbb{D}}\left(\left|\nabla_{x} v(x)\right|^{2}+|\tau|^{2}|v(x)|^{2}\right) \mathrm{d} x+\gamma \int_{\partial \mathbb{D}}\left|\partial_{\nu} v(x)\right|^{2} \mathrm{~d} S \\
& \quad \leq c\left\{\int_{\mathbb{D}}\left|\mathcal{L}\left(x, D_{x}, \tau\right) v(x)\right|^{2} \mathrm{~d} x+\gamma \int_{\partial \mathbb{D}}\left(\left|\nabla_{s} v(x)\right|^{2}+|\tau|^{2}|v(x)|^{2}\right) \mathrm{d} S\right\},
\end{aligned}
$$

where $\nabla_{s}$ is the tangent gradient and $\partial_{\nu}$ the outward normal at $\partial \mathbb{D} \backslash\left(\mathcal{O} \times \mathbb{R}^{m-n}\right)$. Inequality (13) may involve a surface integral even in the case where $\left.v\right|_{\partial \mathbb{D}}=0$. The constants $c$ in (12) and (13) are independent of $v$ and $\tau=\sigma-i \gamma$, where $\sigma \in \mathbb{R}, \gamma \geq \gamma_{0}$, and $\gamma_{0}$ is sufficiently large.

We comment on inequality (13). We prove it only for the wedges $\mathbb{D}=\mathbb{K} \times \mathbb{R}^{m-n}$ such that $\partial \mathbb{K}$ can be given by an equation of the form $y_{n}=h\left(y_{1}, \ldots, y_{n-1}\right)$ in an appropriate coordinate system in $\mathbb{R}^{n}$. An example shows that, in general, this condition cannot be lifted. (Inequality (12) does not require such a restriction.) First, we verify the estimate for the functions smooth outside the edge and such that both sides in (13) are finite. Then, for such functions we introduce the operator

$$
\left\{v,\left.v\right|_{\partial \mathbb{D}},\left.\partial_{\nu} v\right|_{\partial \mathbb{D}}\right\} \mapsto\left\{\mathcal{L}\left(x, D_{x}, \tau\right) v,\left.v\right|_{\partial \mathbb{D}}\right\},
$$

which acts unboundedly from $\mathbf{H}:=H^{1}(\mathbb{D}) \times H^{1}(\partial \mathbb{D}) \times L_{2}(\partial \mathbb{D})$ to $L_{2}(\mathbb{D}) \times H^{1}(\partial \mathbb{D})$. The operator (14) admits closure, denoted by $\mathcal{A}(\tau)$. Estimate (13) extends to the domain 
$\mathcal{D}(\mathcal{A}(\tau))$. Any element $V=\left\{v,\left.v\right|_{\partial \mathbb{D}},\left.\partial_{\nu} v\right|_{\partial \mathbb{D}}\right\} \in \mathcal{D}(\mathcal{A}(\tau))$ is completely determined by its first component $v$. If

$$
\mathcal{A}(\tau) V=\{\varphi, \psi\} \in L_{2}(\mathbb{D}) \times H^{1}(\partial \mathbb{D}), \quad V \in \mathcal{D}(\mathcal{A}(\tau)),
$$

then $v$ is called a strong solution of problem (10), (11). The uniqueness of a strong solution follows from (13); the existence will be established in $\S 4$.

The solution of problem (10), (11) with $\psi=0$ can be linked with estimate (12), and this is the only way if the corresponding inequality (13) fails. If $\psi=0$ in (11) and both inequalities (12) and (13) are valid, then the solutions related to (12) and (13) coincide.

The entries of the deformation and stress tensors involve only first order derivatives of solutions. Therefore, the traces of these tensors on $\partial \mathbb{D}$ make sense for the strong solutions subject to (13). (Of course, we must return from a $\tau$-representation to a $t$ representation via the inverse Fourier transformation; it is important that the constant $c$ in (13) is independent of $\tau$.) If only estimate (12) is fulfilled, then the traces cannot be defined without some additional requirements on the right-hand side of the problem. The absence of inequality (13), say, for the Lamé system in the case of screens and cracks (corners of opening $2 \pi$ ) is a manifestation of the known "flaw" of the system: infinite stresses arise at the edge of a screen and at the tip of a crack.

For hyperbolic problems subject to the uniform Lopatinsky condition in domains with smooth boundary, estimates such as (13) were discussed by Agmon 7], Kreiss 8], Sakamoto [9], Agranovich [10, Volevich and Gindikin [11, and others (see the references in [11]). In such a situation, the matter reduces to the study of "local representatives," which are problems in the space $\mathbb{R}^{n}$ and in the half-space $\mathbb{R}_{+}^{n}$. Near an edge, such localization does not work. For this reason, to get inequality (13), we generalize the approach used by Gårding in 12 for a scalar second order hyperbolic equation with oblique derivative boundary condition in a domain with smooth boundary. (A detailed exposition was given by Hörmander in 13 for the problem with Dirichlet boundary condition.) For the same purpose, this trick was applied to the Lamé system in 2 under some additional restrictions on the Lamé coefficients; in the present paper we do not use these restrictions.

The next step consists in proving a priori weighted estimates of solutions that play a key role in the justification of asymptotic formulas. The estimates have a "zone" structure. In a neighborhood of the edge (at a distance no greater than const $/|\tau|$ from the edge), the estimate is "elliptic": the derivatives of the solution up to order 2 are majorized. Far from the edge, the estimate is "hyperbolic": the first order derivatives are majorized. Owing to such a zone structure, these inequalities turn out to be uniform with respect to the parameter $\tau$, i.e., the constants in the inequalities are independent of $\tau$. The proof is based on some known results in the theory of elliptic problems in domains with piecewise smooth boundary (see, e.g., 17]) and on the energy inequalities given in $\S \S 2$ and 3. The estimate discussed above will be called a mixed estimate.

The elliptic zone in a mixed estimate makes it possible to employ the machinery of the theory of elliptic boundary value problems when proving asymptotic formulas for solutions. In particular, this refers to the model problem in a cone $\mathbb{K}$, studied with the help of mixed estimates. The asymptotic formulas for solutions of such a problem are then employed to derive the asymptotics of solutions of the nonstationary problem in the wedge $\mathbb{D}=\mathbb{K} \times \mathbb{R}^{m-n}$ (with constant coefficients).

The paper is organized as follows. In $\S \S 2-5$ we study problems with a complex parameter that arise from time-dependent problems after the Fourier transformation in time. Homogeneous and nonhomogeneous energy estimates of solutions are given in $\S \S 2$ and 3, respectively. In $\S 4$ we introduce the strong solutions and prove their existence and uniqueness. Weighted a priori estimates are presented in $\S 5$. The boundary value 
problem in a cone is studied in $\S 6$ in a scale of weighted space. Asymptotic formulas for solutions of the nonstationary problem in a wedge are collected in $\S 7$. Then, in $\S \S 8,9$, we prove energy and mixed estimates for the Dirichlet and Neumann problems in a bounded domain $G$ with edges. The final stage - derivation of asymptotic formulas in the general case - is not presented in the paper. In essence, that stage consists in compiling some results of the theory of elliptic problems. In particular, the asymptotics of solutions of time-dependent problems in domains with conical points can be obtained by a simple modification of the argument in $\S 7$ of the present paper; some examples of this kind were given in [1, 3, 29]. The information on elliptic theory needed in the case of edges of positive dimension can be found, e.g., in [17.

We indicate some publications related to hyperbolic problems in nonsmooth domains. The wave equation was considered in [18] (a wedge with edge of codimension 2, an explicit formula for solutions), in [19, 20] (domains with edges, the fundamental solutions, propagation of singularities; the approach was based on the functional calculus for the Laplace operator), in [21, 22] (microlocal analysis), and in 23] (the homogeneous Dirichlet boundary condition); see also the references in those papers. The monographs [24]-[26] were devoted to some model situations and were built mostly on explicit formulas. Some general approach to the study of asymptotics was suggested in [27, 28] (strongly hyperbolic systems in a domain with conical points and homogeneous Dirichlet condition); this approach does not lead to formulas for the coefficients. Neither methods nor results of the publications mentioned in this paragraph were used in the present paper. In conclusion, we note that the method of [1] was developed in 29] for the time-dependent Maxwell system in domains with edges.

\section{§2. Homogeneous energy estimates for solutions of Boundary value PROBLEMS WITH PARAMETER IN A WEDGE}

Let $\mathbb{K}$ be an open cone in the space $\mathbb{R}^{n}$ with vertex $\mathcal{O}$ at the origin, and let $\Omega=$ $\mathbb{K} \cap \mathcal{S}^{n-1}$ be a domain lying on the sphere $\mathcal{S}^{n-1}$ and having smooth $(n-2)$-dimensional boundary $\partial \Omega$. We denote by $\mathbb{D}$ the $m$-dimensional wedge $\mathbb{K} \times \mathbb{R}^{m-n}$. In the cylinder $\mathcal{Q}=\{(x, t): x \in \mathbb{D}, t \in \mathbb{R}\}$, we consider the system of equations

$$
\begin{array}{r}
\left(\mathcal{L}\left(x, D_{x}, D_{t}\right) u\right)_{p}=\partial_{t}^{2} u_{p}(x, t)-\partial_{k}\left(a_{p q}^{k l}(x) \partial_{l} u_{q}(x, t)\right)=f_{p}(x, t), \\
p=1, \ldots, m, \quad(x, t) \in \mathbb{D} \times \mathbb{R},
\end{array}
$$

where $\partial_{k}=\partial / \partial x_{k}, D_{x_{k}}=-i \partial_{k}$, etc., and where summation is meant over $q, k, l=$ $1, \ldots, m$. The coefficients $a_{p q}^{k l}$ are real functions of class $\mathcal{C}^{\infty}(\overline{\mathbb{D}})$ that are constant off a compact set in $\mathbb{R}^{m}$. Also, let

$$
a_{p q}^{k l}=a_{q p}^{l k}, \quad a_{p q}^{k l}=a_{k q}^{p l}
$$

Moreover, we assume that

$$
\sum a_{p q}^{k l}(x) \xi_{k}^{p} \xi_{l}^{q} \geq c \sum\left(\xi_{k}^{p}\right)^{2}
$$

where $\xi_{k}^{p}=\xi_{p}^{k}, \xi_{p}^{k}$ are real numbers, $c$ is a positive constant, and summation is over $k, l, p, q=1, \ldots, m$. We supplement (15) with the Dirichlet boundary condition

$$
u_{p}=g_{p} \quad \text { on }(\partial \mathbb{D} \backslash M) \times \mathbb{R}, p=1, \ldots, m,
$$

or with the Neumann condition

$$
\left(\mathcal{B}\left(x, \partial_{x}\right) u\right)_{p}=\nu_{k}(x) a_{p q}^{k l}(x) \partial_{l} u_{q}=g_{p} \quad \text { on }(\partial \mathbb{D} \backslash M) \times \mathbb{R}, p=1, \ldots, m,
$$

where $\nu=\left(\nu_{1}, \ldots, \nu_{n}\right)$ is the outward unit normal to $\partial \mathbb{D}$ and $M=\{\mathcal{O}\} \times \mathbb{R}^{m-n}$ is the edge of the wedge. 
In the present paper we use various estimates similar to the Korn inequality. They are taken from [6]. In some cases we slightly modify such inequalities for our goals. We give the corresponding formulations and explanations when needed. The next lemma contains a result of this kind for a wedge.

Lemma 2.0.1. For vector-valued functions $u=\left(u_{1}, \ldots, u_{m}\right)$ in the Sobolev space $H^{1}(\mathbb{D})$, we have

$$
\int_{\mathbb{D}} \sum_{i}\left|\nabla u_{i}(x)\right|^{2} \mathrm{~d} x \leq c \int_{\mathbb{D}} \sum_{i, k}\left|\partial_{k} u_{i}(x)+\partial_{i} u_{k}(x)\right|^{2} \mathrm{~d} x
$$

Proof. The inequality

$$
\int_{\mathbb{D}}|\nabla(u(x)-A x)|^{2} \mathrm{~d} x \leq c \int_{\mathbb{D}} \sum_{i, k}\left|\partial_{k} u_{i}(x)+\partial_{i} u_{k}(x)\right|^{2} \mathrm{~d} x
$$

for a wedge with Lipschitz boundary was proved in [6] under the assumptions that $u \in$ $H^{1}(G)$ for any bounded subdomain $G$ of the wedge $\mathbb{D}$, the right-hand side is finite, and $A$ is a certain skew-symmetric constant matrix (see [6, §3]). In our case, the boundary $\partial \mathbb{D}$ can be non-Lipschitz (for instance, if $n=2$ and the opening of the wedge equals $2 \pi)$. We split the cone $\mathbb{K}$ into finitely many cones $\mathbb{K}_{\mu}$ with Lipschitz boundary. Then the wedge $\mathbb{D}$ turns out to be split into the Lipschitz parts $\mathbb{D}_{\mu}=\mathbb{K}_{\mu} \times \mathbb{R}^{m-n}$ (say, if $n=2$ and the opening equals $2 \pi$, then the wedge $\mathbb{D}$ should be split into two half-spaces). Since $u \in H^{1}\left(\mathbb{D}_{\mu}\right)$, inequality (21) is true for every wedge $\mathbb{D}_{\mu}$, with $A=0$. Adding such estimates, we arrive at (20).

We start with the Dirichlet problem

$$
\begin{aligned}
\mathcal{L}\left(x, D_{x}, D_{t}\right) u(x, t) & =f(x, t), & & (x, t) \in \mathbb{D} \times \mathbb{R}, \\
u(x, t) & =0, & & (x, t) \in(\partial \mathbb{D} \backslash M) \times \mathbb{R} .
\end{aligned}
$$

Applying the Fourier transformation $\mathcal{F}_{t \rightarrow \tau}$ to (22), (23) and putting

$$
\widehat{u}(x, \tau)=\mathcal{F}_{t \rightarrow \tau} u(x, t)=\frac{1}{\sqrt{2 \pi}} \int_{-\infty}^{+\infty} e^{-i \tau t} u(x, t) \mathrm{d} t, \quad \tau=\sigma-i \gamma, \sigma \in \mathbb{R}, \quad \gamma>0,
$$

we obtain the following problem in the wedge $\mathbb{D}$ with parameter $\tau$ :

$$
\begin{aligned}
\mathcal{L}\left(x, D_{x}, \tau\right) \widehat{u}(x, \tau) & =\widehat{f}(x, \tau), & & x \in \mathbb{D}, \\
\widehat{u}(x, \tau) & =0, & & x \in \partial \mathbb{D} \backslash M .
\end{aligned}
$$

We introduce the linear space $\mathfrak{P}$ of functions on $\mathbb{D}$ such that

$$
v \in C^{\infty}(\overline{\mathbb{D}} \backslash M) \cap H^{1}(\mathbb{D}),\left.\quad v\right|_{\partial \mathbb{D}}=0, \quad \mathcal{L}\left(x, D_{x}, 0\right) v \in L_{2}(\mathbb{D}) ;
$$

moreover, we assume that

$$
\int_{\mathbb{D}}\langle A v, w\rangle \mathrm{d} x=\int_{\mathbb{D}}\left\langle A^{k l} \partial_{k} v, \partial_{l} w\right\rangle \mathrm{d} x
$$

for any $v$ and $w$ in $\mathfrak{P}$, where $A\left(x, D_{x}\right)=D_{k} A^{k l}(x) D_{l}, A^{k l}(x)=\left\|a_{p q}^{k l}(x)\right\|_{p, q=1}^{m}$, and $\langle\cdot, \cdot\rangle$ denotes the inner product in $\mathbb{C}^{m}$.

We comment on these requirements. In what follows we use the fact that the weak solutions of problem (24), (25) with fixed $\tau$ and $\widehat{f} \in C_{c}^{\infty}(\overline{\mathbb{D}} \backslash M)$ are in $\mathfrak{P}$ (such solutions are defined in $\S 4$ ). The only thing that must be checked in (26) is the relation $v \in$ 
$C^{\infty}(\overline{\mathbb{D}} \backslash M)$; this is a usual property of solutions of elliptic problems. We explain how to verify (27). Assuming that $0<\varepsilon<R<+\infty$, we have

$$
\begin{aligned}
\int_{\mathbb{D}} \cap\{\varepsilon<r<R\} & \langle A v, w\rangle \mathrm{d} x \\
\quad= & \int_{\mathbb{D} \cap\{\varepsilon<r<R\}}\left\langle A^{k l} \partial_{k} v, \partial_{l} w\right\rangle \mathrm{d} x-i\left(\int_{\mathbb{D} \cap\{r=\varepsilon\}}+\int_{\mathbb{D} \cap\{r=R\}}\right)\left\langle A^{k l} \partial_{k} v, w\right\rangle \nu_{l} \mathrm{~d} S,
\end{aligned}
$$

where $x=(y, z), y=\left(x_{1}, \ldots, x_{n}\right), z=\left(x_{n+1}, \ldots, x_{m}\right), r=|y|$ is the distance from $x$ to the edge, and $\nu_{l}(x)$ is the outward unit normal. Since the function $x \mapsto\left\langle A^{k l}(x) \partial_{k} v(x), w(x)\right\rangle$ is integrable over $\mathbb{D}$, there exists a sequence $\{R(q)\}_{q=1}^{\infty}, R(q) \rightarrow+\infty$, such that

$$
\lim _{q \rightarrow \infty} \int_{\mathbb{D} \cap\{r=R(q)\}}\left|\left\langle A^{k l} \partial_{k} v, w\right\rangle\right| \mathrm{d} S=0 .
$$

We put $r=R(q)$ in (28). Then the corresponding surface integral vanishes as $q \rightarrow \infty$. To clarify the behavior of the other surface integral as $\varepsilon \rightarrow 0$, we can use the asymptotics of the solution of (24), (25) in a neighborhood of the edge. Assume, for instance, that $n=m, \mathbb{D}=\mathbb{K}$, and the "edge" is a conical point. By a known asymptotic formula, if $\widehat{f} \in C_{c}^{\infty}(\overline{\mathbb{D}} \backslash M)$ and $v \in H^{1}(\mathbb{D})$, then $D^{\alpha} v(x)=O\left(r^{\mu-|\alpha|}\right)$ as $r \rightarrow 0$, where $|\alpha|=0,1$ and $\mu$ is some number satisfying $\mu>(2-n) / 2$. (Here by $v$ we mean the solution of (24), (25).) Therefore, the integral over $\mathbb{D} \cap\{r=\epsilon\}$ in (28) tends to zero as $\epsilon \rightarrow 0$. We restrict ourselves to the above remarks. Some results on the asymptotics of solutions of elliptic problems near edges will be recalled in $\S \S 5$ and 6 . A detailed description of the asymptotics can be found, e.g., in [17.

Proposition 2.0.2. For the functions $v$ in $\mathfrak{P}$, we have

$$
\gamma^{2} \int_{\mathbb{D}}\left(|\tau|^{2}|v(x)|^{2}+\left|\nabla_{x} v(x)\right|^{2}\right) \mathrm{d} x \leq c \int_{\mathbb{D}}\left|\mathcal{L}\left(x, D_{x}, \tau\right) v(x)\right|^{2} \mathrm{~d} x
$$

with constant $c$ depending neither on $v$, nor on the parameter $\tau=\sigma-i \gamma$, where $\sigma \in \mathbb{R}$ and $\gamma>0$.

Proof. We write $\mathcal{L}$ in the form

$$
\mathcal{L}\left(x, D_{x}, D_{t}\right) u(x, t)=\partial_{t}^{2} u(x, t)+A\left(x, D_{x}\right) u(x, t) .
$$

Using (22) and (30), we obtain

$$
\int_{\mathbb{D}} \int_{-\infty}^{t}\left(\left\langle\partial_{s}^{2} u, \partial_{s} u\right\rangle+\left\langle A u, \partial_{s} u\right\rangle\right) \mathrm{d} x \mathrm{~d} s=\int_{\mathbb{D}} \int_{-\infty}^{t}\left\langle f, \partial_{s} u\right\rangle \mathrm{d} x \mathrm{~d} s
$$

here $u(x, t)=\chi(t) v(x), v \in \mathfrak{P}$, and the two functions $t \mapsto \chi(t)$ and $t \mapsto e^{-\gamma t} \chi(t)$ are in the Schwartz class $\mathcal{S}(\mathbb{R})$. Adding identity (31) and the adjoint one, we see that

$$
\begin{gathered}
\int_{\mathbb{D}} \int_{-\infty}^{t}\left(\partial_{s}\left|\partial_{s} u\right|^{2}+\left\langle A\left(x, D_{x}\right) u, \partial_{s} u\right\rangle+\left\langle\partial_{s} u, A\left(x, D_{x}\right) u\right\rangle\right) \mathrm{d} x \mathrm{~d} s \\
=2 \operatorname{Re} \int_{\mathbb{D}} \int_{-\infty}^{t}\left\langle f, \partial_{s} u\right\rangle \mathrm{d} x \mathrm{~d} s .
\end{gathered}
$$

Since, by (27),

$$
\int_{\mathbb{D}}\langle A v, w\rangle \mathrm{d} x=\int_{\mathbb{D}} a_{i j}^{k l} \partial_{k} v_{i} \overline{\partial_{l} w_{j}} \mathrm{~d} x
$$

for $v, w$ in $\mathfrak{P}$, we have

$$
\left(A u, \partial_{t} u\right)_{\mathbb{D}}+\left(\partial_{t} u, A u\right)_{\mathbb{D}}=\partial_{t}(A u, u)_{\mathbb{D}}
$$


Combined with (32), this leads to the formula

$$
\left\|\partial_{t} u(\cdot, t) ; L_{2}(\mathbb{D})\right\|^{2}+(A u(\cdot, t), u(\cdot, t))_{\mathbb{D}}=2 \operatorname{Re} \int_{\mathbb{D}} \int_{-\infty}^{t}\left\langle f, \partial_{s} u\right\rangle \mathrm{d} x \mathrm{~d} s .
$$

Applying (33) once again and recalling that the tensor $\left\{a_{i j}^{k l}\right\}$ is symmetric, we obtain

$$
\begin{aligned}
(A u(\cdot, t), u(\cdot, t))_{\mathbb{D}} & =\int_{\mathbb{D}} a_{i j}^{k l}(x) \partial_{k} u_{i}(x, t) \overline{\partial_{l} u_{j}(x, t)} \mathrm{d} x \\
& \geq c \int_{\mathbb{D}} \sum_{i, k}\left|\partial_{k} u_{i}(x, t)+\partial_{i} u_{k}(x, t)\right|^{2} \mathrm{~d} x .
\end{aligned}
$$

Here we have used the inequality

$$
\sum a_{i j}^{k l}(x) \eta_{i}^{k} \overline{\eta_{j}^{l}} \geq c \sum\left|\eta_{i}^{k}\right|^{2}
$$

where the complex numbers $\eta_{i}^{k}$ satisfy $\eta_{i}^{k}=\eta_{k}^{i}, i, k=1, \ldots, m$. (To get (136), it suffices to separate the real and imaginary parts, take (16) into account, and apply (17).) Now (34) implies the estimate

$$
\begin{gathered}
\left\|\partial_{t} u(\cdot, t) ; L_{2}(\mathbb{D})\right\|^{2}+\int_{\mathbb{D}} \sum_{i, k}\left|\partial_{k} u_{i}(x, t)+\partial_{i} u_{k}(x, t)\right|^{2} \mathrm{~d} x \\
\leq c \int_{-\infty}^{t}\left\|f(\cdot, s) ; L_{2}(\mathbb{D})\right\| \cdot\left\|\partial_{s} u(\cdot, s) ; L_{2}(\mathbb{D})\right\| \mathrm{d} s .
\end{gathered}
$$

Therefore, by (20),

$$
\left\|\nabla_{x, t} u(\cdot, t) ; L_{2}(\mathbb{D})\right\|^{2} \leq c \int_{-\infty}^{t}\left\|f(\cdot, s) ; L_{2}(\mathbb{D})\right\| \cdot\left\|\partial_{s} u(\cdot, s) ; L_{2}(\mathbb{D})\right\| \mathrm{d} s .
$$

We denote the integrand on the right by $h(t)$, multiply this inequality by $e^{-2 \gamma t}$, and integrate, obtaining

$$
\begin{aligned}
\int_{-\infty}^{+\infty} & e^{-2 \gamma t}\left\|\nabla_{x, t} u(\cdot, t) ; L_{2}(\mathbb{D})\right\|^{2} \mathrm{~d} t \leq c \int_{-\infty}^{+\infty} e^{-2 \gamma t} \mathrm{~d} t \int_{-\infty}^{t} h(s) \mathrm{d} s \\
& =\int_{-\infty}^{+\infty} h(s) \mathrm{d} s \int_{s}^{+\infty} e^{-2 \gamma t} \mathrm{~d} t=\frac{c}{2 \gamma} \int_{-\infty}^{+\infty} e^{-2 \gamma t} h(t) \mathrm{d} t \\
& \leq \frac{c}{2 \gamma}\left(\int_{-\infty}^{+\infty} e^{-2 \gamma t}\left\|f(\cdot, t) ; L_{2}(\mathbb{D})\right\|^{2} \mathrm{~d} t\right)^{1 / 2}\left(\int_{-\infty}^{+\infty} e^{-2 \gamma t}\left\|\partial_{t} u(\cdot, t) ; L_{2}(\mathbb{D})\right\|^{2} \mathrm{~d} t\right)^{1 / 2} .
\end{aligned}
$$

Consequently,

$$
\gamma^{2} \int_{-\infty}^{+\infty} e^{-2 \gamma t}\left\|\nabla_{x, t} u(\cdot, t) ; L_{2}(\mathbb{D})\right\|^{2} \mathrm{~d} t \leq c \int_{-\infty}^{+\infty} e^{-2 \gamma t}\left\|f(\cdot, t) ; L_{2}(\mathbb{D})\right\|^{2} \mathrm{~d} t .
$$

We put $u(x, t)=\chi(t) v(x)$ and apply the Parseval identity for the Fourier transformation $\mathcal{F}_{t \rightarrow \tau}$. We have

$$
\begin{gathered}
\gamma^{2} \int_{\mathbb{D}} \mathrm{d} x \int_{-\infty}^{+\infty}|\widehat{\chi}(\sigma-i \gamma)|^{2}\left(\left|\nabla_{x} v(x)\right|^{2}+|\tau|^{2}|v(x)|^{2}\right) \mathrm{d} \sigma \\
\leq c \int_{\mathbb{D}} \mathrm{d} x \int_{-\infty}^{+\infty}|\widehat{\chi}(\sigma-i \gamma)|^{2}\left|\mathcal{L}\left(x, D_{x}, \tau\right) v(x)\right|^{2} \mathrm{~d} \sigma .
\end{gathered}
$$

Since the function $\chi$ can be chosen arbitrarily, this implies (29). 
We turn to the Neumann problem

$$
\begin{aligned}
\mathcal{L}\left(x, D_{x}, D_{t}\right) u(x, t) & =f(x, t), & & (x, t) \in \mathbb{D} \times \mathbb{R}, \\
\mathcal{B}\left(x, D_{x}\right) u(x, t) & =0, & & (x, t) \in(\partial \mathbb{D} \backslash M) \times \mathbb{R},
\end{aligned}
$$

where $\mathcal{B}\left(x, D_{x}\right)=\nu_{k}(x) a_{i j}^{k l}(x) D_{l}$. Applying the Fourier transformation $\mathcal{F}_{t \rightarrow \tau}$, we obtain a problem with parameter $\tau$ :

$$
\begin{aligned}
\mathcal{L}\left(x, D_{x}, \tau\right) \widehat{u}(x, \tau) & =\widehat{f}(x, \tau), & & x \in \mathbb{D}, \\
\mathcal{B}\left(x, D_{x}\right) \widehat{u}(x, \tau) & =0, & & x \in \partial \mathbb{D} \backslash M .
\end{aligned}
$$

Proposition 2.0.3. For the elements of the set

$$
\left\{v \in \mathcal{C}^{\infty}(\overline{\mathbb{D}} \backslash M) \cap H^{1}(\mathbb{D}):\left.\mathcal{B}\left(x, D_{x}\right) v\right|_{\partial \mathbb{D} \backslash M}=0, \mathcal{L}\left(x, D_{x}, 0\right) v \in L_{2}(\mathbb{D})\right\},
$$

inequality (29) is fulfilled with constant $c$ depending neither on $v$ nor on $\tau=\sigma-i \gamma$ $(\sigma \in \mathbb{R}, \gamma>0)$.

The proof is much the same as that of Proposition 2.0.2 (with evident changes).

\section{§3. Nonhomogeneous EnERgy estimates for SOlutions of BOUNDARY VALUE PROBLEMS WITH PARAMETER IN A WEDGE}

We consider system (15) in the cylinder $\mathbb{D} \times \mathbb{R}$ with boundary conditions (18) or (19). In what follows, it is convenient to write $x_{m+1}$ instead of $t$. We also put

$$
g_{i j}^{\alpha \beta}= \begin{cases}-a_{i j}^{\alpha \beta}, & 1 \leq \alpha, \beta \leq m, \\ \delta_{i j}, & \alpha=\beta=m+1,\end{cases}
$$

assuming that $g_{i j}^{\alpha \beta}=0$ for all the other indices $i, j, \alpha, \beta$; the Greek indices range over $1, \ldots, m+1$, while, as before, the Latin indices range over $1, \ldots, m$. Then the operators $\mathcal{L}, \mathcal{B}$ can be rewritten in the form

$$
\begin{aligned}
\left(\mathcal{L}\left(x, D_{x}, D_{t}\right) u\right)_{i} & =\partial_{\alpha}\left(g_{i j}^{\alpha \beta} \partial_{\beta} u_{j}\right), \\
\mathcal{B}\left(x, \partial_{x}\right) & =-\nu_{\alpha} g_{i j}^{\alpha \beta} \partial_{\beta} u_{j} .
\end{aligned}
$$

The first of relations (16) means that

$$
g_{i j}^{\alpha \beta}=g_{j i}^{\beta \alpha}, \quad i, j=1, \ldots, m, \quad \alpha, \beta=1, \ldots, m+1 .
$$

When proving estimates for solutions, we make use of the following statement.

Lemma 3.0.4. Let $\left\{f^{\alpha}\right\}_{\alpha=1}^{m+1}$ be a collection of real functions of class $\mathcal{C}^{1}(\overline{\mathbb{D}})$. Then, on the set $(\overline{\mathbb{D}} \backslash M) \times \mathbb{R}$, for the functions in $C^{2}((\overline{\mathbb{D}} \backslash M) \times \mathbb{R})$ we have the identity

$$
\begin{aligned}
2 \operatorname{Re} & \left(e^{-2 \gamma t} f^{\sigma} \overline{\partial_{\sigma} u_{i}} \partial_{\alpha}\left(g_{i j}^{\alpha \beta} \partial_{\beta} u_{j}\right)\right) \\
= & e^{-2 \gamma t}\left(\partial_{\sigma} f^{\sigma}\right) g_{i j}^{\alpha \beta} \partial_{\alpha} u_{i} \overline{\partial_{\beta} u_{j}} \\
& +\operatorname{Re} \partial_{\alpha}\left(e^{-2 \gamma t}\left\{2 f^{\sigma} \overline{\partial_{\sigma} u_{i}} g_{i j}^{\alpha \beta} \partial_{\beta} u_{j}-f^{\alpha} g_{i j}^{\sigma \beta} \partial_{\sigma} u_{i} \overline{\partial_{\beta} u_{j}}\right\}\right) \\
& +2 \gamma e^{-2 \gamma t} \operatorname{Re}\left(2 f^{\sigma} \overline{\partial_{\sigma} u_{i}} \partial_{t} u_{i}-f^{m+1} g_{i j}^{\alpha \beta} \partial_{\alpha} u_{i} \overline{\partial_{\beta} u_{j}}\right) \\
& +e^{-2 \gamma t} f^{\sigma}\left(\partial_{\sigma} g_{i j}^{\alpha \beta}\right) \partial_{\alpha} u_{i} \overline{\partial_{\beta} u_{j}}-2 \operatorname{Re}\left(e^{-2 \gamma t}\left(\partial_{\alpha} f^{\sigma}\right) \overline{\partial_{\sigma} u_{i}} g_{i j}^{\alpha \beta} \partial_{\beta} u_{j}\right) .
\end{aligned}
$$


Proof. We have

$$
\begin{aligned}
\partial_{\alpha} & \left(f^{\sigma} \overline{\partial_{\sigma} u_{i}} g_{i j}^{\alpha \beta} \partial_{\beta} u_{j}\right) \\
& =f^{\sigma} \overline{\partial_{\sigma} u_{i}} \partial_{\alpha}\left(g_{i j}^{\alpha \beta} \partial_{\beta} u_{j}\right)+f^{\sigma} \overline{\partial_{\alpha} \partial_{\sigma} u_{i}} g_{i j}^{\alpha \beta} \partial_{\beta} u_{j}+\left(\partial_{\alpha} f^{\sigma}\right) \overline{\partial_{\sigma} u_{i}} g_{i j}^{\alpha \beta} \partial_{\beta} u_{j}
\end{aligned}
$$

and

$$
\begin{aligned}
& f^{\sigma} \partial_{\sigma}\left(\partial_{\alpha} u_{i} g_{i j}^{\alpha \beta} \overline{\partial_{\beta} u_{j}}\right) \\
& \quad=f^{\sigma} \partial_{\alpha} u_{i}\left(\partial_{\sigma} g_{i j}^{\alpha \beta}\right) \overline{\partial_{\beta} u_{j}}+f^{\sigma} \partial_{\alpha} \partial_{\sigma} u_{i} g_{i j}^{\alpha \beta} \overline{\partial_{\beta} u_{j}}+f^{\sigma} \partial_{\alpha} u_{i} g_{i j}^{\alpha \beta} \overline{\partial_{\sigma} \partial_{\beta} u_{j}} .
\end{aligned}
$$

As before, repetition of indices implies summation. By (45), the last relation can be written as

$$
f^{\sigma} \partial_{\sigma}\left(\partial_{\alpha} u_{i} g_{i j}^{\alpha \beta} \overline{\partial_{\beta} u_{j}}\right)=f^{\sigma} \partial_{\alpha} u_{i}\left(\partial_{\sigma} g_{i j}^{\alpha \beta}\right) \overline{\partial_{\beta} u_{j}}+2 \operatorname{Re}\left(f^{\sigma} \partial_{\alpha} \partial_{\sigma} u_{i} g_{i j}^{\alpha \beta} \overline{\partial_{\beta} u_{j}}\right) .
$$

We substitute the expression for $\operatorname{Re}\left(f^{\sigma} \partial_{\alpha} \partial_{\sigma} u_{i} g_{i j}^{\alpha \beta} \overline{\partial_{\beta} u_{j}}\right)$ obtained from (48) into the real part of (47). This yields

$$
\begin{aligned}
2 \operatorname{Re} & \left(\partial_{\alpha}\left(f^{\sigma} \overline{\partial_{\sigma} u_{i}} g_{i j}^{\alpha \beta} \partial_{\beta} u_{j}\right)\right) \\
= & 2 \operatorname{Re}\left(f^{\sigma} \overline{\partial_{\sigma} u_{i}} \partial_{\alpha}\left(g_{i j}^{\alpha \beta} \partial_{\beta} u_{j}\right)\right)+f^{\sigma} \partial_{\sigma}\left(\partial_{\alpha} u_{i} g_{i j}^{\alpha \beta} \overline{\partial_{\beta} u_{j}}\right) \\
& -f^{\sigma} \partial_{\alpha} u_{i}\left(\partial_{\sigma} g_{i j}^{\alpha \beta}\right) \overline{\partial_{\beta} u_{j}}+2 \operatorname{Re}\left(\partial_{\alpha} f^{\sigma}\right) \overline{\partial_{\sigma} u_{i}} g_{i j}^{\alpha \beta} \partial_{\beta} u_{j} .
\end{aligned}
$$

We reshape the second term on the right:

$$
\begin{aligned}
& f^{\sigma} \partial_{\sigma}\left(g_{i j}^{\alpha \beta} \partial_{\alpha} u_{i} \overline{\partial_{\beta} u_{j}}\right)=\partial_{\sigma}\left(f^{\sigma} g_{i j}^{\alpha \beta} \partial_{\alpha} u_{i} \overline{\partial_{\beta} u_{j}}\right)-\left(\partial_{\sigma} f^{\sigma}\right) g_{i j}^{\alpha \beta} \partial_{\alpha} u_{i} \overline{\partial_{\beta} u_{j}} \\
& \quad=\partial_{\alpha}\left(f^{\alpha} g_{i j}^{\sigma \beta} \partial_{\sigma} u_{i} \overline{\partial_{\beta} u_{j}}\right)-\left(\partial_{\sigma} f^{\sigma}\right) g_{i j}^{\alpha \beta} \partial_{\alpha} u_{i} \overline{\partial_{\beta} u_{j}} .
\end{aligned}
$$

Identity (49) means that

$$
\begin{aligned}
2 \operatorname{Re}\left(f^{\sigma} \overline{\partial_{\sigma} u_{i}} \partial_{\alpha}\left(g_{i j}^{\alpha \beta} \partial_{\beta} u_{j}\right)\right)= & \operatorname{Re}\left(\partial_{\alpha}\left\{2 f^{\sigma} \overline{\partial_{\sigma} u_{i}} g_{i j}^{\alpha \beta} \partial_{\beta} u_{j}-f^{\alpha} g_{i j}^{\sigma \beta} \partial_{\sigma} u_{i} \overline{\partial_{\beta} u_{j}}\right\}\right) \\
& +f^{\sigma}\left(\partial_{\sigma} g_{i j}^{\alpha \beta}\right) \partial_{\alpha} u_{i} \overline{\partial_{\beta} u_{j}}+\left(\partial_{\sigma} f^{\sigma}\right) g_{i j}^{\alpha \beta} \partial_{\alpha} u_{i} \overline{\partial_{\beta} u_{j}} \\
& -2 \operatorname{Re}\left(\left(\partial_{\alpha} f^{\sigma}\right) \overline{\partial_{\sigma} u_{i}} g_{i j}^{\alpha \beta} \partial_{\beta} u_{j}\right) .
\end{aligned}
$$

It remains to multiply by $e^{-\gamma t}$ and bring the exponential under the derivation sign.

3.1. Estimates of solutions with Dirichlet boundary condition. Here we prove energy estimates for solutions in an admissible wedge in the sense of the following definition.

Definition 3.1.1. The wedge $\mathbb{D}=\mathbb{K} \times \mathbb{R}^{m-n} \subset \mathbb{R}^{m}$ is said to be admissible if the boundary $\partial \mathbb{K}$ of the cone can be given by an equation of the form $y_{n}=\psi\left(y_{1}, \ldots, y_{n-1}\right)$ (possibly after a rotation of the coordinate system $\left(y_{1}, \ldots, y_{n}\right)$ in $\left.\mathbb{R}^{n}\right)$; the function $\psi$ is assumed to be smooth for $\left|y_{1}\right|+\cdots+\left|y_{n-1}\right|>0$.

If a wedge $\mathbb{D}$ is admissible, then there exists a vector $l=\left(l_{1}, \ldots, l_{n}\right)$ such that

$$
\langle l, \nu(y)\rangle \geq c=\text { const }>0
$$

for all $y \in \partial \mathbb{K} \backslash \mathcal{O}$, where $\nu=\left(\nu_{1}, \ldots, \nu_{n}\right)$ is the field of outward unit normals to $\partial \mathbb{K} \backslash \mathcal{O}$. (It can be shown that the existence of such a vector $l$ is equivalent to the admissibility of the wedge in the sense of Definition 3.1.1.)

Let $H^{1}(\partial \mathbb{D})$ denote the completion of the traces on $\partial \mathbb{D}$ of the functions in $C_{c}^{\infty}(\overline{\mathbb{D}})$ with respect to the norm

$$
\left(\int_{\partial \mathbb{D}}\left(|u|^{2}+\left|\partial_{z} u\right|^{2}+\left|\partial_{r} u\right|^{2}+\frac{1}{r^{2}}\left|\nabla_{\omega} u\right|^{2}\right) \mathrm{d} S\right)^{1 / 2},
$$


where $(r, \omega, z)$ are the cylindrical coordinates of $x=(y, z)$, while $r=|y|$ and $\omega$ are the (local) coordinates of the point $y /|y|$ on $S^{n-1}$.

We denote by $\mathfrak{M}$ the set of functions in $C^{2}(\overline{\mathbb{D}} \backslash M)$ that satisfy

$$
v(x)=C+O\left(r^{(3-n) / 2+\varepsilon}\right), \quad D v(x)=O\left(r^{(3-n) / 2+\varepsilon-1}\right)
$$

as $r \rightarrow 0$, where $n \leq 3, C$ is a constant, and $\varepsilon$ is a positive number ( $C$ and $\varepsilon$ may depend on $v$ ); for $n>3$ we put $C=0$. Moreover,

$$
v \in H^{1}(\mathbb{D}), \quad \mathcal{L}\left(x, D_{x}, \tau\right) v \in L_{2}(\mathbb{D}),\left.\quad v\right|_{\partial \mathbb{D}} \in H^{1}(\partial \mathbb{D}) .
$$

Proposition 3.1.2. If $\mathbb{D}$ is an admissible wedge, then for the elements of $\mathfrak{M}$ we have

$$
\begin{aligned}
& \gamma^{2} \int_{\mathbb{D}}\left(\left|\nabla_{x} v(x)\right|^{2}+|\tau|^{2}|v(x)|^{2}\right) \mathrm{d} x+\gamma \int_{\partial \mathbb{D}}\left|\partial_{\nu} v\right|^{2} \mathrm{~d} S \\
& \quad \leq c\left\{\int_{\mathbb{D}}\left|\mathcal{L}\left(x, D_{x}, \tau\right) v(x)\right|^{2} \mathrm{~d} x+\gamma \int_{\partial \mathbb{D}}\left(|\tau|^{2}|v(x)|^{2}+\left|\nabla_{s} v(x)\right|^{2}\right) \mathrm{d} S\right\},
\end{aligned}
$$

where $\nabla_{s}$ denotes the tangent gradient on $\partial \mathbb{D} \backslash \mathcal{O}$, the constant $c$ is independent of $v$ and of $\tau=\sigma-i \gamma$ with $\sigma \in \mathbb{R}$ and $\gamma \geq \gamma_{0}$; here $\gamma_{0}$ is a sufficiently large positive number.

Remark 3.1.3. Conditions (52) are fulfilled for the (weak) solutions of the Dirichlet problem

$$
\begin{aligned}
\mathcal{L}\left(x, D_{x}, \tau\right) v(x) & =f(x), & & x \in \mathbb{D}, \\
v(x) & =g(x), & & x \in \partial \mathbb{D},
\end{aligned}
$$

with $f \in C_{c}^{\infty}(\overline{\mathbb{D}} \backslash M)$ and $g \in C_{c}^{\infty}(\partial \mathbb{D})$. (Such solutions will be defined in $\S 4$.) If the wedge is admissible, then the results in [30] imply that these solutions also satisfy (51) with $C=0$. The following example shows that the admissibility requirement in Proposition 3.1.2 cannot be dropped.

Let $m=n=2$, and let $\mathbb{D}=\mathbb{K}=\{(r, \varphi):-\pi<\varphi<\pi, 0<r<+\infty\}$. Consider the Dirichlet problem for the Lamé system

$$
\begin{array}{rlrl}
\triangle u+\frac{1}{1-2 \varkappa} \nabla \operatorname{div} u & =f, \quad & x \in \mathbb{D}, & \\
u & =g, \quad x \in \partial \mathbb{D} \backslash M .
\end{array}
$$

We introduce the functions $u_{1}(r, \varphi)=\eta(r, \varphi) \sqrt{r} \Phi_{1}(\varphi)$ and $u_{2}(r, \varphi)=\eta(r, \varphi) \sqrt{r} \Phi_{2}(\varphi)$, where $\eta$ is a cut-off function in $\mathcal{C}_{c}^{\infty}(\overline{\mathbb{D}})$ equal to 1 near the origin,

$$
\begin{aligned}
& \Phi_{1}(\varphi)=\left(\begin{array}{c}
-(5 / 2-4 \varkappa) \cos (\varphi / 2) \\
(7 / 2-4 \varkappa) \sin (\varphi / 2)
\end{array}\right)+(4 \varkappa-7 / 2)\left(\begin{array}{c}
\cos (3 \varphi / 2) \\
-\sin (3 \varphi / 2)
\end{array}\right), \\
& \Phi_{2}(\varphi)=\left(\begin{array}{c}
-(5 / 2-4 \varkappa) \sin (\varphi / 2) \\
-(7 / 2-4 \varkappa) \cos (\varphi / 2)
\end{array}\right)+(4 \varkappa-5 / 2)\left(\begin{array}{c}
\sin (3 \varphi / 2) \\
\cos (3 \varphi / 2)
\end{array}\right) .
\end{aligned}
$$

The two functions $u_{1}$ and $u_{2}$ vanish on $\partial \mathbb{D}$ and satisfy the Lamé system with righthand side of class $\mathcal{C}_{c}^{\infty}(\overline{\mathbb{D}} \backslash \mathcal{O})$. For each of these functions, the boundary integral on the left in (53) diverges because of the singularity of the integrand at the origin, while the right-hand side of (53) is finite.

In the above example, the admissibility condition (see Definition 3.1.1) fails. Moreover, the functions $u_{1}$ and $u_{2}$ do not satisfy (51).

If the opening $\alpha$ of a two-dimensional wedge $\mathbb{D}=\mathbb{K}$ is less than $2 \pi$, then this wedge is admissible. It turns out that condition (51) is also satisfied for the weak solutions of the Dirichlet problem in the case where $\alpha<2 \pi$.

To prove Proposition 3.1.2, we need the following statement. 
Lemma 3.1.4. Assume that $\nu_{j} \in \mathbb{R}, j=1, \ldots, m, \sum \nu_{j}^{2}=1$, and $b_{k} \in \mathbb{C}^{m}, k=$ $1, \ldots, m$. Then

$$
2 \sum_{j=1}^{m}\left|b_{j}\right|^{2}+2\left|\sum_{j=1}^{m} \nu_{j} b_{j}\right|^{2}=\sum_{j, k=1}^{m}\left|\nu_{k} b_{j}+\nu_{j} b_{k}\right|^{2} .
$$

Proof. We have

$$
\begin{aligned}
\sum_{j, k=1}^{m}\left|\nu_{k} b_{j}+\nu_{j} b_{k}\right|^{2}=\sum_{j, k=1}^{m}\left\langle\nu_{k} b_{j}+\nu_{j} b_{k}, \nu_{k} b_{j}+\nu_{j} b_{k}\right\rangle \\
=\sum_{j, k=1}^{m} \nu_{j} \nu_{j}\left\langle b_{k}, b_{k}\right\rangle+\sum_{j, k=1}^{m} \nu_{k} \nu_{k}\left\langle b_{j}, b_{j}\right\rangle \\
\quad+\sum_{j, k=1}^{m} \nu_{j} \nu_{k}\left\langle b_{k}, b_{j}\right\rangle+\sum_{j, k=1}^{m} \nu_{k} \nu_{j}\left\langle b_{j}, b_{k}\right\rangle \\
=2 \sum_{j=1}^{m} \nu_{j}^{2} \sum_{k=1}^{m}\left|b_{k}\right|^{2}+2 \operatorname{Re} \sum_{j, k=1}^{m} \nu_{j} \nu_{k}\left\langle b_{j}, b_{k}\right\rangle \\
=2 \sum_{k=1}^{m}\left|b_{k}\right|^{2}+2 \operatorname{Re} \sum_{j, k=1}^{m}\left\langle\nu_{j} b_{j}, \nu_{k} b_{k}\right\rangle \\
=2 \sum_{k=1}^{m}\left|b_{k}\right|^{2}+2\left\langle\sum_{j=1}^{m} \nu_{j} b_{j}, \sum_{k=1}^{m} \nu_{k} b_{k}\right\rangle \\
=2 \sum_{k=1}^{m}\left|b_{k}\right|^{2}+2\left|\sum_{j=1}^{m} \nu_{j} b_{j}\right|^{2} .
\end{aligned}
$$

Proof of Proposition 3.1.2. In (46), we choose $\left\{f^{\alpha}\right\}=\left\{-l_{1}, \ldots,-l_{n}, 0, \ldots, 0, l_{m+1}\right\}$, where $l=\left(l_{1}, \ldots, l_{n}\right)$ is a vector occurring in (50), $l_{m+1}$ is a positive number satisfying

$$
2|l|<l_{m+1} \min \left\{1, c_{0} /\left(4 c_{1}\right)\right\},
$$

and $c_{0}$ and $c_{1}$ are the constants in (36) and (20). Let $h$ be the vector in $\mathbb{R}^{m}$ with components $\left(-l_{1}, \ldots,-l_{n}, 0, \ldots, 0\right)$. We also put $u(x, t)=\chi(t) v(x)$ in (46), assuming that $v \in \mathfrak{M}$ and $\chi$ is a function as in Proposition 2.0.2. We integrate identity (46) over $\mathbb{D} \times \mathbb{R}$; since $v \in \mathfrak{M}$, we can apply the Gauss-Ostrogradskir theorem for the second term on the right-hand side:

$$
\begin{aligned}
2 \operatorname{Re} & \int_{-\infty}^{+\infty} \int_{\mathbb{D}} e^{-2 \gamma t} f^{\sigma} \overline{\partial_{\sigma} u_{i}} \partial_{\alpha}\left(g_{i j}^{\alpha \beta} \partial_{\beta} u_{j}\right) \mathrm{d} x \mathrm{~d} t \\
= & \int_{-\infty}^{+\infty} \int_{\mathbb{D}} e^{-2 \gamma t} f^{\sigma}\left(\partial_{\sigma} g_{i j}^{\alpha \beta}\right) \partial_{\alpha} u_{i} \overline{\partial_{\beta} u_{j}} \mathrm{~d} x \mathrm{~d} t \\
& +\operatorname{Re} \int_{-\infty}^{+\infty} \int_{\partial \mathbb{D}} e^{-2 \gamma t} \nu_{\alpha}\left(2 f^{\sigma} \overline{\partial_{\sigma} u_{i}} g_{i j}^{\alpha \beta} \partial_{\beta} u_{j}-f^{\alpha} g_{i j}^{\sigma \beta} \partial_{\sigma} u_{i} \overline{\partial_{\beta} u_{j}}\right) \mathrm{d} S \mathrm{~d} t \\
& +2 \gamma \operatorname{Re} \int_{-\infty}^{+\infty} \int_{\mathbb{D}} e^{-2 \gamma t}\left(2 f^{\sigma} \overline{\partial_{\sigma} u_{i}} \partial_{t} u_{i}-f^{m+1} g_{i j}^{\alpha \beta} \partial_{\alpha} u_{i} \overline{\partial_{\beta} u_{j}}\right) \mathrm{d} x \mathrm{~d} t ;
\end{aligned}
$$


here $\nu_{\alpha}$ denotes the collection $\left\{\nu_{1}, \ldots, \nu_{m}, 0\right\}$, where $\nu=\left(\nu_{1}, \ldots, \nu_{m}\right)$ is the outward unit normal to $\partial \mathbb{D}$, and $\nu_{n+1}=\cdots=\nu_{m}=0$. We rewrite (55) as follows:

$$
\begin{aligned}
& 2 \operatorname{Re} \int_{-\infty}^{+\infty} \int_{\mathbb{D}} e^{-2 \gamma t} f^{\sigma} \overline{\partial_{\sigma} u_{i}} \partial_{\alpha}\left(g_{i j}^{\alpha \beta} \partial_{\beta} u_{j}\right) \mathrm{d} x \mathrm{~d} t \\
& =\int_{-\infty}^{+\infty} \int_{\mathbb{D}} e^{-2 \gamma t} f^{\sigma}\left(\partial_{\sigma} g_{i j}^{\alpha \beta}\right) \partial_{\alpha} u_{i} \overline{\partial_{\beta} u_{j}} \mathrm{~d} x \mathrm{~d} t \\
& \quad-\operatorname{Re} \int_{-\infty}^{+\infty} \int_{\partial \mathbb{D}} e^{-2 \gamma t}\left(\langle\nu, h\rangle\left|\partial_{t} u\right|^{2}+2 f^{\sigma} \overline{\partial_{\sigma} u_{i}} a_{i j}^{k l} \nu_{k} \partial_{l} u_{j}\right. \\
& \left.\quad-\langle\nu, h\rangle a_{i j}^{k l} \partial_{k} u_{i} \overline{\partial_{l} u_{j}}\right) \mathrm{d} S \mathrm{~d} t \\
& +2 \gamma \operatorname{Re} \int_{-\infty}^{+\infty} \int_{\mathbb{D}} e^{-2 \gamma t}\left(f^{m+1}\left|\partial_{t} u\right|^{2}+2 h_{k} \overline{\partial_{k} u_{i}} \partial_{t} u_{i}\right. \\
& \left.+f^{m+1} a_{i j}^{k l} \partial_{k} u_{i} \overline{\partial_{l} u_{j}}\right) \mathrm{d} x \mathrm{~d} t .
\end{aligned}
$$

To transform the surface integral, we put

$$
h=-\zeta(x) \nu+\xi, \quad \nabla u_{i}=t_{i} \nu+\sigma_{i}, \quad i=1, \ldots, m,
$$

where $\xi$ and the $\sigma_{i}$ are the components tangent to $\partial \mathbb{D}, t_{i}=\partial_{\nu} u_{i}$. In terms of projections onto the coordinate axes, these expressions take the form

$$
h_{k}=-\zeta(x) \nu_{k}+\xi_{k}, \quad \partial_{k} u_{i}=t_{i} \nu_{k}+\sigma_{k i}, \quad i, k=1, \ldots, m .
$$

Since the wedge $\mathbb{D}$ is admissible, we have $\zeta(x) \geq c>0$ for $x \in \partial \mathbb{D} \backslash M$. We can write

$$
\begin{aligned}
-2 & f^{\sigma} \overline{\partial_{\sigma} u_{i}} a_{i j}^{k l} \partial_{l} u_{j} \nu_{k}+\zeta\left|\partial_{t} u\right|^{2}-\zeta a_{i j}^{k l} \partial_{k} u_{i} \overline{\partial_{l} u_{j}} \\
= & -2 f^{m+1} \overline{\partial_{t} u_{i}} a_{i j}^{k l} \nu_{k}\left(t_{j} \nu_{l}+\sigma_{l j}\right)-2 h_{p}\left(\overline{t_{i}} \nu_{p}+\overline{\sigma_{p i}}\right) a_{i j}^{k l} \nu_{k}\left(t_{j} \nu_{l}+\sigma_{l j}\right) \\
& +\zeta\left|\partial_{t} u\right|^{2}-\zeta a_{i j}^{k l}\left(t_{i} \nu_{k}+\sigma_{k i}\right)\left(\overline{t_{j}} \nu_{l}+\overline{\sigma_{l j}}\right) \\
= & -2 f^{m+1} \overline{\partial_{t} u_{i}} a_{i j}^{k l} \nu_{k} t_{j} \nu_{l}-2 f^{m+1} \overline{\partial_{t} u_{i}} a_{i j}^{k l} \nu_{k} \sigma_{l j}+\zeta a_{i j}^{k l} \nu_{k} \overline{t_{i}} \nu_{l} t_{j} \\
& +2 \zeta a_{i j}^{k l} \overline{t_{i}} \nu_{k} \sigma_{l j}-2 h_{p} \overline{\sigma_{p i}} \nu_{k} a_{i j}^{k l} t_{j} \nu_{l}-2 h_{p} \overline{\sigma_{p i}} \nu_{k} a_{i j}^{k l} \sigma_{l j} \\
& +\zeta\left|\partial_{t} u\right|^{2}-2 \zeta \operatorname{Re}\left(a_{i j}^{k l} \sigma_{k i} \overline{\bar{t}_{j}} \nu_{l}\right)-\zeta a_{i j}^{k l} \sigma_{k i} \overline{\sigma_{l j}} .
\end{aligned}
$$

Taking the properties of the tensor $a_{i j}^{k l}$ into account, we symmetrize all the terms involving the factor $t_{i} \nu_{l}$ :

$$
\begin{aligned}
& -f^{m+1} \overline{\partial_{t} u_{i}} a_{i j}^{k l} \nu_{k}\left(t_{j} \nu_{l}+t_{l} \nu_{j}\right)-2 f^{m+1} \overline{\partial_{t} u_{i}} a_{i j}^{k l} \nu_{k} \sigma_{l j} \\
& +(1 / 4) \zeta a_{i j}^{k l} \overline{\left(\nu_{k} t_{i}+\nu_{i} t_{k}\right)}\left(\nu_{l} t_{j}+\nu_{j} t_{l}\right)+\zeta a_{i j}^{k l} \overline{\left(t_{i} \nu_{k}+t_{k} \nu_{i}\right)} \sigma_{l j} \\
& -h_{p} \overline{\sigma_{p i}} \nu_{k} a_{i j}^{k l}\left(t_{j} \nu_{l}+t_{l} \nu_{j}\right)-2 h_{p} \overline{\sigma_{p i}} \nu_{k} a_{i j}^{k l} \sigma_{l j}+\zeta\left|\partial_{t} u\right|^{2} \\
& -\zeta \operatorname{Re}\left(a_{i j}^{k l} \sigma_{k i} \overline{\left(t_{j} \nu_{l}+t_{l} \nu_{j}\right)}\right)-\zeta a_{i j}^{k l} \sigma_{k i} \overline{\sigma_{l j}} .
\end{aligned}
$$

Using the inequalities $\zeta(x) \geq c>0$ and (36), we obtain

$$
\zeta\left|\partial_{t} u\right|^{2}+(\zeta / 4) a_{i j}^{k l} \overline{\left(t_{k} \nu_{i}+t_{i} \nu_{k}\right)}\left(t_{l} \nu_{j}+t_{j} \nu_{l}\right) \geq c\left(\left|\partial_{t} u\right|^{2}+\sum_{i, j}\left|t_{i} \nu_{j}+t_{j} \nu_{i}\right|^{2}\right) .
$$

The remaining terms in (57) are dominated by

$$
\varepsilon \sum_{i, j}\left|t_{i} \nu_{j}+t_{j} \nu_{i}\right|^{2}+C_{\varepsilon}\left(\left|\partial_{t} u\right|^{2}+\sum_{k, l}\left|\sigma_{k l}\right|^{2}\right)
$$


with sufficiently small $\varepsilon$. Thus,

$$
\begin{aligned}
& \gamma \int_{-\infty}^{\infty} \int_{\mathbb{D}} e^{-2 \gamma t}\left(f^{m+1}\left|\partial_{t} u\right|^{2}+f^{m+1} a_{i j}^{k l} \partial_{k} u_{i} \overline{\partial_{l} u_{j}}+2 \operatorname{Re}\left(h_{k} \partial_{t} u_{i} \overline{\partial_{k} u_{i}}\right)\right) \mathrm{d} x \mathrm{~d} t \\
&+\int_{-\infty}^{+\infty} \int_{\partial \mathbb{D}} e^{-2 \gamma t} \sum_{i, j}\left|t_{i} \nu_{j}+t_{j} \nu_{i}\right|^{2} \mathrm{~d} S \mathrm{~d} t \\
& \leq c\left\{2 \operatorname{Re} \int_{-\infty}^{+\infty} \int_{\mathbb{D}} e^{-2 \gamma t} f^{\sigma} \overline{\partial_{\sigma} u_{i}} \partial_{\alpha}\left(g_{i j}^{\alpha \beta} \partial_{\beta} u_{j}\right) \mathrm{d} x \mathrm{~d} t\right. \\
&+ \int_{-\infty}^{+\infty} \int_{\mathbb{D}} e^{-2 \gamma t}\left(\left|\partial_{t} u\right|^{2}+|\nabla u|^{2}\right) \mathrm{d} x \mathrm{~d} t \\
&+\left.\int_{-\infty}^{+\infty} \int_{\partial \mathbb{D}} e^{-2 \gamma t}\left(\varepsilon \sum_{i, j}\left|t_{i} \nu_{j}+t_{j} \nu_{i}\right|^{2}+C_{\varepsilon}\left(\left|\partial_{t} u\right|^{2}+\sum_{k, l}\left|\sigma_{k l}\right|^{2}\right)\right) \mathrm{d} S \mathrm{~d} t\right\} .
\end{aligned}
$$

Consider the first integral in (158). We symmetrize $a_{i j}^{k l} \partial_{k} u_{i} \overline{\partial_{l} u_{j}}$ and use (36) and (20) to obtain

$$
\begin{gathered}
\int_{\mathbb{D}} a_{i j}^{k l} \partial_{k} u_{i} \overline{\partial_{l} u_{j}} \mathrm{~d} x=\frac{1}{4} \int_{\mathbb{D}} a_{i j}^{k l}\left(\partial_{k} u_{i}+\partial_{i} u_{k}\right) \overline{\left(\partial_{l} u_{j}+\partial_{j} u_{l}\right)} \mathrm{d} x \\
\geq\left(c_{0} / 4\right) \int_{\mathbb{D}} \sum_{i, j}\left|\partial_{i} u_{j}+\partial_{j} u_{i}\right|^{2} \mathrm{~d} x \geq\left(c_{0} / 4 c_{1}\right) \int_{\mathbb{D}}\left|\nabla_{x} u\right|^{2} \mathrm{~d} x
\end{gathered}
$$

where $c_{0}$ and $c_{1}$ are the constants in (36) and (20). Furthermore,

$$
2\left|\operatorname{Re} \int_{\mathbb{D}} h_{k} \overline{\partial_{k} u_{i}} \partial_{t} u_{i} \mathrm{~d} x\right| \leq|l| \int_{\mathbb{D}}\left(\left|\nabla_{x} u\right|^{2}+\left|\partial_{t} u\right|^{2}\right) \mathrm{d} x .
$$

Therefore, by (54),

$$
\begin{gathered}
\gamma \int_{-\infty}^{\infty} \int_{\mathbb{D}} e^{-2 \gamma t}\left(f^{m+1}\left|\partial_{t} u\right|^{2}+f^{m+1} a_{i j}^{k l} \partial_{k} u_{i} \overline{\partial_{l} u_{j}}+2 \operatorname{Re}\left(h_{k} \partial_{t} u_{i} \overline{\partial_{k} u_{i}}\right)\right) \mathrm{d} x \mathrm{~d} t \\
\geq \gamma \int_{-\infty}^{\infty} \int_{\mathbb{D}} e^{-2 \gamma t}\left(f^{m+1}\left|\partial_{t} u\right|^{2}+f^{m+1}\left(c_{0} / 4 c_{1}\right)\left(\left|\nabla_{x} u\right|^{2}+\left|u_{t}\right|^{2}\right)\right. \\
\left.\quad-|l|\left(\left|\nabla_{x} u\right|^{2}+\left|u_{t}\right|^{2}\right)\right) \mathrm{d} x \mathrm{~d} t \\
\geq c \gamma \int_{-\infty}^{\infty} \int_{\mathbb{D}} e^{-2 \gamma t}\left(\left|\nabla_{x} u\right|^{2}+\left|u_{t}\right|^{2}\right) \mathrm{d} x \mathrm{~d} t .
\end{gathered}
$$

For the first term on the right in (58) we have

$$
\begin{aligned}
2 & \operatorname{Re} \int_{-\infty}^{+\infty} \int_{\mathbb{D}} e^{-2 \gamma t} f^{\sigma} \overline{\partial_{\sigma} u_{i}} \partial_{\alpha}\left(g_{i j}^{\alpha \beta} \partial_{\beta} u_{j}\right) \mathrm{d} x \mathrm{~d} t \\
& \leq\left\{\frac{1}{\varepsilon \gamma} \int_{-\infty}^{+\infty} \int_{\mathbb{D}} e^{-2 \gamma t}\left|\mathcal{L}\left(x, D_{x}, D_{t}\right) u\right|^{2} \mathrm{~d} x \mathrm{~d} t+\gamma \varepsilon \int_{-\infty}^{+\infty} \int_{\mathbb{D}} e^{-2 \gamma t}\left|\nabla_{x, t} u\right|^{2} \mathrm{~d} x \mathrm{~d} t\right\}
\end{aligned}
$$


with sufficiently small $\varepsilon$. Taken together, these estimates and Lemma 3.1.4 lead to the inequality

$$
\begin{aligned}
& \gamma^{2} \int_{-\infty}^{+\infty} \int_{\mathbb{D}} e^{-2 \gamma t}\left|\nabla_{x, t} u\right|^{2} \mathrm{~d} x \mathrm{~d} t+\gamma \int_{-\infty}^{+\infty} \int_{\partial \mathbb{D}} e^{-2 \gamma t}\left|\partial_{\nu} u\right|^{2} \mathrm{~d} S \mathrm{~d} t \\
& \leq c\left\{\int_{-\infty}^{+\infty} \int_{\mathbb{D}} e^{-2 \gamma t}\left|\mathcal{L}\left(x, D_{x}, D_{t}\right) u\right| \mathrm{d} x \mathrm{~d} t\right. \\
&\left.\quad+\gamma \int_{-\infty}^{+\infty} \int_{\partial \mathbb{D}} e^{-2 \gamma t}\left(\left|\partial_{t} u\right|^{2}+\left|\nabla_{\sigma} u\right|^{2}\right) \mathrm{d} S \mathrm{~d} t+\gamma \int_{-\infty}^{+\infty} \int_{\mathbb{D}} e^{-2 \gamma t}\left|\nabla_{x, t} u\right|^{2} \mathrm{~d} x \mathrm{~d} t\right\},
\end{aligned}
$$

where $\nabla_{\sigma}$ denotes the tangent component of the gradient. For large $\gamma$, we can remove the last term from the right-hand side at the expense of increasing the constant $c$. To get (53), it remains to take into account the Parseval identity and the arbitrariness of $\chi$.

Remark 3.1.5. If the tensor $a_{i j}^{k l}$ is independent of $x$, then inequality (53) is valid for any $\gamma>0$. Indeed, if $a_{i j}^{k l}=$ const, then the first term on the right in (55) is absent, and the integral

$$
\gamma \int_{-\infty}^{+\infty} \int_{\mathbb{D}} e^{-2 \gamma t}\left|\nabla_{x, t} u\right|^{2} \mathrm{~d} x \mathrm{~d} t
$$

on the right-hand side of (59) disappears. Therefore, inequality (53) is valid not only for large but for all positive $\gamma$.

3.2. Estimates of solutions with Neumann boundary condition. When estimating solutions with Dirichlet condition, we required that the wedge $\mathbb{D}=\mathbb{K} \times \mathbb{R}^{m-n}$ be admissible (see Definition 3.1.1). In the case of a Neumann condition, we can do without that requirement and consider an arbitrary cone $\mathbb{K}$ smooth outside the vertex.

We write a point $x \in \mathbb{D}$ in the form $x=(y, z) \in \mathbb{K} \times \mathbb{R}^{m-n}$, where $y=\left(y_{1}, \ldots, y_{n}\right)$ and $z=\left(z_{1}, \ldots, z_{m-n}\right)$. We introduce $H_{0}^{1}(\mathbb{D})$ as the completion of $\mathcal{C}_{c}^{\infty}(\overline{\mathbb{D}} \backslash M)$ in the norm

$$
\left\|u ; H_{0}^{1}(\mathbb{D})\right\|=\left(\int_{\mathbb{D}}\left(\left|\nabla_{x} u(x)\right|^{2}+|y|^{-2}|u(x)|^{2}\right) \mathrm{d} x\right)^{1 / 2}
$$

Let $H_{0}^{1 / 2}(\partial \mathbb{D})$ be the space of traces on $\partial \mathbb{D}$ of the functions in $H_{0}^{1}(\mathbb{D})$, and let $H_{0}^{-1 / 2}(\partial \mathbb{D})$ be the space dual to $H_{0}^{1 / 2}(\partial \mathbb{D})$ with respect to the (extended) inner product on $L_{2}(\partial \mathbb{D})$. First, we consider the case where $\operatorname{dim} \mathbb{K}=n>2$ and introduce the set

$$
\left\{v \in \mathcal{C}^{\infty}(\overline{\mathbb{D}} \backslash M) \cap H^{1}(\mathbb{D}): \mathcal{B}\left(x, D_{x}\right) v \in H_{0}^{-1 / 2}(\partial \mathbb{D}), \mathcal{L}\left(x, D_{x}, 0\right) v \in L_{2}(\mathbb{D})\right\}
$$

where $\left(\mathcal{B}\left(x, D_{x}\right) u\right)_{i}=-\nu_{\alpha} g_{i j}^{\alpha \beta} D_{\beta} u_{j}$.

Proposition 3.2.1. Let $\mathbb{D}=\mathbb{K} \times \mathbb{R}^{m-n}$ be a wedge in $\mathbb{R}^{m}$ with $\operatorname{dim} \mathbb{K}=n>2$. For the functions in the set (60), we have

$$
\begin{aligned}
& \gamma^{2} \int_{\mathbb{D}}\left(\left|\nabla_{x} v(x)\right|^{2}+|\tau|^{2} \cdot|v(x)|^{2}\right) \mathrm{d} x \\
& \quad \leq c\left\{\int_{\mathbb{D}}\left|\mathcal{L}\left(x, D_{x}, \tau\right) v(x)\right|^{2} \mathrm{~d} x+|\tau|^{2}\left\|\mathcal{B}\left(x, D_{x}\right) v ; H_{0}^{-1 / 2}(\partial \mathbb{D})\right\|^{2}\right\}
\end{aligned}
$$

with constant $c$ independent of $v$ and $\tau=\sigma-i \gamma$, where $\sigma \in \mathbb{R}, \gamma>0$. 
Proof. For the role of $\left\{f^{\alpha}\right\}_{\alpha=1}^{m+1}$ in (46), we choose the collection $\left\{f^{\alpha}\right\}=\left\{0, \ldots, 0, f^{m+1}\right\}$ with $f^{m+1}=$ const $\neq 0$. Then (55) takes the form

$$
\begin{aligned}
\operatorname{Re} & \int_{-\infty}^{+\infty} \int_{\mathbb{D}} e^{-2 \gamma t} \overline{\partial_{t} u_{i}}\left(\mathcal{L}\left(x, D_{x}, D_{t}\right) u\right)_{i} \mathrm{~d} x \mathrm{~d} t \\
= & -\operatorname{Re} i \int_{-\infty}^{+\infty} \int_{\partial \mathbb{D}} e^{-2 \gamma t} \overline{\partial_{t} u_{i}}\left(\mathcal{B}\left(x, D_{x}\right) u\right)_{i} \mathrm{~d} S \mathrm{~d} t \\
& +\gamma \int_{-\infty}^{+\infty} \int_{\mathbb{D}} e^{-2 \gamma t}\left(a_{i j}^{k l} \partial_{k} u_{i} \overline{\partial_{l} u_{j}}+\left|\partial_{t} u\right|^{2}\right) \mathrm{d} x \mathrm{~d} t
\end{aligned}
$$

Here $u(x, t)=v(x) \chi(t)$, the functions $t \mapsto \chi(t)$ and $t \mapsto e^{-\gamma t} \chi(t)$ belong to the Schwartz class $\mathcal{S}(\mathbb{R})$, and $v$ is in the set (60). Hence,

$$
\begin{aligned}
\gamma^{2} \int_{-\infty}^{+\infty} & \int_{\mathbb{D}} e^{-2 \gamma t}\left(a_{i j}^{k l} \partial_{k} u_{i} \overline{\partial_{l} u_{j}}+\left|\partial_{t} u\right|^{2}\right) \mathrm{d} x \mathrm{~d} t \\
\leq c & \left\{\int_{-\infty}^{+\infty} \int_{\mathbb{D}} e^{-2 \gamma t}\left|\mathcal{L}\left(x, D_{x}, D_{t}\right) u\right|^{2} \mathrm{~d} x \mathrm{~d} t\right. \\
& \left.\quad+\gamma \operatorname{Re} i \int_{-\infty}^{+\infty} \int_{\partial \mathbb{D}} e^{-2 \gamma t} \overline{\partial_{t} u_{j}}\left(\mathcal{B}\left(x, D_{x}\right) u\right)_{j} \mathrm{~d} S \mathrm{~d} t\right\} .
\end{aligned}
$$

By (16), (36), and (20), we have

$$
\int_{-\infty}^{+\infty} \int_{\mathbb{D}} e^{-2 \gamma t}\left|\nabla_{x} u(x, t)\right|^{2} \mathrm{~d} x \mathrm{~d} t \leq c \int_{-\infty}^{+\infty} \int_{\mathbb{D}} e^{-2 \gamma t} a_{i j}^{k l} \partial_{k} u_{i} \overline{\partial_{l} u_{j}} \mathrm{~d} x \mathrm{~d} t
$$

Integrating by parts, we obtain

$$
\begin{aligned}
\int_{-\infty}^{+\infty} \int_{\partial \mathbb{D}} e^{-2 \gamma t} \overline{\partial_{t} u_{i}}\left(\mathcal{B}\left(x, D_{x}\right) u\right)_{i} \mathrm{~d} S \mathrm{~d} t \\
=2 \gamma \int_{-\infty}^{+\infty} \int_{\partial \mathbb{D}} e^{-2 \gamma t} \overline{u_{i}}\left(\mathcal{B}\left(x, D_{x}\right) u\right)_{i} \mathrm{~d} S \mathrm{~d} t \\
\quad-\int_{-\infty}^{+\infty} \int_{\partial \mathbb{D}} e^{-2 \gamma t} \overline{u_{i}} \partial_{t}\left(\mathcal{B}\left(x, D_{x}\right) u\right)_{i} \mathrm{~d} S \mathrm{~d} t
\end{aligned}
$$

Since

$$
\left|\int_{\partial \mathbb{D}} \varphi \cdot \bar{\psi} \mathrm{d} S\right| \leq\left\|\varphi ; H_{0}^{1 / 2}(\partial \mathbb{D})\right\| \cdot\left\|\psi ; H_{0}^{-1 / 2}(\partial \mathbb{D})\right\|
$$

from (63) we deduce that

$$
\begin{aligned}
\gamma^{2} \int_{-\infty}^{+\infty} \int_{\mathbb{D}} e^{-2 \gamma t}\left|\nabla_{x, t} u\right|^{2} \mathrm{~d} x \mathrm{~d} t \\
\leq c\left\{\varepsilon \gamma^{2} \int_{-\infty}^{+\infty} e^{-2 \gamma t}\left\|u ; H_{0}^{\frac{1}{2}}(\partial \mathbb{D})\right\|^{2} \mathrm{~d} t\right. \\
\quad+\int_{-\infty}^{+\infty} \int_{\mathbb{D}} e^{-2 \gamma t}\left|\mathcal{L}\left(x, D_{x}, D_{t}\right) u\right|^{2} \mathrm{~d} x \mathrm{~d} t \\
\left.\quad+C_{\varepsilon} \int_{-\infty}^{+\infty} e^{-2 \gamma t}\left(\gamma^{2}\left\|\mathcal{B}\left(x, D_{x}\right) u ; H_{0}^{-\frac{1}{2}}(\partial \mathbb{D})\right\|^{2}+\left\|\partial_{t} \mathcal{B}\left(x, D_{x}\right) u ; H_{0}^{-\frac{1}{2}}(\partial \mathbb{D})\right\|^{2}\right) \mathrm{d} t\right\} .
\end{aligned}
$$

Put $v(x)=v(r, \phi, z)$ with $r=|y|$ and $\phi=y /|y|$. If $\operatorname{dim} \mathbb{K}=n>2$, then the Hardy inequality

$$
\int_{0}^{+\infty}|v(r, \phi, z)|^{2} r^{n-3} \mathrm{~d} r \leq \frac{4}{(n-2)^{2}} \int_{0}^{+\infty}\left|\partial_{r} v(r, \phi, z)\right|^{2} r^{n-1} \mathrm{~d} r
$$


shows that

$$
\int_{\mathbb{K}}|v(y, z)|^{2}|y|^{-2} \mathrm{~d} y \leq c \int_{\mathbb{K}}\left|\nabla_{y} v(y, z)\right|^{2} \mathrm{~d} y .
$$

Therefore,

$$
\left\|u ; H_{0}^{1 / 2}(\partial \mathbb{D})\right\|^{2} \leq c_{1}\left\|u ; H_{0}^{1}(\mathbb{D})\right\|^{2} \leq c_{2}\left\|\nabla_{x} u ; L_{2}(\mathbb{D})\right\|^{2} .
$$

Now, formula (65) with sufficiently small $\varepsilon$ implies that

$$
\begin{aligned}
\gamma^{2} \int_{-\infty}^{+\infty} \int_{\mathbb{D}} e^{-2 \gamma t}\left|\nabla_{x, t} u\right|^{2} \mathrm{~d} x \mathrm{~d} t & \\
\leq c & \left\{\int_{-\infty}^{+\infty} \int_{\mathbb{D}} e^{-2 \gamma t}\left|\mathcal{L}\left(x, D_{x}, D_{t}\right) u\right|^{2} \mathrm{~d} x \mathrm{~d} t\right. \\
& +\int_{-\infty}^{+\infty} e^{-2 \gamma t}\left(\gamma^{2}\left\|\mathcal{B}\left(x, D_{x}\right) u ; H_{0}^{-1 / 2}(\partial \mathbb{D})\right\|^{2}\right. \\
& \left.\left.+\left\|\partial_{t} \mathcal{B}\left(x, D_{x}\right) u ; H_{0}^{-1 / 2}(\partial \mathbb{D})\right\|^{2}\right) \mathrm{~d} t\right\} .
\end{aligned}
$$

It remains to use the Parseval identity for the Fourier transformation $\mathcal{F}_{t \rightarrow \tau}$ and to recall that the function $\chi$ is arbitrary.

Now, let $\operatorname{dim} \mathbb{K}=2$; we introduce the set

$$
\left\{v \in \mathcal{C}^{\infty}(\overline{\mathbb{D}} \backslash M) \cap H^{1}(\mathbb{D}): \mathcal{B}\left(x, D_{x}\right) v \in H^{-1 / 2}(\partial \mathbb{D}), \mathcal{L}\left(x, D_{x}, 0\right) v \in L_{2}(\mathbb{D})\right\} .
$$

In contrast to (60), this definition involves the spaces $H^{1}(\mathbb{D})$ and $H^{-1 / 2}(\partial \mathbb{D})$ instead of the weighted spaces $H_{0}^{1}(\mathbb{D})$ and $H_{0}^{-1 / 2}(\partial \mathbb{D})$. The reason is that the Hardy inequality fails for $n=2$, so that the estimate for solutions needs modification.

Proposition 3.2.2. Let $\mathbb{D}=\mathbb{K} \times \mathbb{R}^{m-n}$ be a wedge in $\mathbb{R}^{m}$ with $\operatorname{dim} \mathbb{K}=2$. For the functions in the set (66), we have

$$
\begin{aligned}
& \gamma^{2}\left(\left\|\nabla_{x} v ; L_{2}(\mathbb{D})\right\|^{2}+|\tau|^{2}\left\|v ; L_{2}(\mathbb{D})\right\|^{2}\right) \mathrm{d} x \\
& \quad \leq c\left\{\left\|\mathcal{L}\left(x, D_{x}, \tau\right) v ; L_{2}(\mathbb{D})\right\|^{2}+|\tau|^{2}\left\|\mathcal{B}\left(x, D_{x}\right) v ; H^{-1 / 2}(\partial \mathbb{D})\right\|^{2}\right\}
\end{aligned}
$$

where $\tau=\sigma-i \gamma$ with $\sigma \in \mathbb{R}$ and $\gamma \geq \gamma_{0}$. Here $\gamma_{0}$ is any fixed positive number, and the constant $c$ depends on $\gamma_{0}$ and is independent of $v$ and $\tau$.

Proof. To verify this, we can slightly modify the proof of Proposition 3.2.1. Namely, instead of (64), we apply a similar estimate for the usual Sobolev spaces. Then from (63) it follows that

$$
\begin{aligned}
\gamma^{2} \int_{-\infty}^{+\infty} \int_{\mathbb{D}} e^{-2 \gamma t}\left|\nabla_{x, t} u\right|^{2} \mathrm{~d} x \mathrm{~d} t \\
\leq c\left\{\varepsilon \gamma^{2} \int_{-\infty}^{+\infty} e^{-2 \gamma t}\left\|u ; H^{1 / 2}(\partial \mathbb{D})\right\|^{2} \mathrm{~d} t\right. \\
\quad+\int_{-\infty}^{+\infty} \int_{\mathbb{D}} e^{-2 \gamma t}\left|\mathcal{L}\left(x, D_{x}, D_{t}\right) u\right|^{2} \mathrm{~d} x \mathrm{~d} t \\
\left.\quad+C_{\varepsilon} \int_{-\infty}^{+\infty} e^{-2 \gamma t}\left(\gamma^{2}\left\|\mathcal{B}\left(x, D_{x}\right) u ; H^{-1 / 2}(\partial \mathbb{D})\right\|^{2} \mathrm{~d} t+\left\|\partial_{t} \mathcal{B}\left(x, D_{x}\right) u ; H^{-1 / 2}(\partial \mathbb{D})\right\|^{2}\right) \mathrm{d} t\right\} .
\end{aligned}
$$


Furthermore,

$$
\begin{aligned}
& \int_{-\infty}^{\infty} e^{-2 \gamma t}\left\|u ; H^{1 / 2}(\partial \mathbb{D})\right\|^{2} \mathrm{~d} t \leq c \int_{-\infty}^{\infty} e^{-2 \gamma t}\left\|u ; H^{1}(\mathbb{D})\right\|^{2} \mathrm{~d} t \\
& \quad \leq c \int_{-\infty}^{\infty} e^{-2 \gamma t}\left(\left\|\nabla_{x} u ; L_{2}(\mathbb{D})\right\|^{2}+\left(1 / \gamma^{2}\right)\left\|\partial_{t} u ; L_{2}(\mathbb{D})\right\|^{2}\right) \mathrm{d} t \\
& \quad \leq c \int_{-\infty}^{\infty} \int_{\mathbb{D}} e^{-2 \gamma t}\left|\nabla_{x, t} u(x, t)\right|^{2} \mathrm{~d} t \mathrm{~d} x
\end{aligned}
$$

At the last step we used the condition $\gamma \geq \gamma_{0}$. We replace the first term on the right in (68) by the majorant obtained. For small $\varepsilon$, this new term can be eliminated at the expense of increasing the constant $c$. After that, we complete the proof as in Proposition 3.2 .1

\section{§4. Strong SOlutions}

4.1. The Dirichlet problem with homogeneous energy estimate in a wedge. With the boundary value problem

$$
\begin{aligned}
\mathcal{L}\left(x, D_{x}, \tau\right) v(x) & =f(x), & & x \in \mathbb{D}, \\
v(x) & =0, & & x \in \partial \mathbb{D} \backslash M,
\end{aligned}
$$

we associate the unbounded operator $v \mapsto \mathcal{A}(\tau) v:=\mathcal{L}\left(x, D_{x}, \tau\right) v$ on $L_{2}(\mathbb{D})$, with the domain

$$
\left\{v \in C^{\infty}(\overline{\mathbb{D}} \backslash M) \cap H^{1}(\mathbb{D}):\left.v\right|_{\partial \mathbb{D} \backslash M}=0, \mathcal{L}\left(x, D_{x}, \tau\right) v \in L_{2}(\mathbb{D})\right\} ;
$$

the condition $\mathcal{L}\left(x, D_{x}, \tau\right) v \in L_{2}(\mathbb{D})$ can be replaced by $\mathcal{L}\left(x, D_{x}, 0\right) v \in L_{2}(\mathbb{D})$.

The operator $\mathcal{A}(\tau)$ admits closure. Indeed, let $v_{n} \in \mathcal{D}(\mathcal{A}(\tau))$ be such that $v_{n} \rightarrow v$, and $\mathcal{A}(\tau) v_{n} \rightarrow f$ (both sequences converge in $\left.L_{2}(\mathbb{D})\right)$. It is obvious that $\left(\mathcal{A}(\tau) v_{n}, w\right)=$ $\left(v_{n}, \mathcal{A}(\bar{\tau}) w\right)$ for all $w \in C_{c}^{\infty}(\mathbb{D})$. If $v_{n} \rightarrow 0$, then $(f, w)=0$ and $f=0$, as required. In what follows, $\mathcal{A}(\tau)$ and $\mathcal{D}(\mathcal{A}(\tau))$ denote the closed operator and its domain.

Definition 4.1.1. A solution of the equation $\mathcal{A}(\tau) v=f$ is called a strong solution of the boundary value problem (69), (70).

Theorem 4.1.2. For every $f \in L_{2}(\mathbb{D})$ and every $\tau=\sigma-i \gamma$ with $\sigma \in \mathbb{R}$ and $\gamma>0$, there exists a unique strong solution $v(=v(\cdot, \tau))$ of problem (69), (70). The estimate

$$
\gamma^{2} \int_{\mathbb{D}}\left(|\tau|^{2}|v(x)|^{2}+\left|\nabla_{x} v(x)\right|^{2}\right) \mathrm{d} x \leq c \int_{\mathbb{D}}|f(x)|^{2} \mathrm{~d} x
$$

is true with constant $c$ independent of $\tau$.

Before verifying the theorem, we establish the existence of a weak solution of problem (69), (70). We introduce the quadratic form

$$
B(u, v):=\int_{\mathbb{D}} \sum_{i, j, k, l} a_{i j}^{k l}(x) \partial_{k} u_{i}(x) \overline{\partial_{l} v_{j}(x)} \mathrm{d} x-\tau^{2} \int_{\mathbb{D}} u(x) \overline{v(x)} \mathrm{d} x
$$

on $H^{1}(\mathbb{D})$. Let $\stackrel{\circ}{H}^{1}(\mathbb{D})$ denote the completion of $C_{c}^{\infty}(\mathbb{D})$ in the norm of $H^{1}(\mathbb{D})$. An element $u \in \stackrel{\circ}{H}^{1}(\mathbb{D})$ is called a weak solution of problem (69), (70) if $B(u, v)=(f, v)$ for all $v \in \stackrel{\circ}{H}^{1}(\mathbb{D})$. The existence and uniqueness of a weak solution can be obtained from the Lax-Milgram-Vishik lemma (in the form given in [32, 2.9.3]) combined with the following assertion. 
Proposition 4.1.3 (cf. [3]). If $\tau^{2} \in \mathbb{C} \backslash \overline{\mathbb{R}}_{+}$, then

$$
|B(u, u)| \geq \delta\left\|u ; H^{1}(\mathbb{D})\right\|^{2}
$$

for all $u \in H^{1}(\mathbb{D})$, with $\delta=\delta(\tau)>0$.

Proof. We put

$$
\alpha=\int_{\mathbb{D}}|u(x)|^{2} \mathrm{~d} x, \quad \beta=\int_{\mathbb{D}} \sum_{i, j, k, l} a_{i j}^{k l}(x) \partial_{k} u_{i}(x) \overline{\partial_{l} u_{j}(x)} \mathrm{d} x .
$$

By (20), we have

$$
\begin{aligned}
& \left(\alpha^{2}+\beta^{2}\right)^{1 / 2} \geq 2^{-1 / 2}(\alpha+\beta) \\
& \quad \geq c\left(\int_{\mathbb{D}} \sum_{i, j}\left|\partial_{i} u_{j}(x)+\partial_{j} u_{i}(x)\right|^{2} \mathrm{~d} x+\left\|u ; L_{2}(\mathbb{D})\right\|^{2}\right) \geq c_{1}\left\|u ; H^{1}(\mathbb{D})\right\|^{2} .
\end{aligned}
$$

Therefore, it suffices to prove that

$$
|B(u, u)|^{2} \geq \delta\left(\alpha^{2}+\beta^{2}\right) .
$$

Putting $\tau^{2}=\sigma_{1}+i \gamma_{1}$ with real $\sigma_{1}, \gamma_{1}$, we obtain $|B(u, u)|^{2}=\beta^{2}+\left(\sigma_{1}^{2}+\gamma_{1}^{2}\right) \alpha^{2}-2 \sigma_{1} \alpha \beta$. If $\sigma_{1}=0$ and $\gamma_{1} \neq 0$ or $\sigma_{1}<0$, then (73) is clear. If $\sigma_{1}>0$, then $\gamma_{1} \neq 0$. Choose $\varepsilon \in \mathbb{R}$ such that $\varepsilon^{2}<1$ and $\left(\varepsilon^{-2}-1\right) \sigma_{1}^{2}<\gamma_{1}^{2}$. Then $2 \sigma_{1} \alpha \beta \leq \varepsilon^{2} \beta^{2}+\varepsilon^{-2} \sigma_{1}^{2} \alpha^{2}$ and

$$
|B(u, u)|^{2} \geq \beta^{2}\left(1-\varepsilon^{2}\right)+\alpha^{2}\left(\gamma_{1}^{2}-\left(\varepsilon^{-2}-1\right) \sigma_{1}^{2}\right) \geq \delta\left(\alpha^{2}+\beta^{2}\right) .
$$

Proof of the theorem. By Proposition 2.0.2, estimate (71) remains valid for the elements $v$ in $\mathcal{D}(\mathcal{A}(\tau))$. Hence, the kernel $\operatorname{Ker} \mathcal{A}(\tau)$ of the operator $\mathcal{A}(\tau)$ is trivial and the range $\mathcal{R} \mathcal{A}(\tau)$ is closed. Moreover, $C_{c}^{\infty}(\mathbb{D}) \subset \mathcal{R} \mathcal{A}(\tau)$ because the weak solution of problem (69), (70) with $f \in C_{c}^{\infty}(\mathbb{D})$ belongs to $\mathcal{D}(\mathcal{A}(\tau))$, in accordance with the theory of elliptic boundary value problems. Since $\mathcal{R} \mathcal{A}(\tau)$ is closed, we conclude that $\mathcal{R} \mathcal{A}(\tau)=L_{2}(\mathbb{D})$.

4.2. The Dirichlet problem with nonhomogeneous energy estimate in a wedge. Let $\mathfrak{Q}$ be the set of functions in $C^{\infty}(\overline{\mathbb{D}} \backslash M)$ with finite norm

$$
\left(\left\|u ; H^{1}(\mathbb{D})\right\|^{2}+\left\|\left.u\right|_{\partial \mathbb{D}} ; H^{1}(\partial \mathbb{D})\right\|^{2}+\left\|\partial_{\nu} u ; L_{2}(\partial \mathbb{D})\right\|^{2}\right)^{1 / 2},
$$

and let $\mathbf{H}$ be the completion of $\mathfrak{Q}$ in the norm (74). We introduce the operator

$$
v \mapsto \mathcal{A}(\tau) v:=\left\{\mathcal{L}\left(x, D_{x}, \tau\right) v,\left.v\right|_{\partial \mathbb{D}}\right\}
$$

with the domain $\left\{v \in \mathfrak{Q}: \mathcal{L}\left(x, D_{x}, \tau\right) v \in L_{2}(\mathbb{D})\right\}$. We regard $\mathcal{A}(\tau)$ as an unbounded operator from $\mathbf{H}$ to $L_{2}(\mathbb{D}) \times H^{1}(\partial \mathbb{D})$ and show that this operator admits closure. Suppose $v_{n} \rightarrow v$ in $\mathbf{H}$ and $\mathcal{A}(\tau) v_{n} \rightarrow\{f, g\}$ in $L_{2}(\mathbb{D}) \times H^{1}(\partial \mathbb{D})$. We assume that $v=0$; this means, in particular, that $\left.v\right|_{\partial \mathbb{D}}=0$, so that $g=0$. It remains to show that $f=0$. For any $w \in C_{c}^{\infty}(\mathbb{D})$ we have

$$
0=\lim _{n \rightarrow \infty}\left(v_{n}, \mathcal{L} w\right)_{\mathbb{D}}=\lim _{n \rightarrow \infty}\left(\mathcal{L} v_{n}, w\right)_{\mathbb{D}}=(f, w)_{\mathbb{D}} .
$$

Consequently, $f=0$. In what follows we denote by $\mathcal{A}(\tau)$ and $(D \mathcal{A}(\tau))$ the closure of the operator and its domain.

Consider the boundary value problem

$$
\begin{aligned}
\mathcal{L}\left(x, D_{x}, \tau\right) v(x) & =f(x), & & x \in \mathbb{D}, \\
v(x) & =g(x), & & x \in \partial \mathbb{D},
\end{aligned}
$$

where $f \in L_{2}(\mathbb{D})$ and $g \in H^{1}(\partial \mathbb{D})$.

Definition 4.2.1. A solution of the equation $\mathcal{A}(\tau) v=\{f, g\}$ is called a strong solution of problem (75), (76). 
We need some preparation for proving the existence and uniqueness of a strong solution. Let $x_{0}=\left(0, z_{0}\right)$ be some point of the edge $M$. In the domain $\Omega=\mathbb{K} \cap \mathcal{S}^{n-1}$, we define the operator pencil $\mathbb{C} \ni \lambda \mapsto \mathfrak{A}_{D}\left(z_{0}, \lambda\right):=r^{2-i \lambda} \mathcal{L}\left(x_{0}, D_{y}, 0\right) r^{i \lambda}$. The map

$$
\mathfrak{A}_{D}\left(z_{0}, \lambda\right): \stackrel{\circ}{H}^{1}(\Omega) \cap H^{2}(\Omega) \mapsto L_{2}(\Omega)
$$

is an isomorphism for all $\lambda \in \mathbb{C}$ except for the normal eigenvalues. Here $H^{s}(\Omega)$ is the usual Sobolev space, and $\stackrel{\circ}{H}^{1}(\Omega)$ is the completion of $\mathcal{C}_{c}^{\infty}(\Omega)$ with respect to the norm of $H^{1}(\Omega)$. Every strip $\{\lambda \in \mathbb{C}:|\operatorname{Im} \lambda|<$ const $\}$ contains at most finitely many eigenvalues of the pencil $\mathfrak{A}_{D}$.

We suppose that the wedge $\mathbb{D}=\mathbb{K} \times \mathbb{R}^{m-n}$ is admissible in the sense of Definition 3.1.1 Then the strip $\{\lambda \in \mathbb{C}: n-3 \leq 2 \operatorname{Im} \lambda \leq n-2\}$ is free of the spectrum of the pencil $\mathfrak{A}_{D}(z, 0)$ for all $z \in M$ (see [30]). We recall that the coefficients of $\mathcal{L}\left(x, D_{x}, D_{y}\right)$ are constant outside of some compact set in $\overline{\mathbb{D}}$. Therefore, the strip $\{\lambda \in \mathbb{C}: n-3 \leq$ $2 \operatorname{Im} \lambda \leq n-2\}$ is at a positive distance from the spectrum of the pencil. In the spaces $H^{1}(\mathbb{D})$ and $H^{1}(\partial \mathbb{D})$, we introduce the following norms depending on a parameter $p>0$ :

$$
\begin{aligned}
\left\|u ; H^{1}(\mathbb{D}, p)\right\|^{2} & =\left\|u ; H^{1}(\mathbb{D})\right\|^{2}+p^{2}\left\|u ; L_{2}(\mathbb{D})\right\|^{2}, \\
\left\|u ; H^{1}(\partial \mathbb{D}, p)\right\|^{2} & =\left\|u ; H^{1}(\partial \mathbb{D})\right\|^{2}+p^{2}\left\|u ; L_{2}(\partial \mathbb{D})\right\|^{2} .
\end{aligned}
$$

Theorem 4.2.2. Suppose that the wedge $\mathbb{D}$ is admissible, $\{f, g\} \in L_{2}(\mathbb{D}) \times H^{1}(\partial \mathbb{D})$, and $\tau=\sigma-i \gamma$ with $\sigma \in \mathbb{R}$ and $\gamma>0$. Then there exists a unique strong solution $v$ of problem (75), (76). We have

$$
\gamma^{2}\left\|v ; H^{1}(\mathbb{D},|\tau|)\right\|^{2}+\gamma\left\|\partial_{\nu} v ; L_{2}(\partial \mathbb{D})\right\|^{2} \leq c\left(\left\|f ; L_{2}(\mathbb{D})\right\|^{2}+\gamma\left\|g ; H^{1}(\partial \mathbb{D},|\tau|)\right\|^{2}\right)
$$

with constant $c$ independent of $\tau$.

Proof. A strong solution of problem (75), (776) with homogeneous boundary condition is also a strong solution of problem (24), (25) (in the sense of Definition 4.1.1). Therefore, the uniqueness of a strong solution of (75), (76) follows from Theorem 4.1.2.

We check existence. First, consider problem (75), (76) with $g=\left.G\right|_{\partial \mathbb{D}}$, where $G \in$ $C_{c}^{\infty}(\overline{\mathbb{D}})$. Putting $u=v-G$, we obtain

$$
\begin{aligned}
\mathcal{L}\left(x, D_{x}, \tau\right) u(x) & =f(x)-\mathcal{L}\left(x, D_{x}, \tau\right) G(x), & & x \in \mathbb{D}, \\
u(x) & =0, & & x \in \partial \mathbb{D} .
\end{aligned}
$$

Let $h_{n} \in C_{c}^{\infty}(\mathbb{D})$ and $h_{n} \rightarrow f-\mathcal{L} G$ in $L_{2}(\mathbb{D})$. The weak solution $u_{n}$ of the problem

$$
\begin{aligned}
\mathcal{L}\left(x, D_{x}, \tau\right) u_{n}(x) & =h_{n}(x), & & x \in \mathbb{D}, \\
u_{n}(x) & =0, & & x \in \partial \mathbb{D},
\end{aligned}
$$

satisfies (51), (52). Then the function $u_{n}+G$ also satisfies those requirements and solves the equations

$$
\begin{aligned}
\mathcal{L}\left(x, D_{x}, \tau\right) u_{n}(x) & =f_{n}(x), & & x \in \mathbb{D}, \\
u_{n}(x) & =g(x), & & x \in \partial \mathbb{D},
\end{aligned}
$$

with $f_{n}(x)=h_{n}(x)+\mathcal{L}\left(x, D_{x}, \tau\right) G(x)$. Therefore, by Proposition 3.1.2, we have

$$
\begin{aligned}
\gamma^{2} \int_{\mathbb{D}} & \left(\left|\nabla_{x} u_{n}(x)\right|^{2}+|\tau|^{2}\left|u_{n}(x)\right|^{2}\right) \mathrm{d} x+\gamma \int_{\partial \mathbb{D}}\left|\partial_{\nu} u_{n}(x)\right|^{2} \mathrm{~d} s \\
& \leq c\left\{\int_{\mathbb{D}}\left|f_{n}(x)\right|^{2} \mathrm{~d} x+\gamma \int_{\partial \mathbb{D}}\left(|\tau|^{2}|g(x)|^{2}+\left|\nabla_{s} g(x)\right|^{2}\right) \mathrm{d} s\right\} .
\end{aligned}
$$

Since $f_{n} \rightarrow f$ in $L_{2}(\mathbb{D})$, the passage to the limit in (80) provides estimate (79) together with the second assertion of the theorem for $f \in L_{2}(\mathbb{D})$ and $g=\left.G\right|_{\partial \mathbb{D}}$, where $G \in C_{c}^{\infty}(\overline{\mathbb{D}})$. 
Now assume that $f \in L_{2}(\mathbb{D})$ and $g \in H^{1}(\partial \mathbb{D})$, and let $g_{n} \rightarrow g$ in $H^{1}(\partial \mathbb{D})$ with $g_{n}=\left.G_{n}\right|_{\partial \mathbb{D}}$, where $G_{n} \in C_{c}^{\infty}(\overline{\mathbb{D}})$. The above argument implies the existence of a strong solution $v_{n}$ of problem (75), (76) with the right-hand side $\left\{f, g_{n}\right\}$. This solution satisfies an inequality of the form (79). The passage to the limit in this inequality completes the proof.

Now, we look at the time-dependent problem

$$
\begin{aligned}
& \mathcal{L}\left(x, D_{x}, D_{t}\right) V(x, t)=F(x, t), \quad x \in \mathbb{D}, \quad-\infty<t<+\infty, \\
& V(x, t)=G(x, t), \quad x \in \partial \mathbb{D}, \quad-\infty<t<+\infty .
\end{aligned}
$$

Using (79) and the inverse Fourier transform

$$
u(x, t)=\frac{1}{\sqrt{2} \pi} \int_{\operatorname{Im} \tau=\gamma} \exp (i t \tau) \hat{u}(x, \tau) \mathrm{d} \tau,
$$

for the solution of problem (81), (82) we obtain the inequality

$$
\begin{aligned}
& \gamma^{2} \int_{\mathbb{D}} \int_{-\infty}^{+\infty} e^{-2 \gamma t}\left(|V(x, t)|^{2}+\left|\nabla_{x, t} V(x, t)\right|^{2}\right) \mathrm{d} x \mathrm{~d} t \\
& +\gamma \int_{\partial \mathbb{D}} \int_{-\infty}^{+\infty} e^{-2 \gamma t}\left|\partial_{\nu} V(x, t)\right|^{2} \mathrm{~d} S_{x} \mathrm{~d} t \\
& \quad \leq c\left(\int_{\mathbb{D}} \int_{-\infty}^{+\infty} e^{-2 \gamma t}|F(x, t)|^{2} \mathrm{~d} x \mathrm{~d} t\right. \\
& \left.\quad+\gamma \int_{\partial \mathbb{D}} \int_{-\infty}^{+\infty} e^{-2 \gamma t}\left(|G(x, t)|^{2}+\left|\nabla_{s} G(x, t)\right|^{2}\right) \mathrm{d} S_{x} \mathrm{~d} t\right),
\end{aligned}
$$

where $\nabla_{s}$ is the tangent component of the gradient. Therefore, the trace of $\nabla_{x, t} V$ on $\partial \mathbb{D} \times \mathbb{R}$ makes sense as an element of the space $L_{2, \gamma}(\partial \mathbb{D} \times \mathbb{R})$ of functions square integrable with the weight $t \mapsto e^{-2 \gamma t}$. This allows us to define the deformation and stress tensors on $\partial \mathbb{D} \times \mathbb{R}$; we could not do that on the basis of Theorem 4.1.2 (even for the homogeneous boundary condition) without some additional requirements on the right-hand side of (69).

4.3. The Neumann problem in a wedge. Consider the problem

$$
\begin{array}{rlrl}
\mathcal{L}\left(x, D_{x}, \tau\right) v(x) & =f(x), & & x \in \mathbb{D}, \\
\mathcal{B}\left(x, D_{x}\right) v(x) & =g(x), \quad & x \in \partial \mathbb{D} \backslash M .
\end{array}
$$

For example, we analyze the case where $\operatorname{dim} \mathbb{K}=n>2$. We introduce the operator

$$
\mathcal{A}(\tau) v=\left\{\mathcal{L}\left(x, D_{x}, \tau\right) v,\left.\mathcal{B}\left(x, D_{x}\right) v\right|_{\partial \mathbb{D}}\right\}
$$

with the domain

$$
\left\{v \in \mathcal{C}^{\infty}(\overline{\mathbb{D}} \backslash M) \cap H^{1}(\mathbb{D}): \mathcal{B}\left(x, D_{x}\right) v \in H_{0}^{-1 / 2}(\partial \mathbb{D}), \mathcal{L}\left(x, D_{x}, \tau\right) v \in L_{2}(\mathbb{D})\right\} .
$$

We view $\mathcal{A}(\tau)$ as an unbounded operator from $H^{1}(\mathbb{D})$ to $L_{2}(\mathbb{D}) \times H_{0}^{-1 / 2}(\partial \mathbb{D})$. This operator admits closure. Indeed, suppose $v_{n} \rightarrow v$ in $H^{1}(\mathbb{D})$ and $\left\{\mathcal{L} v_{n}, \mathcal{B} v_{n}\right\} \rightarrow\{f, g\}$ in $L_{2}(\mathbb{D}) \times H_{0}^{-1 / 2}(\partial \mathbb{D})$. We assume that $v=0$. Then for any $w \in C_{c}^{\infty}(\mathbb{D})$ we have $(f, w)=\lim \left(\mathcal{L}(\tau) v_{n}, w\right)=\lim \left(v_{n}, \mathcal{L}(\bar{\tau}) w\right)=0$, whence $f=0$. Choose a function $w$ such that $\mathcal{B} w=0$ on $\partial \mathbb{D}$ and $\left.w\right|_{\partial \mathbb{D}}$ is an arbitrary function in $C_{c}^{\infty}(\partial \mathbb{D} \backslash M)$ given beforehand. Letting $n \rightarrow \infty$ in the Green formula

$$
\left(\mathcal{L}(\tau) v_{n}, w\right)_{\mathbb{D}}+\left(\mathcal{B} v_{n}, w\right)_{\partial \mathbb{D}}=\left(v_{n}, \mathcal{L}(\bar{\tau}) w\right)_{\mathbb{D}}+\left(v_{n}, \mathcal{B} w\right)_{\partial \mathbb{D}}
$$

we obtain $(\mathcal{B} v, w)_{\partial \mathbb{D}}=0$. Hence, $g=0$. In what follows, $\mathcal{A}(\tau)$ and $D(\mathcal{A}(\tau))$ denote the closed operator and its domain. 
A solution of the equation $\mathcal{A}(\tau) v=\{f, g\}$ is called a strong solution of problem (83), (84).

As in the case of the Dirichlet problem, we also need a weak solution. By definition, a function $v \in H^{1}(\mathbb{D})$ is a weak solution of problem (83), (84) if $B(v, w)=$ $(f, w)_{\mathbb{D}}-i(g, w)_{\partial \mathbb{D}}$ for all $w$ in $H^{1}(\mathbb{D})$; here $B$ denotes the same form as in $(72)$. The functional $l(w)=(f, w)_{\mathbb{D}}-i(g, w)_{\partial \mathbb{D}}$ is bounded, i.e., $|l(w)| \leq c\left\|w ; H_{1}(\mathbb{D})\right\| \cdot\left(\left\|f ; L_{2}(\mathbb{D})\right\|+\right.$ $\left.\left\|g ; H_{0}^{-1 / 2}(\partial \mathbb{D})\right\|\right)$. The existence and uniqueness of a weak solution follow from the LaxMilgram-Vishik lemma and Proposition 4.1.3.

Theorem 4.3.1. For any $\{f, g\} \in L_{2}(\mathbb{D}) \times H_{0}^{-1 / 2}(\partial \mathbb{D})$ and any $\tau=\sigma-i \gamma$ with $\sigma \in \mathbb{R}$ and $\gamma>0$, there exists a unique strong solution $v$ of problem (83), (84). The estimate

$$
\gamma^{2}\left\|v ; H^{1}(\mathbb{D},|\tau|)\right\|^{2} \leq c\left(\left\|f ; L_{2}(\mathbb{D})\right\|^{2}+|\tau|^{2}\left\|g ; H_{0}^{-1 / 2}(\partial \mathbb{D})\right\|^{2}\right)
$$

is true with constant $c$ independent of $\tau$.

Proof. Inequality (61) remains valid for the elements of $D(\mathcal{A}(\tau))$. Consequently, the kernel of $\mathcal{A}(\tau)$ is trivial and the range of $\mathcal{A}(\tau)$ is closed. Also, we have $C_{c}^{\infty}(\mathbb{D}) \times C_{c}^{\infty}(\partial \mathbb{D} \backslash$ $M) \subset \mathcal{R} \mathcal{A}(\tau)$, because a weak solution belongs to $D(\mathcal{A}(\tau))$. Since the range $\mathcal{R} \mathcal{A}(\tau)$ is closed, we obtain $\mathcal{R} \mathcal{A}(\tau)=L_{2}(\mathbb{D}) \times H_{0}^{-1 / 2}(\partial \mathbb{D})$.

\section{§5. WEIGHTED a priori ESTIMATES FOR SOLUTIONS OF BOUNDARY VALUE PROBLEMS WITH PARAMETER IN A WEDGE}

Estimates in spaces with weighted norms make it possible to get asymptotic formulas for solutions near the edge. The proof of such estimates consists of three stages. At the first stage, we establish a weighted "hyperbolic" inequality far from the edge. The second stage is devoted to an "elliptic" estimate in a neighborhood of the edge. At the final stage, the "intermediate" zone is treated with the help of an energy estimate.

Assume that $s$ is a nonnegative integer and $\beta \in \mathbb{R}$. The space $H_{\beta}^{s}(\mathbb{D})$ is the completion of $\mathcal{C}_{c}^{\infty}(\overline{\mathbb{D}} \backslash M)$ in the norm

$$
\left\|u ; H_{\beta}^{s}(\mathbb{D})\right\|=\left(\sum_{|\alpha| \leq s} \int_{\mathbb{D}} r^{2(\beta+|\alpha|-s)}\left|D_{x}^{\alpha} u(x)\right|^{2} \mathrm{~d} x\right)^{1 / 2},
$$

where $x=(y, z) \in \mathbb{D}=\mathbb{K} \times \mathbb{R}^{m-n}, r=|y|$. The space of traces on $\partial \mathbb{D}$ of the functions in $H_{\beta}^{s}(\mathbb{D})$ with $s \geq 1$ is denoted by $H_{\beta}^{s-1 / 2}(\partial \mathbb{D})$. The space $H_{\beta}^{s}(\mathbb{D}, p)$ with positive parameter $p$ is endowed with the norm

$$
\left\|u ; H_{\beta}^{s}(\mathbb{D}, p)\right\|=\left(\sum_{k=0}^{s} p^{2 k}\left\|u ; H_{\beta}^{s-k}(\mathbb{D})\right\|^{2}\right)^{1 / 2} .
$$

Finally, for $s \geq 1$ we denote by $H_{\beta}^{s-1 / 2}(\partial \mathbb{D}, p)$ the space of traces on $\partial \mathbb{D}$ of the functions of class $H_{\beta}^{s}(\mathbb{D}, p)$.

5.1. Estimates of solutions with Dirichlet boundary condition. In this subsection, we suppose that the coefficients of $\mathcal{L}$ are constant, so that

$$
\mathcal{L}\left(D_{x}, D_{t}\right) u=\partial_{t}^{2}+A\left(D_{x}\right) u,
$$

where $A\left(D_{x}\right)=A\left(D_{y}, D_{z}\right)=D_{k} A^{k l} D_{l}, A^{k l}=\left\|a_{i j}^{k l}\right\|_{i, j=1}^{m}$, and the $a_{i j}^{k l}$ are some constants. Recall that the operator pencil $\mathbb{C} \ni \lambda \mapsto \mathfrak{A}_{D}(\lambda) v:=\left\{r^{2-i \lambda} A\left(D_{y}, 0\right) r^{i \lambda} v,\left.v\right|_{\partial \Omega}\right\}$ in the domain $\Omega=\mathbb{K} \cap S^{n-1}$ yields an isomorphism

$$
\mathfrak{A}_{D}(\lambda): H^{2}(\Omega) \rightarrow L_{2}(\Omega) \times H^{3 / 2}(\partial \Omega)
$$


for all $\lambda \in \mathbb{C}$ except for the normal eigenvalues. Every strip $\{\lambda \in \mathbb{C}:|\operatorname{Im} \lambda|<$ const $\}$ contains at most finitely many eigenvalues of the pencil $\mathfrak{A}_{D}$.

The next proposition presents a weighted estimate of solutions with Dirichlet boundary condition, which is the basic result of the subsection. The proof employs a homogeneous (or nonhomogeneous) energy inequality. Therefore the corresponding estimate is valid for an arbitrary (or, respectively, admissible) wedge (see Definition 3.1.1).

For $\beta \leq 1$, we introduce the set

$$
\left\{u \in H_{\beta}^{2}(\mathbb{D}, 1) \cap H^{1}(\mathbb{D}): \mathcal{L}\left(D_{x}, 0\right) u \in L_{2}(\mathbb{D}),\left.u\right|_{\partial \mathbb{D}} \in H_{\beta}^{1}(\partial \mathbb{D}, 1) \cap H^{1}(\partial \mathbb{D})\right\} .
$$

It can be shown that the relation $\left.u\right|_{\partial \mathbb{D}} \in H_{\beta}^{1}(\partial \mathbb{D}, 1)$ follows from the conditions $\left.u\right|_{\partial \mathbb{D}} \in$ $H^{1}(\partial \mathbb{D})$ and $u \in H_{\beta}^{2}(\mathbb{D}, 1) \cap H^{1}(\mathbb{D})$.

Proposition 5.1.1. Assume that the line $\operatorname{Im} \lambda=\beta+n / 2-2$ is free of the spectrum of the pencil $\mathfrak{A}_{D}$, and $\beta \leq 1$. Then:

1) for the elements of the set (87) that satisfy $\left.u\right|_{\partial \mathbb{D}}=0$, we have

$$
\begin{aligned}
& \left\|\chi_{\tau} u ; H_{\beta}^{2}(\mathbb{D},|\tau|)\right\|^{2}+\gamma^{2}\left\|u ; H_{\beta}^{1}(\mathbb{D},|\tau|)\right\|^{2} \\
& \quad \leq c\left\{\left\|\mathcal{L}\left(D_{x}, \tau\right) u ; H_{\beta}^{0}(\mathbb{D})\right\|^{2}+\left(|\tau|^{2(1-\beta)} / \gamma^{2}\right)\left\|\mathcal{L}\left(D_{x}, \tau\right) u ; L_{2}(\mathbb{D})\right\|^{2}\right\},
\end{aligned}
$$

where $\chi_{\tau}(x)=\chi(|\tau| y), x=(y, z)$, and $\chi \in \mathcal{C}_{c}^{\infty}(\overline{\mathbb{K}})$ is a cut-off function equal to 1 near the vertex of $\mathbb{K}$, while $\mathbb{D}=\mathbb{K} \times \mathbb{R}^{m-n}$ and $\mathbb{K}$ is an arbitrary open cone smooth outside the vertex;

2) in an admissible wedge $\mathbb{D}$, for the elements of the set (87) we have

$$
\begin{aligned}
& \left\|\chi_{\tau} u ; H_{\beta}^{2}(\mathbb{D},|\tau|)\right\|^{2}+\gamma^{2}\left\|u ; H_{\beta}^{1}(\mathbb{D},|\tau|)\right\|^{2}+\gamma\left\|\partial_{\nu} u ; H_{\beta}^{0}(\partial \mathbb{D})\right\|^{2} \\
& \leq c\left\{\left\|\mathcal{L}\left(D_{x}, \tau\right) u ; H_{\beta}^{0}(\mathbb{D})\right\|^{2}+\left\|\chi_{\tau} u ; H_{\beta}^{3 / 2}(\partial \mathbb{D})\right\|^{2}+\gamma\left\|u ; H_{\beta}^{1}(\partial \mathbb{D},|\tau|)\right\|^{2}\right. \\
& \quad+\left(|\tau|^{2(1-\beta)} / \gamma^{2}\right)\left(\left\|\mathcal{L}\left(D_{x}, \tau\right) u ; L_{2}(\mathbb{D})\right\|^{2}+\gamma\left\|u ; H^{1}\left((\partial \mathbb{D},|\tau|)^{2}\right)\right\|\right\},
\end{aligned}
$$

where $\chi_{\tau}$ is the same function as in (88).

The constants $c$ in (88) and (89) are independent of $u$ and of $\tau=\sigma-i \gamma$ with $\sigma \in \mathbb{R}$ and $\gamma>0$.

Proof. We verify only the second part of the proposition; the argument for the first part is simpler.

An estimate far from the edge. We set $x=(y, z), r=|y|$ and denote by $\kappa$ and $\psi$ some cut-off functions of class $\mathcal{C}_{c}^{\infty}(\overline{\mathbb{K}})$ such that $\operatorname{supp} \kappa \in\{y \in \mathbb{K}: 1 / 2<r<2\}$, $\operatorname{supp} \psi \in\{y \in \mathbb{K}: 1 / 4<r<4\}$, and $\kappa \psi=\kappa$. We extend $\psi$ and $\kappa$ to $\overline{\mathbb{D}}$ assuming that the extended functions are independent of $z$, and keeping the same notation. Let $v$ be an arbitrary element of the set (87). We put $v^{\varepsilon}(x)=v(x / \varepsilon)$, substitute $\kappa v^{\varepsilon}$ in (53), and take $\tau /|\tau| \varepsilon$ instead of $\tau$, arriving at

$$
\begin{gathered}
(\gamma /|\tau| \varepsilon)^{2}\left(\left\|\nabla_{x}\left(\kappa v^{\varepsilon}\right) ; L_{2}(\mathbb{D})\right\|^{2}+\left(1 / \varepsilon^{2}\right)\left\|\kappa v^{\varepsilon} ; L_{2}(\mathbb{D})\right\|^{2}\right)+(\gamma /|\tau| \varepsilon)\left\|\partial_{\nu}\left(\kappa v^{\varepsilon}\right) ; H_{\beta}^{0}(\partial \mathbb{D})\right\|^{2} \\
\leq c\left\{\left\|\kappa \mathcal{L}\left(D_{x}, \tau /|\tau| \varepsilon\right) v^{\varepsilon} ; L_{2}(\mathbb{D})\right\|^{2}+\left(\gamma /|\tau| \varepsilon^{3}\right)\left\|\kappa v^{\varepsilon} ; L_{2}(\partial \mathbb{D})\right\|^{2}\right. \\
\left.+\left\|\psi v^{\varepsilon} ; H^{1}(\mathbb{D})\right\|^{2}+(\gamma /|\tau| \varepsilon)\left\|\nabla_{s}\left(\kappa v^{\varepsilon}\right) ; L_{2}(\partial \mathbb{D})\right\|^{2}\right\} .
\end{gathered}
$$

After the change of variables $x \mapsto \xi=x / \varepsilon$, this inequality takes the form

$$
\begin{array}{r}
(\gamma /|\tau|)^{2}\left(\left\|\nabla_{\xi}\left(\kappa_{\varepsilon} v\right) ; L_{2}(\mathbb{D})\right\|^{2}+\left\|\kappa_{\varepsilon} v ; L_{2}(\mathbb{D})\right\|^{2}\right)+(\gamma /|\tau|)\left\|\partial_{\nu}\left(\kappa_{\varepsilon} v\right) ; H_{\beta}^{0}(\partial \mathbb{D})\right\|^{2} \\
\leq c\left\{\left\|\kappa_{\varepsilon} \mathcal{L}\left(D_{x}, \theta\right) v ; L_{2}(\mathbb{D})\right\|^{2}+(\gamma /|\tau|)\left\|\kappa_{\varepsilon} v ; L_{2}(\partial \mathbb{D})\right\|^{2}+\varepsilon^{4}\left\|\psi_{\varepsilon} v ; L_{2}(\mathbb{D})\right\|^{2}\right. \\
\left.+\varepsilon^{2}\left\|\nabla_{\xi}\left(\psi_{\varepsilon} v\right) ; L_{2}(\mathbb{D})\right\|^{2}+(\gamma /|\tau|)\left\|\nabla_{s}\left(\kappa_{\varepsilon} v\right) ; L_{2}(\partial \mathbb{D})\right\|^{2}\right\}
\end{array}
$$


where $\theta=\tau /|\tau|, \kappa_{\varepsilon}(\xi)=\kappa(\varepsilon \xi)$, etc. We multiply the relation by $\varepsilon^{-2 \beta}$, put $\varepsilon=2^{-j}$, and add all such inequalities for $j=0,1,2, \ldots$ This yields

$$
\begin{aligned}
&(\gamma /|\tau|)^{2}\left(\left\|\nabla_{\xi}\left(\kappa_{\infty} v\right) ; H_{\beta}^{0}(\mathbb{D})\right\|^{2}+\left\|\kappa_{\infty} v ; H_{\beta}^{0}(\mathbb{D})\right\|^{2}\right)+(\gamma /|\tau|)\left\|\partial_{\nu}\left(\kappa_{\infty} v\right) ; H_{\beta}^{0}(\partial \mathbb{D})\right\|^{2} \leq c\left\{\left\|\kappa_{\infty} \mathcal{L}\left(D_{\xi}, \theta\right) v ; H_{\beta}^{0}(\mathbb{D})\right\|^{2}+(\gamma /|\tau|)\left\|\kappa_{\infty} v ; H_{\beta}^{0}(\partial \mathbb{D})\right\|^{2}\right. \\
&\left.\quad+\left\|\psi_{\infty} v ; H_{\beta-1}^{1}(\mathbb{D})\right\|^{2}+(\gamma /|\tau|)\left\|\nabla_{s}\left(\psi_{\infty} v\right) ; H_{\beta}^{0}(\partial \mathbb{D})\right\|^{2}\right\}
\end{aligned}
$$

where the cut-off functions $\kappa_{\infty}$ and $\psi_{\infty}$ are in $\mathcal{C}^{\infty}(\overline{\mathbb{K}})$, are equal to 0 near the vertex of the cone and to 1 in a neighborhood of infinity, and satisfy $\kappa_{\infty} \psi_{\infty}=\kappa_{\infty}$.

An estimate in a neighborhood of the edge. Consider the operator

$$
\mathcal{A}: H_{\beta}^{2}(\mathbb{D}) \rightarrow H_{\beta}^{0}(\mathbb{D}) \times H_{\beta}^{3 / 2}(\partial \mathbb{D})
$$

given by the formula $v \mapsto \mathcal{A} v:=\left\{A\left(D_{\xi}\right) v,\left.v\right|_{\partial \mathbb{D}}\right\}$. It is known (see [17) that if $\beta \leq 1$ and the line $\operatorname{Im} \lambda=\beta+n / 2-2$ is free of the spectrum of the pencil $\mathfrak{A}_{D}$, then $\mathcal{A}$ is a monomorphism and

$$
\left\|v ; H_{\beta}^{2}(\mathbb{D})\right\|^{2} \leq c\left\{\left\|A\left(D_{\xi}\right) v ; H_{\beta}^{0}(\mathbb{D})\right\|^{2}+\left\|v ; H_{\beta}^{3 / 2}(\partial \mathbb{D})\right\|^{2}\right\}
$$

Let $\chi$ and $\psi$ be cut-off functions of class $\mathcal{C}_{c}^{\infty}(\overline{\mathbb{D}})$ equal to 1 near the edge and such that $\chi \psi=\chi$; we also assume that $\chi$ and $\psi$ are independent of the coordinate $z$ of $x=(y, z)$. By (91), we have

$$
\begin{aligned}
& \left\|\chi v ; H_{\beta}^{2}(\mathbb{D}, 1)\right\|^{2} \\
& \quad \leq c\left\{\left\|\chi \mathcal{L}\left(D_{\xi}, \theta\right) v ; H_{\beta}^{0}(\mathbb{D})\right\|^{2}+\left\|\chi v ; H_{\beta}^{3 / 2}(\partial \mathbb{D})\right\|^{2}+\left\|\psi v ; H_{\beta}^{1}(\mathbb{D}, 1)\right\|^{2}\right\} .
\end{aligned}
$$

The global estimate. We add (90) and (92). The definition of the norms involved shows that $\kappa_{\infty}$ on the left-hand side can be replaced with 1 . Thus,

$$
\begin{aligned}
& \left\|\chi v ; H_{\beta}^{2}(\mathbb{D}, 1)\right\|^{2}+(\gamma /|\tau|)^{2}\left\|v ; H_{\beta}^{1}(\mathbb{D}, 1)\right\|^{2}+(\gamma /|\tau|)\left\|\partial_{\nu} v ; H_{\beta}^{0}(\partial \mathbb{D})\right\|^{2} \\
& \leq c\left\{\left\|\mathcal{L}\left(D_{\xi}, \theta\right) v ; H_{\beta}^{0}(\mathbb{D})\right\|^{2}+\left\|\chi v ; H_{\beta}^{3 / 2}(\partial \mathbb{D})\right\|^{2}\right. \\
& \quad+(\gamma /|\tau|)\left\|\kappa_{\infty} v ; H_{\beta}^{0}(\partial \mathbb{D})\right\|^{2}+(\gamma /|\tau|)\left\|\nabla_{s}\left(\kappa_{\infty} v\right) ; H_{\beta}^{0}(\partial \mathbb{D})\right\|^{2} \\
& \left.\quad+\left\|\psi_{\infty} v ; H_{\beta-1}^{1}(\mathbb{D})\right\|^{2}+\left\|\psi v ; H_{\beta}^{1}(\mathbb{D}, 1)\right\|^{2}\right\} .
\end{aligned}
$$

Here we make the change of variables $\xi \mapsto x=\xi /|\tau|$ and put $u(x)=v(|\tau| x)$, obtaining

$$
\begin{gathered}
\left\|\chi_{\tau} u ; H_{\beta}^{2}(\mathbb{D},|\tau|)\right\|^{2}+\gamma^{2}\left\|u ; H_{\beta}^{1}(\mathbb{D},|\tau|)\right\|^{2}+\gamma\left\|\partial_{\nu} u ; H_{\beta}^{0}(\partial \mathbb{D})\right\|^{2} \\
\leq c\left\{\left\|\mathcal{L}\left(D_{x}, \tau\right) u ; H_{\beta}^{0}(\mathbb{D})\right\|^{2}+\left\|\chi_{\tau} u ; H_{\beta}^{3 / 2}(\partial \mathbb{D})\right\|^{2}\right. \\
+\gamma\left\|\psi_{\infty, \tau} u ; H_{\beta}^{1}(\partial \mathbb{D},|\tau|)\right\|^{2}+\left\|\psi_{\infty, \tau} u ; H_{\beta-1}^{1}(\mathbb{D})\right\|^{2} \\
\left.+|\tau|^{2}\left\|\psi_{\tau} u ; H_{\beta}^{1}(\mathbb{D},|\tau|)\right\|^{2}\right\}
\end{gathered}
$$


where $\chi_{\tau}(y)=\chi(|\tau| y), \psi_{\tau}(y)=\psi(|\tau| y)$, and $\psi_{\infty, \tau}(y)=\psi_{\infty}(|\tau| y)$. We estimate the last two terms on the right. We have

$$
\begin{aligned}
& |\tau|^{2}\left\|\psi_{\tau} u ; H_{\beta}^{1}(\mathbb{D},|\tau|)\right\|^{2} \\
& \leq|\tau|^{2}\left(\int_{r<c /|\tau|} r^{2 \beta}\left(|\nabla u|^{2}+|u|^{2} / r^{2}\right) \mathrm{d} x+|\tau|^{2} \int_{r<c /|\tau|} r^{2 \beta}|u|^{2} \mathrm{~d} x\right) \\
& \leq|\tau|^{2}\left(\int_{r<\varepsilon /|\tau|} r^{2 \beta}\left(|\nabla u|^{2}+|u|^{2} / r^{2}+|\tau|^{2}|u|^{2}\right) \mathrm{d} x\right. \\
& \left.\quad+\int_{\varepsilon /|\tau|<r<c /|\tau|} r^{2 \beta}\left(|\nabla u|^{2}+|u|^{2} / r^{2}+|\tau|^{2}|u|^{2}\right) \mathrm{d} x\right) \\
& \leq c \varepsilon^{2} \int_{r<\varepsilon /|\tau|} r^{2(\beta-1)}\left(|\nabla u|^{2}+|u|^{2} / r^{2}+|\tau|^{2}|u|^{2}\right) \mathrm{d} x+\left\|\psi_{\infty, \tau} u ; H_{\beta-1}^{1}(\mathbb{D})\right\|^{2} .
\end{aligned}
$$

Therefore,

$$
|\tau|^{2}\left\|\psi_{\tau} u ; H_{\beta}^{1}(\mathbb{D},|\tau|)\right\|^{2} \leq \varepsilon^{2}\left\|\chi_{\tau} u ; H_{\beta}^{2}(\mathbb{D},|\tau|)\right\|^{2}+\left\|\psi_{\infty, \tau} u ; H_{\beta-1}^{1}(\mathbb{D})\right\|^{2} .
$$

This and (93) lead to the inequality

$$
\begin{aligned}
\| \chi_{\tau} u ; & H_{\beta}^{2}(\mathbb{D},|\tau|)\left\|^{2}+\gamma^{2}\right\| u ; H_{\beta}^{1}(\mathbb{D},|\tau|)\left\|^{2}+\gamma\right\| \partial_{\nu} u ; H_{\beta}^{0}(\partial \mathbb{D}) \|^{2} \\
\leq c\{ & \left\|\mathcal{L}\left(D_{x}, \tau\right) u ; H_{\beta}^{0}(\mathbb{D})\right\|^{2}+\left\|\chi_{\tau} u ; H_{\beta}^{3 / 2}(\partial \mathbb{D})\right\|^{2} \\
& \left.\quad+\gamma\left\|\psi_{\infty, \tau} u ; H_{\beta}^{1}(\partial \mathbb{D},|\tau|)\right\|^{2}+\left\|\psi_{\infty, \tau} u ; H_{\beta-1}^{1}(\mathbb{D})\right\|^{2} \varepsilon^{2}\left\|\chi_{\tau} u ; H_{\beta}^{2}(\mathbb{D},|\tau|)\right\|^{2}\right\} .
\end{aligned}
$$

Since the left-hand side contains $\left\|\chi_{\tau} u ; H_{\beta}^{2}(\mathbb{D},|\tau|)\right\|^{2}$, by increasing the constant $c$ we can remove $\varepsilon^{2}\left\|\chi_{\tau} u ; H_{\beta}^{2}(\mathbb{D},|\tau|)\right\|^{2}$ from the right-hand side for small $\varepsilon$. Finally, for $\beta \leq 1$ we obtain

$$
\begin{gathered}
\left\|\psi_{\infty, \tau} u ; H_{\beta-1}^{1}(\mathbb{D})\right\|^{2} \leq \int_{c /|\tau|<r} r^{2(\beta-1)}\left(|\nabla u|^{2}+|u|^{2} / r^{2}\right) \mathrm{d} x \\
\leq c|\tau|^{2(1-\beta)}\left\{\left\|\nabla u ; L_{2}(\mathbb{D})\right\|^{2}+|\tau|^{2}\left\|u ; L_{2}(\mathbb{D})\right\|^{2}\right\},
\end{gathered}
$$

which can be majorized with the help of (53).

5.2. Estimate of solutions with Neumann boundary condition for $\operatorname{dim} \mathbb{K}>2$. In contrast to the Dirichlet problem, the energy estimate for solutions of the Neumann problem with nonhomogeneous boundary condition was proved without the additional assumption about the admissibility of the wedge (see Definition 3.1.1). Therefore, a weighted estimate for solutions of the Neumann problem can be obtained in the wedge $\mathbb{D}=\mathbb{K} \times \mathbb{R}^{m-n}$, where $\mathbb{K}$ is an arbitrary open cone in $\mathbb{R}^{n}$ smooth outside the vertex. We treat the cases where $\operatorname{dim} \mathbb{K}=2$ and where $\operatorname{dim} \mathbb{K}>2$ separately. The reason is that the "elliptic" part of the estimate involves weighted norms defined differently for the cases mentioned (a "homogeneous" norm for $\operatorname{dim} \mathbb{K}>2$ and a "nonhomogeneous" norm for $\operatorname{dim} \mathbb{K}=2)$.

We consider the operator of the Neumann problem in $\mathbb{D}$,

$$
v \mapsto\left\{\mathcal{L}\left(D_{x}, \tau\right) v,\left.\mathcal{B}\left(\omega, D_{x}\right) v\right|_{\partial \mathbb{D}}\right\} ;
$$

here $x=(y, z)$, the coefficients of $\mathcal{L}$ are constant, and those of $\mathcal{B}$ depend only on $\omega=y /|y|$ (because the normal to $\partial \mathbb{K} \backslash \mathcal{O}$ depends only on $\omega$ ). In the domain $\Omega=\mathbb{K} \cap \mathcal{S}^{n-1}$ we define the operator pencil

$$
\mathbb{C} \ni \lambda \mapsto \mathfrak{A}_{N}(\lambda):=\left\{r^{2-i \lambda} A\left(D_{y}, 0\right) r^{i \lambda}, r^{1-i \lambda} \mathcal{B}\left(\omega, D_{y}, 0\right) r^{i \lambda}\right\} .
$$


The map $\mathfrak{A}_{N}(\lambda): H^{2}(\Omega) \mapsto L_{2}(\Omega) \times H^{1 / 2}(\partial \Omega)$ is an isomorphism for all $\lambda \in \mathbb{C}$ except for the normal eigenvalues. Every strip $\{\lambda \in \mathbb{C}:|\operatorname{Im} \lambda|<$ const $\}$ contains at most finitely many points of the spectrum of the pencil $\mathfrak{A}$.

We introduce the set

$$
\begin{aligned}
& \left\{u \in H_{\beta}^{2}(\mathbb{D}, 1) \cap H^{1}(\mathbb{D}):\right. \\
& \left.\quad \mathcal{L}\left(D_{x}, 0\right) u \in L_{2}(\mathbb{D}), \mathcal{B}\left(\omega, D_{x}\right) u \in H_{\beta+1 / 2}^{0}(\partial \mathbb{D}) \cap H_{0}^{-1 / 2}(\partial \mathbb{D})\right\} .
\end{aligned}
$$

Proposition 5.2.1. Assume that $\beta \leq 1, \gamma>0$, and that the line $\operatorname{Im} \lambda=\beta+n / 2-2$ contains no eigenvalues of the pencil $\mathfrak{A}_{N}$. Then for the functions in the set (94) we have

$$
\begin{aligned}
\left\|\chi_{\tau} u ; H_{\beta}^{2}(\mathbb{D},|\tau|)\right\|^{2}+\gamma^{2}\left(\left\|\nabla_{x} u ; H_{\beta}^{0}(\mathbb{D})\right\|^{2}+|\tau|^{2}\left\|u ; H_{\beta}^{0}(\mathbb{D})\right\|^{2}\right) \\
\leq c\left\{\left\|\mathcal{L}\left(D_{x}, \tau\right) u ; H_{\beta}^{0}(\mathbb{D})\right\|^{2}+\left\|\chi_{\tau} \mathcal{B}\left(\omega, D_{x}\right) u ; H_{\beta}^{1 / 2}(\partial \mathbb{D})\right\|^{2}\right. \\
\quad+|\tau|^{2}\left\|\mathcal{B}\left(D_{x}\right) u ; H_{\beta+1 / 2}^{0}(\partial \mathbb{D})\right\|^{2}+\left(|\tau|^{2(3-\beta)} / \gamma^{4}\right)\left(\left\|\mathcal{L}\left(D_{x}, \tau\right) u ; L_{2}(\mathbb{D})\right\|\right. \\
\left.\left.\quad+|\tau|^{2}\left\|\mathcal{B}\left(\omega, D_{x}\right) v ; H_{0}^{-1 / 2}(\partial \mathbb{D})\right\|^{2}\right)\right\}
\end{aligned}
$$

with constant $c$ independent of $\tau=\sigma-i \gamma$, where $\sigma \in \mathbb{R}$ and $\gamma>0$. Here $\chi_{\tau}(y)=\chi(|\tau| y)$, while $\chi \in \mathcal{C}_{c}^{\infty}(\overline{\mathbb{K}})$ is a cut-off function equal to 1 in a neighborhood of the vertex of $\mathbb{K}$; the function $\chi_{\tau}$ is assumed to be extended to $\overline{\mathbb{D}}$ and independent of $z$.

Proof. An estimate far from the edge. Let $\kappa$ and $\psi$ be cut-off functions of class $\mathcal{C}_{c}^{\infty}(\overline{\mathbb{D}})$ such that $\operatorname{supp} \kappa \in\{x=(y, z) \in \mathbb{D}: 1 / 2<|y|<2\}, \operatorname{supp} \psi \in\{x=(y, z) \in \mathbb{D}: 1 / 4<$ $|y|<4\}$, and $\kappa \psi=\kappa$; we assume that $\psi$ and $\kappa$ are independent of $z$. Replacing $v$ in (61) by $\kappa v$, where $v$ is an element of the set (94), we have

$$
\begin{aligned}
& \gamma^{2}\left\|\nabla_{x}(\kappa v) ; L_{2}(\mathbb{D})\right\|^{2}+\gamma^{2}|\tau|^{2}\left\|\kappa v ; L_{2}(\mathbb{D})\right\|^{2} \\
& \leq c\left\{\left\|\kappa \mathcal{L}\left(D_{x}, \tau\right) v ; L_{2}(\mathbb{D})\right\|^{2}+|\tau|^{2}\left\|\kappa \mathcal{B}\left(\omega, D_{x}\right) v ; H_{0}^{-1 / 2}(\partial \mathbb{D})\right\|^{2}\right. \\
& \left.\quad+\left\|\psi v ; H^{1}(\mathbb{D})\right\|^{2}+|\tau|^{2}\left\|\left(\mathcal{B}\left(\omega, D_{x}\right) \kappa\right) v ; H_{0}^{-1 / 2}(\partial \mathbb{D})\right\|^{2}\right\} .
\end{aligned}
$$

Replacing the function $x \mapsto v(x)$ with $x \mapsto v^{\varepsilon}(x)=v(x / \varepsilon)$ and the parameter $\tau$ with the new parameter $\tau /|\tau| \varepsilon$, we obtain

$$
\begin{aligned}
& (\gamma /|\tau| \varepsilon)^{2}\left\|\nabla_{x}\left(\kappa v^{\varepsilon}\right) ; L_{2}(\mathbb{D})\right\|^{2}+\left(\gamma /|\tau| \varepsilon^{2}\right)^{2}\left\|\kappa v^{\varepsilon} ; L_{2}(\mathbb{D})\right\|^{2} \\
& \leq c\left\{\left\|\kappa \mathcal{L}\left(D_{x}, \tau /|\tau| \varepsilon\right) v^{\varepsilon} ; L_{2}(\mathbb{D})\right\|^{2}+(1 / \varepsilon)^{2}\left\|\kappa \mathcal{B}\left(\omega, D_{x}\right) v^{\varepsilon} ; H_{0}^{-1 / 2}(\partial \mathbb{D})\right\|^{2}\right. \\
& \left.+\left\|\psi v^{\varepsilon} ; H^{1}(\mathbb{D})\right\|^{2}+(1 / \varepsilon)^{2}\left\|\left(\mathcal{B}\left(\omega, D_{x}\right) \kappa\right) v^{\varepsilon} ; H_{0}^{-1 / 2}(\partial \mathbb{D})\right\|^{2}\right\} .
\end{aligned}
$$

After the change of variables $x \mapsto \xi=x / \varepsilon$, this inequality takes the form

$$
\begin{aligned}
(\gamma /|\tau|)^{2} \| & \nabla_{\xi}\left(\kappa_{\varepsilon} v\right) ; L_{2}(\mathbb{D})\left\|^{2}+(\gamma /|\tau|)^{2}\right\| \kappa_{\varepsilon} v ; L_{2}(\mathbb{D}) \|^{2} \\
\leq & c\left\{\left\|\kappa_{\varepsilon} \mathcal{L}\left(D_{\xi}, \theta\right) v ; L_{2}(\mathbb{D})\right\|^{2}+\left\|\kappa_{\varepsilon} \mathcal{B}\left(\omega, D_{\xi}\right) v ; H_{0}^{-1 / 2}(\partial \mathbb{D})\right\|^{2}\right. \\
& \left.\quad+\varepsilon^{4}\left\|\psi_{\varepsilon} v ; L_{2}(\mathbb{D})\right\|^{2}+\varepsilon^{2}\left\|\nabla_{\xi}\left(\psi_{\varepsilon} v\right) ; L_{2}(\mathbb{D})\right\|^{2}+\left\|\left(\mathcal{B}\left(\omega, D_{\xi}\right) \kappa_{\varepsilon}\right) v ; H_{0}^{-1 / 2}(\partial \mathbb{D})\right\|^{2}\right\},
\end{aligned}
$$

where $\theta=\tau /|\tau|$ and $\omega=\xi /|\xi|$. Furthermore,

$$
\begin{aligned}
& \left\|\kappa_{\varepsilon} \mathcal{B}\left(\omega, D_{\xi}\right) v ; H_{0}^{-1 / 2}(\partial \mathbb{D})\right\|^{2} \\
& \quad \leq\left\|\kappa_{\varepsilon} \mathcal{B}\left(\omega, D_{\xi}\right) v ; H_{1 / 2}^{0}(\partial \mathbb{D})\right\|^{2} \leq(c / \varepsilon)\left\|\kappa_{\varepsilon} \mathcal{B}\left(\omega, D_{\xi}\right) v ; L_{2}(\partial \mathbb{D})\right\|^{2}, \\
& \quad\left\|\left(\mathcal{B}\left(\omega, D_{\xi}\right) \kappa_{\varepsilon}\right) v ; H_{0}^{-1 / 2}(\partial \mathbb{D})\right\|^{2} \\
& \quad \leq\left\|\left(\mathcal{B}\left(\omega, D_{\xi}\right) \kappa_{\varepsilon}\right) v ; H_{1 / 2}^{0}(\partial \mathbb{D})\right\|^{2} \leq c \varepsilon\left\|\psi_{\varepsilon} v ; L_{2}(\partial \mathbb{D})\right\|^{2} .
\end{aligned}
$$


Therefore, we have

$$
\begin{aligned}
&(\gamma /|\tau|)^{2}\left\|\nabla_{\xi}\left(\kappa_{\varepsilon} v\right) ; L_{2}(\mathbb{D})\right\|^{2}+(\gamma /|\tau|)^{2}\left\|\kappa_{\varepsilon} v ; L_{2}(\mathbb{D})\right\|^{2} \\
& \leq c\{\left\|\kappa_{\varepsilon} \mathcal{L}\left(D_{\xi}, \theta\right) v ; L_{2}(\mathbb{D})\right\|^{2}+(1 / \varepsilon)\left\|\kappa_{\varepsilon} \mathcal{B}\left(\omega, D_{\xi}\right) v ; L_{2}(\partial \mathbb{D})\right\|^{2} \\
&\left.+\varepsilon^{4}\left\|\psi_{\varepsilon} v ; L_{2}(\mathbb{D})\right\|^{2}+\varepsilon^{2}\left\|\nabla_{\xi}\left(\psi_{\varepsilon} v\right) ; L_{2}(\mathbb{D})\right\|^{2}+\varepsilon\left\|\psi_{\varepsilon} v ; L_{2}(\partial \mathbb{D})\right\|^{2}\right\} .
\end{aligned}
$$

We multiply this inequality by $\varepsilon^{-2 \beta}$, put $\varepsilon=2^{-j}$, and sum all such inequalities for $j=0,1,2, \ldots$, obtaining

$$
\begin{aligned}
(\gamma /|\tau|)^{2}\left\|\nabla_{\xi}\left(\kappa_{\infty} v\right) ; H_{\beta}^{0}(\mathbb{D})\right\|^{2}+(\gamma /|\tau|)^{2}\left\|\kappa_{\infty} v ; H_{\beta}^{0}(\mathbb{D})\right\|^{2} & \\
\leq c\left\{\| \kappa_{\infty} \mathcal{L}\left(D_{\xi}, \theta\right) v\right. & ; H_{\beta}^{0}(\mathbb{D})\left\|^{2}+\right\| \kappa_{\infty} \mathcal{B}\left(\omega, D_{\xi}\right) v ; H_{\beta+1 / 2}^{0}(\partial \mathbb{D}) \|^{2} \\
& \left.+\left\|\psi_{\infty} v ; H_{\beta-1}^{1}(\mathbb{D})\right\|^{2}+\left\|\psi_{\infty} v ; H_{\beta-1 / 2}^{0}(\partial \mathbb{D})\right\|^{2}\right\},
\end{aligned}
$$

where $\kappa_{\infty}, \psi_{\infty} \in \mathcal{C}^{\infty}(\overline{\mathbb{K}})$ are cut-off functions equal to 0 near the vertex of the cone, equal to 1 in a neighborhood of infinity, and satisfying $\kappa_{\infty} \psi_{\infty}=\kappa_{\infty}$.

An estimate in a neighborhood of the edge. Consider the operator

$$
\mathcal{A}: H_{\beta}^{2}(\mathbb{D}) \mapsto H_{\beta}^{0}(\mathbb{D}) \times H_{\beta}^{1 / 2}(\partial \mathbb{D})
$$

given by the formula $v \mapsto \mathcal{A} v:=\left\{A\left(D_{x}\right) v,\left.\mathcal{B}\left(\omega, D_{x}\right) v\right|_{\partial \mathbb{D}}\right\}$. It is known (see [17]) that if $\beta \leq 1$ and the line $\operatorname{Im} \lambda=\beta+n / 2-2$ is free of the spectrum of the pencil $\mathfrak{A}_{N}$, then $\mathcal{A}$ is a monomorphism, and

$$
\left\|v ; H_{\beta}^{2}(\mathbb{D})\right\|^{2} \leq c\left\{\left\|A\left(D_{x}\right) v ; H_{\beta}^{0}(\mathbb{D})\right\|^{2}+\left\|\mathcal{B}\left(\omega, D_{x}\right) v ; H_{\beta}^{1 / 2}(\partial \mathbb{D})\right\|^{2}\right\} .
$$

We also fix cut-off functions $\chi, \psi \in \mathcal{C}_{c}^{\infty}(\overline{\mathbb{D}})$ that are equal to 1 near the edge, are independent of the coordinate $z$, and satisfy $\chi \psi=\chi$. By (97),

$$
\begin{aligned}
& \left\|\chi v ; H_{\beta}^{2}(\mathbb{D}, 1)\right\|^{2} \\
& \quad \leq c\left\{\left\|\chi \mathcal{L}\left(D_{\xi}, \theta\right) v ; H_{\beta}^{0}(\mathbb{D})\right\|^{2}+\left\|\chi \mathcal{B}\left(\omega, D_{\xi}\right) v ; H_{\beta}^{1 / 2}(\partial \mathbb{D})\right\|^{2}+\left\|\psi v ; H_{\beta}^{1}(\mathbb{D}, 1)\right\|^{2}\right\} .
\end{aligned}
$$

The global estimate. We add inequalities (96) and (98). The cut-off function $\kappa_{\infty}$ on the left-hand side can be replaced by 1 . Thus,

$$
\begin{aligned}
&(\gamma /|\tau|)^{2}\left(\left\|\nabla_{\xi} v ; H_{\beta}^{0}(\mathbb{D})\right\|^{2}+\left\|v ; H_{\beta}^{0}(\mathbb{D})\right\|^{2}\right)+\left\|\chi v ; H_{\beta}^{2}(\mathbb{D}, 1)\right\|^{2} \\
& \leq c\left\{\left\|\mathcal{L}\left(D_{\xi}, \theta\right) v ; H_{\beta}^{0}(\mathbb{D})\right\|^{2}+\left\|\chi \mathcal{B}\left(\omega, D_{\xi}\right) v ; H_{\beta}^{1 / 2}(\partial \mathbb{D})\right\|^{2}\right. \\
&+\left\|\kappa_{\infty} \mathcal{B}\left(\omega, D_{\xi}\right) v ; H_{\beta+1 / 2}^{0}(\partial \mathbb{D})\right\|^{2}+\left\|\psi_{\infty} v ; H_{\beta-1 / 2}^{0}(\partial \mathbb{D})\right\|^{2} \\
&\left.+\left\|\psi_{\infty} v ; H_{\beta-1}^{1}(\mathbb{D})\right\|^{2}+\left\|\psi v ; H_{\beta}^{1}(\mathbb{D}, 1)\right\|^{2}\right\} .
\end{aligned}
$$

Changing the variables $\xi \mapsto x=\xi /|\tau|$ and putting $u(x)=v(|\tau| x)$, we obtain

$$
\begin{aligned}
& \gamma^{2}\left(\left\|\nabla_{x} u ; H_{\beta}^{0}(\mathbb{D})\right\|^{2}+|\tau|^{2}\left\|u ; H_{\beta}^{0}(\mathbb{D})\right\|^{2}\right)+\left\|\chi_{\tau} u ; H_{\beta}^{2}(\mathbb{D},|\tau|)\right\|^{2} \\
& \leq c\left\{\left\|\mathcal{L}\left(D_{x}, \tau\right) u ; H_{\beta}^{0}(\mathbb{D})\right\|^{2}+\left\|\chi_{\tau} \mathcal{B}\left(\omega, D_{x}\right) u ; H_{\beta}^{1 / 2}(\partial \mathbb{D})\right\|^{2}\right. \\
& +|\tau|^{2}\left\|\kappa_{\infty, \tau} \mathcal{B}\left(D_{x}\right) u ; H_{\beta+1 / 2}^{0}(\partial \mathbb{D})\right\|^{2}+|\tau|^{2}\left\|\psi_{\tau} u ; H_{\beta}^{1}(\mathbb{D},|\tau|)\right\|^{2} \\
& \left.+\left\|\psi_{\infty, \tau} u ; H_{\beta-1}^{1}(\mathbb{D})\right\|^{2}+|\tau|^{2}\left\|\psi_{\infty, \tau} u ; H_{\beta-1 / 2}^{0}(\partial \mathbb{D})\right\|^{2}\right\} .
\end{aligned}
$$


Now we estimate the last three terms on the right. Let $W$ denote the right-hand side of (61). Since $\beta \leq 1$, we have

$$
\begin{aligned}
& \left\|\psi_{\infty, \tau} u ; H_{\beta-1}^{1}(\mathbb{D})\right\|^{2} \leq \int_{c /|\tau|<r} r^{2(\beta-1)}\left(|\nabla u|^{2}+|u|^{2} / r^{2}\right) \mathrm{d} x \\
& \leq c|\tau|^{2(1-\beta)}\left\{\left\|\nabla u ; L_{2}(\mathbb{D})\right\|^{2}+|\tau|^{2}\left\|u ; L_{2}(\mathbb{D})\right\|^{2}\right\} \leq c \frac{|\tau|^{2(1-\beta)}}{\gamma^{2}} W .
\end{aligned}
$$

Furthermore,

$$
\begin{aligned}
& |\tau|^{2}\left\|\psi_{\tau} u ; H_{\beta}^{1}(\mathbb{D},|\tau|)\right\|^{2} \leq|\tau|^{2}\left(\int_{r<c /|\tau|} r^{2 \beta}\left(|\nabla u|^{2}+|u|^{2} / r^{2}+|\tau|^{2}|u|^{2}\right) \mathrm{d} x\right) \\
& \leq|\tau|^{2}\left(\int_{r<\varepsilon /|\tau|} r^{2 \beta}\left(|\nabla u|^{2}+|u|^{2} / r^{2}+|\tau|^{2}|u|^{2}\right) \mathrm{d} x\right. \\
& \left.\quad+\int_{\varepsilon /|\tau|<r<c /|\tau|} r^{2 \beta}\left(|\nabla u|^{2}+|u|^{2} / r^{2}+|\tau|^{2}|u|^{2}\right) \mathrm{d} x\right) \\
& \leq c \varepsilon^{2} \int_{r<\varepsilon /|\tau|} r^{2(\beta-1)}\left(|\nabla u|^{2}+|u|^{2} / r^{2}+|\tau|^{2}|u|^{2}\right) \mathrm{d} x+c|\tau|^{2(1-\beta)} \int_{\mathbb{D}}\left(|\nabla u|^{2}+|\tau|^{2}|u|^{2}\right) \mathrm{d} x \\
& \leq c \varepsilon^{2}\left\|\chi_{\tau} u ; H_{\beta}^{2}(\mathbb{D},|\tau|)\right\|^{2}+c \frac{|\tau|^{2(1-\beta)}}{\gamma^{2}} W .
\end{aligned}
$$

Finally, for the last term on the right in (99) we obtain

$$
\begin{aligned}
& |\tau|^{2}\left\|\psi_{\infty, \tau} u ; H_{\beta-1 / 2}^{0}(\partial \mathbb{D})\right\|^{2}=|\tau|^{2} \int_{\partial \mathbb{D}} r^{2 \beta-1}\left|\psi_{\infty, \tau} u\right|^{2} \mathrm{~d} S \\
& \quad \leq \frac{|\tau|^{4}}{\gamma^{2} \varepsilon} \int_{c /|\tau|<r} r^{2(\beta-1)}|u|^{2} \mathrm{~d} S+\gamma^{2} \varepsilon \int_{\partial \mathbb{D}} r^{2 \beta}\left|\psi_{\infty, \tau} u\right|^{2} \mathrm{~d} S \\
& \quad \leq C\left(\frac{|\tau|^{2(3-\beta)}}{\gamma^{2} \varepsilon}\left\|u ; L_{2}(\partial \mathbb{D})\right\|^{2}+\gamma^{2} \varepsilon\left\|r^{\beta} \psi_{\infty, \tau} u ; L_{2}(\partial \mathbb{D})\right\|^{2}\right) \\
& \quad \leq C\left(\frac{|\tau|^{2(3-\beta)}}{\gamma^{2} \varepsilon}\left\|u ; H^{1}(\mathbb{D})\right\|^{2}+\gamma^{2} \varepsilon\left\|r^{\beta} \psi_{\infty, \tau} u ; H^{1}(\mathbb{D})\right\|^{2}\right) \\
& \quad \leq C\left(\frac{|\tau|^{2(3-\beta)}}{\gamma^{4} \varepsilon} W+\gamma^{2} \varepsilon\left(\left\|\nabla u ; H_{\beta}^{0}(\mathbb{D})\right\|^{2}+|\tau|^{2}\left\|u ; H_{\beta}^{0}(\mathbb{D})\right\|^{2}\right)\right) .
\end{aligned}
$$

Combining all these estimates, we rewrite (99) in the form

$$
\begin{aligned}
\gamma^{2}\left(\left\|\nabla_{x} u ; H_{\beta}^{0}(\mathbb{D})\right\|^{2}+|\tau|^{2}\left\|u ; H_{\beta}^{0}(\mathbb{D})\right\|^{2}\right)+\left\|\chi_{\tau} u ; H_{\beta}^{2}(\mathbb{D},|\tau|)\right\|^{2} \\
\leq c\left\{\left\|\mathcal{L}\left(D_{x}, \tau\right) u ; H_{\beta}^{0}(\mathbb{D})\right\|^{2}+\left\|\chi_{\tau} \mathcal{B}\left(\omega, D_{x}\right) u ; H_{\beta}^{1 / 2}(\partial \mathbb{D})\right\|^{2}\right. \\
\quad+|\tau|^{2}\left\|\kappa_{\infty, \tau} \mathcal{B}\left(\omega, D_{x}\right) u ; H_{\beta+1 / 2}^{0}(\partial \mathbb{D})\right\|^{2}+\left(\frac{|\tau|^{2(1-\beta)}}{\gamma^{2}}+\frac{|\tau|^{2(3-\beta)}}{\gamma^{4} \varepsilon}\right) W \\
\left.\quad+\varepsilon^{2}\left\|\chi_{\tau} u ; H_{\beta}^{2}(\mathbb{D},|\tau|)\right\|^{2}+\gamma^{2} \varepsilon\left(\left\|\nabla u ; H_{\beta}^{0}(\mathbb{D})\right\|^{2}+|\tau|^{2}\left\|u ; H_{\beta}^{0}(\mathbb{D})\right\|^{2}\right)\right\} .
\end{aligned}
$$

We choose $\varepsilon$ to be sufficiently small and remove the terms with factors $\varepsilon^{2}$ and $\varepsilon$, increasing the constant $c$.

Note that the "constant" $|\tau|^{2(3-\beta)} /\left(\gamma^{4} \varepsilon\right)$ in (95) can be improved in the same way as in 3 . 
5.3. Estimates of solutions with Neumann boundary condition for $\operatorname{dim} \mathbb{K}=2$. We introduce the spaces $H_{\beta}^{2,0}(\mathbb{D})$ and $H_{\beta}^{2,0}(\mathbb{D}, q)$ with the "nonhomogeneous" norms

$$
\begin{aligned}
\left\|w ; H_{\beta}^{2,0}(\mathbb{D})\right\| & =\left(\int_{\mathbb{D}} r^{2(\beta-1)}|w(x)|^{2} \mathrm{~d} x+\sum_{|\alpha|=1}^{2} \int_{\mathbb{D}} r^{2(\beta-2+|\alpha|)}\left|D_{x}^{\alpha} w(x)\right|^{2} \mathrm{~d} x\right)^{1 / 2}, \\
\left\|w ; H_{\beta}^{2,0}(\mathbb{D}, q)\right\| & =\left(\sum_{|\alpha|=1}^{2} \int_{\mathbb{D}} r^{2(\beta-2+|\alpha|)}\left|D_{x}^{\alpha} w(x)\right|^{2} \mathrm{~d} x+q^{2}\left\|w, H_{\beta}^{1}(\mathbb{D}, q)\right\|^{2}\right)^{1 / 2} .
\end{aligned}
$$

For the proof of the following lemma we refer, e.g., to [16].

Lemma 5.3.1. Let $u \in H_{\beta}^{2,0}(\mathbb{K})$, where $\mathbb{K}$ is an angle in the plane and $\beta<1$. Then the limit

$$
u(0)=\lim _{r \rightarrow 0+}|\Omega|^{-1} \int_{\Omega} u(r, \omega) \mathrm{d} \omega
$$

exists, where $\Omega=\mathbb{K} \cap \mathcal{S}^{1}$ and $|\Omega|$ is the length of the arc $\Omega$ in $\mathcal{S}^{1}$. Moreover, $\chi u=$ $\chi(v+u(0)), v \in H_{\beta}^{2}(\mathbb{K})$, and $\chi$ is a cut-off function of class $\mathcal{C}_{c}^{\infty}(\overline{\mathbb{K}})$ equal to 1 near the vertex of $\mathbb{K}$. We have the estimates

$$
\begin{gathered}
\left\|r^{\beta-2}(u-u(0)) ; L_{2}(\mathbb{K} \cap\{|y| \leq 1\})\right\| \leq c\left\|r^{\beta-1} \nabla u ; L_{2}(\mathbb{K} \cap\{|y| \leq 1\})\right\|, \\
|u(0)| \leq c\left\{\left\|r^{\beta-1} \nabla u ; L_{2}(\mathbb{K} \cap\{|y| \leq 1\})\right\|+\left\|u ; L_{2}(\mathbb{K} \cap\{1 / 2 \leq|y| \leq 1\})\right\|\right\} .
\end{gathered}
$$

If $\beta \leq 0$, then $u(0)=0$.

Lemma 5.3.2. Suppose that the conditions of Lemma 5.3.1 are fulfilled and that $\delta \in$ ]0,1[. Then

$$
\begin{array}{r}
|u(0)| \leq c\left\{\delta^{2(1-\beta)}\left\|r^{\beta-1} \nabla u ; L_{2}(\mathbb{K} \cap\{|y| \leq 1\})\right\|\right. \\
\left.+\delta^{-2}\left\|u ; L_{2}(\mathbb{K} \cap\{\delta / 2 \leq|y| \leq 1\})\right\|\right\} .
\end{array}
$$

Proof. It suffices to substitute the function $x \mapsto w(x)=u(\delta x)$ in (101) and change the variables $x \mapsto y=\delta x$.

We introduce the set

$$
\begin{aligned}
& \left\{u \in H_{\beta}^{2,0}(\mathbb{D}, 1) \cap H^{1}(\mathbb{D}):\right. \\
& \left.\quad \mathcal{L}\left(D_{x}, 0\right) u \in L_{2}(\mathbb{D}), \mathcal{B}\left(\omega, D_{x}\right) u \in H_{\beta+1 / 2}^{0}(\partial \mathbb{D}) \cap H^{-1 / 2}(\partial \mathbb{D})\right\} .
\end{aligned}
$$

Proposition 5.3.3. Let $\beta \leq 1$, and let the line $\operatorname{Im} \lambda=\beta-1$ be free of the spectrum of the pencil $\mathfrak{A}_{N}$. Then the functions in the set (103) satisfy the estimate

$$
\begin{aligned}
& \gamma^{2}\left(\left\|\nabla_{x} u ; H_{\beta}^{0}(\mathbb{D})\right\|^{2}+|\tau|^{2}\left\|u ; H_{\beta}^{0}(\mathbb{D})\right\|^{2}\right)+\left\|\chi_{\tau} u ; H_{\beta}^{2,0}(\mathbb{D},|\tau|)\right\|^{2} \\
& \leq c\{\left\|\mathcal{L}\left(D_{x}, \tau\right) u ; H_{\beta}^{0}(\mathbb{D})\right\|^{2}+\left\|\chi_{\tau} \mathcal{B}\left(\omega, D_{x}\right) u ; H_{\beta}^{1 / 2}(\partial \mathbb{D})\right\|^{2} \\
&+|\tau|^{2}\left\|\mathcal{B}\left(\omega, D_{x}\right) u ; H_{\beta+1 / 2}^{0}(\partial \mathbb{D})\right\|^{2} \\
&\left.+\left(|\tau|^{2(3-\beta)} / \gamma^{4}\right)\left(\left\|\mathcal{L}\left(D_{x}, \tau\right) u ; L_{2}(\mathbb{D})\right\|+|\tau|^{2}\left\|\mathcal{B}\left(\omega, D_{x}\right) u ; H^{-1 / 2}(\partial \mathbb{D})\right\|^{2}\right)\right\}
\end{aligned}
$$

where $\sigma \in \mathbb{R}$ and $\gamma \geq \gamma_{0}$; here $\gamma_{0}$ is independent of $\tau=\sigma-i \gamma$.

Proof. An estimate far from the edge. Let $\kappa$ and $\psi$ be the same cut-off functions as at the first step in the proof of Proposition [5.2.1. In inequality (67), we replace the parameter 
$\tau$ with $\tau /|\tau| \varepsilon$ and the function $v$ with $x \mapsto \kappa v^{\varepsilon}=\kappa(x) v(x / \varepsilon)$. This yields

$$
\begin{aligned}
& (\gamma /|\tau| \varepsilon)^{2}\left(\left\|\nabla_{x}\left(\kappa v^{\varepsilon}\right) ; L_{2}(\mathbb{D})\right\|^{2}+(1 / \varepsilon)^{2}\left\|\kappa v^{\varepsilon} ; L_{2}(\mathbb{D})\right\|^{2}\right) \\
& \leq c\left\{\left\|\kappa \mathcal{L}\left(D_{x}, \tau /|\tau| \varepsilon\right) v^{\varepsilon} ; L_{2}(\mathbb{D})\right\|^{2}+(1 / \varepsilon)^{2}\left\|\kappa \mathcal{B}\left(\omega, D_{x}\right) v^{\varepsilon} ; H^{-1 / 2}(\partial \mathbb{D})\right\|^{2}\right. \\
& \left.\quad+\left\|\psi v^{\varepsilon} ; H^{1}(\mathbb{D})\right\|^{2}+(1 / \varepsilon)^{2}\left\|\left(\mathcal{B}\left(\omega, D_{x}\right) \kappa\right) v^{\varepsilon} ; H^{-1 / 2}(\partial \mathbb{D})\right\|^{2}\right\} .
\end{aligned}
$$

After the change of variables $x \mapsto \xi=x / \varepsilon$, this inequality takes the form

$$
\begin{aligned}
(\gamma /|\tau|)^{2}\left(\left\|\nabla_{\xi}\left(\kappa_{\varepsilon} v\right) ; L_{2}(\mathbb{D})\right\|^{2}+\left\|\kappa_{\varepsilon} v ; L_{2}(\mathbb{D})\right\|^{2}\right) & \\
\leq c\left\{\left\|\kappa_{\varepsilon} \mathcal{L}\left(D_{\xi}, \theta\right) v ; L_{2}(\mathbb{D})\right\|^{2}\right. & +(1 / \varepsilon)\left\|\kappa_{\varepsilon} \mathcal{B}\left(\omega, D_{\xi}\right) v ; H^{-1 / 2}(\partial \mathbb{D})\right\|^{2} \\
& +\varepsilon^{4}\left\|\psi_{\varepsilon} v ; L_{2}(\mathbb{D})\right\|^{2}+\varepsilon^{2}\left\|\nabla_{\xi}\left(\psi_{\varepsilon} v\right) ; L_{2}(\mathbb{D})\right\|^{2} \\
& \left.+(1 / \varepsilon)\left\|\left(\mathcal{B}\left(\omega, D_{\xi}\right) \kappa_{\varepsilon}\right) v ; H^{-1 / 2}(\partial \mathbb{D})\right\|^{2}\right\}
\end{aligned}
$$

with $\theta=\tau /|\tau|$. We have

$$
\begin{aligned}
& \left\|\kappa_{\varepsilon} \mathcal{B}\left(\omega, D_{\xi}\right) v ; H^{-1 / 2}(\partial \mathbb{D})\right\|^{2} \\
& \quad \leq\left\|\kappa_{\varepsilon} \mathcal{B}\left(\omega, D_{\xi}\right) v ; L_{2}(\partial \mathbb{D})\right\|^{2},\left\|\left(\mathcal{B}\left(\omega, D_{\xi}\right) \kappa_{\varepsilon}\right) v ; H^{-1 / 2}(\partial \mathbb{D})\right\|^{2} \\
& \quad \leq\left\|\left(\mathcal{B}\left(\omega, D_{\xi}\right) \kappa_{\varepsilon}\right) v ; L_{2}(\partial \mathbb{D})\right\|^{2} \leq c \varepsilon^{2}\left\|\psi_{\varepsilon} v ; L_{2}(\partial \mathbb{D})\right\|^{2} .
\end{aligned}
$$

Multiplying this inequality by $\varepsilon^{-2 \beta}$, putting $\varepsilon=2^{-j}$, and summing all such inequalities for $j=0,1,2, \ldots$, we obtain

$$
\begin{aligned}
(\gamma /|\tau|)^{2}\left(\| \nabla_{\xi}\left(\kappa_{\infty} v\right) ;\right. & \left.H_{\beta}^{0}(\mathbb{D})\left\|^{2}+\right\| \kappa_{\infty} v ; H_{\beta}^{0}(\mathbb{D}) \|^{2}\right) \\
\leq c\left\{\| \kappa_{\infty} \mathcal{L}\left(D_{\xi}, \theta\right) v ;\right. & H_{\beta}^{0}(\mathbb{D})\left\|^{2}+\right\| \kappa_{\infty} \mathcal{B}\left(\omega, D_{\xi}\right) v ; H_{\beta+1 / 2}^{0}(\partial \mathbb{D}) \|^{2} \\
& \left.+\left\|\psi_{\infty} v ; H_{\beta-1}^{1}(\mathbb{D})\right\|^{2}+\left\|\psi_{\infty} v ; H_{\beta-1 / 2}^{0}(\partial \mathbb{D})\right\|^{2}\right\}
\end{aligned}
$$

where $\kappa_{\infty}$ and $\psi_{\infty}$ are cut-off functions of class $\mathcal{C}^{\infty}(\overline{\mathbb{K}})$ equal to 0 near the vertex of $\mathbb{K}$ and to 1 in a neighborhood of infinity and satisfying $\kappa_{\infty} \psi_{\infty}=\kappa_{\infty}$; these cut-off functions are extended to $\mathbb{D}$ and do not depend on the coordinate $z$ of $x=(y, z)$.

An estimate in a neighborhood of the edge.

Lemma 5.3.4. Let $\beta \leq 1$, and let the line $\operatorname{Im} \lambda=\beta-1$ be free of the spectrum of the pencil $\mathfrak{A}_{N}$. Then for any function in the set (103) we have

$$
\begin{aligned}
& \left\|\chi v ; H_{\beta}^{2,0}(\mathbb{D}, 1)\right\|^{2} \\
& \quad \leq c\left\{\left\|\chi \mathcal{L}\left(D_{\xi}, \theta\right) v ; H_{\beta}^{0}(\mathbb{D})\right\|^{2}+\left\|\psi v ; H_{\beta}^{1}(\mathbb{D}, 1)\right\|^{2}+\left\|\chi \mathcal{B}\left(\omega, D_{\xi}\right) v ; H_{\beta}^{1 / 2}(\partial \mathbb{D})\right\|^{2}\right\},
\end{aligned}
$$

where $\chi$ and $\psi$ are cut-off functions in $\mathcal{C}_{c}^{\infty}(\overline{\mathbb{K}})$ equal to 1 near the vertex and such that $\chi \psi=\chi$.

Proof. Consider the operator

$$
\mathcal{A}: H_{\beta}^{2}(\mathbb{D}) \mapsto H_{\beta}^{0}(\mathbb{D}) \times H_{\beta}^{1 / 2}(\partial \mathbb{D})
$$

given by the formula $v \mapsto \mathcal{A} v:=\left\{\mathcal{L}\left(D_{x}, 0\right) v, \mathcal{B}\left(\omega, D_{x}\right) v\right\}$. It is known (see [17]) that, under the assumptions of the lemma, $\mathcal{A}$ is a monomorphism, and

$$
\left\|v ; H_{\beta}^{2}(\mathbb{D})\right\|^{2} \leq c\left\{\left\|\mathcal{L}\left(D_{\xi}, 0\right) v ; H_{\beta}^{0}(\mathbb{D})\right\|^{2}+\left\|\mathcal{B}\left(\omega, D_{\xi}\right) v ; H_{\beta}^{1 / 2}(\partial \mathbb{D})\right\|^{2}\right\} .
$$


Therefore,

$$
\begin{aligned}
& \left\|\chi v ; H_{\beta}^{2}(\mathbb{D}, 1)\right\|^{2} \\
& \quad \leq c\left\{\left\|\chi \mathcal{L}\left(D_{\xi}, \theta\right) v ; H_{\beta}^{0}(\mathbb{D})\right\|^{2}+\left\|\chi \mathcal{B}\left(\omega, D_{\xi}\right) v ; H_{\beta}^{1 / 2}(\partial \mathbb{D})\right\|^{2}+\left\|\psi v ; H_{\beta}^{1}(\mathbb{D}, 1)\right\|^{2}\right\} .
\end{aligned}
$$

By the definition of $H_{\beta}^{2,0}(\mathbb{D}, 1)$, this implies (106).

The global estimate. We add (105) and (106). The cut-off function $\kappa_{\infty}$ on the left-hand side can be replaced by 1 , whence

$$
\begin{aligned}
&(\gamma /|\tau|)^{2}\left(\left\|\nabla_{\xi} v ; H_{\beta}^{0}(\mathbb{D})\right\|^{2}+\left\|v ; H_{\beta}^{0}(\mathbb{D})\right\|^{2}\right)+\left\|\chi v ; H_{\beta}^{2,0}(\mathbb{D}, 1)\right\|^{2} \\
& \leq c\left\{\left\|\mathcal{L}\left(D_{\xi}, \theta\right) v ; H_{\beta}^{0}(\mathbb{D})\right\|^{2}+\left\|\chi \mathcal{B}\left(\omega, D_{\xi}\right) v ; H_{\beta}^{1 / 2}(\partial \mathbb{D})\right\|^{2}\right. \\
&+\left\|\kappa_{\infty} \mathcal{B}\left(\omega, D_{\xi}\right) v ; H_{\beta+1 / 2}^{0}(\partial \mathbb{D})\right\|^{2}+\left\|\psi_{\infty} v ; H_{\beta-1 / 2}^{0}(\partial \mathbb{D})\right\|^{2} \\
&\left.+\left\|\psi_{\infty} v ; H_{\beta-1}^{1}(\mathbb{D})\right\|^{2}+\left\|\psi v ; H_{\beta}^{1}(\mathbb{D}, 1)\right\|^{2}\right\} .
\end{aligned}
$$

We change the variables $\xi \mapsto x=\xi /|\tau|$ and put $u(x)=v(|\tau| x)$. Then the inequality takes the form

$$
\begin{aligned}
& \gamma^{2}\left(\left\|\nabla_{x} u ; H_{\beta}^{0}(\mathbb{D})\right\|^{2}+|\tau|^{2}\left\|u ; H_{\beta}^{0}(\mathbb{D})\right\|^{2}\right)+\left\|\chi_{\tau} u ; H_{\beta}^{2,0}(\mathbb{D},|\tau|)\right\|^{2} \\
& \leq c\left\{\left\|\mathcal{L}\left(D_{x}, \tau\right) u ; H_{\beta}^{0}(\mathbb{D})\right\|^{2}+\left\|\chi_{\tau} \mathcal{B}\left(\omega, D_{x}\right) u ; H_{\beta}^{1 / 2}(\partial \mathbb{D})\right\|^{2}\right. \\
& \quad+|\tau|^{2}\left\|\kappa_{\infty, \tau} \mathcal{B}\left(\omega, D_{x}\right) u ; H_{\beta+1 / 2}^{0}(\partial \mathbb{D})\right\|^{2}+|\tau|^{2}\left\|\psi_{\infty, \tau} u ; H_{\beta-1 / 2}^{0}(\partial \mathbb{D})\right\|^{2} \\
& \left.+\left\|\psi_{\infty, \tau} u ; H_{\beta-1}^{1}(\mathbb{D})\right\|^{2}+|\tau|^{2}\left\|\psi_{\tau} u ; H_{\beta}^{1}(\mathbb{D},|\tau|)\right\|^{2}\right\} .
\end{aligned}
$$

We estimate the last three terms on the right. The quantities $|\tau|^{2}\left\|\psi_{\infty, \tau} u ; H_{\beta-1 / 2}^{0}(\partial \mathbb{D})\right\|^{2}$ and $\left\|\psi_{\infty, \tau} u ; H_{\beta-1}^{1}(\mathbb{D})\right\|^{2}$ can be handled in the same way as at the final step in the proof of Proposition 5.2.1. We have

$$
\begin{aligned}
& |\tau|^{2}\left\|\psi_{\tau} u ; H_{\beta}^{1}(\mathbb{D},|\tau|)\right\|^{2} \leq|\tau|^{2} \int_{r<c /|\tau|} r^{2 \beta}\left(|\nabla u|^{2}+|u|^{2} / r^{2}+|\tau|^{2}|u|^{2}\right) \mathrm{d} x \\
& \leq|\tau|^{2}\left(\int_{r<\varepsilon /|\tau|} r^{2 \beta}\left(|\nabla u|^{2}+|u|^{2} / r^{2}+|\tau|^{2}|u|^{2}\right) \mathrm{d} x\right. \\
& \left.\quad+\int_{\varepsilon /|\tau|<r<c /|\tau|} r^{2 \beta}\left(|\nabla u|^{2}+|u|^{2} / r^{2}+|\tau|^{2}|u|^{2}\right) \mathrm{d} x\right) .
\end{aligned}
$$

Next,

$$
\begin{aligned}
& |\tau|^{2}\left\|\psi_{\tau} u ; H_{\beta}^{1}(\mathbb{D},|\tau|)\right\|^{2} \\
& \quad \leq c|\tau|^{2} \int_{r<\varepsilon /|\tau|} r^{2 \beta}\left(|\nabla u|^{2}+|u|^{2} / r^{2}+|\tau|^{2}|u|^{2}\right) \mathrm{d} x+c|\tau|^{2(1-\beta)} \int_{\mathbb{D}}\left(|\nabla u|^{2}+|\tau|^{2}|u|^{2}\right) \mathrm{d} x .
\end{aligned}
$$

The latter integral is majorized with the help of (67). Moreover,

$$
\begin{aligned}
& c|\tau|^{2} \int_{r<\varepsilon /|\tau|} r^{2 \beta}\left(|\nabla u|^{2}+|u|^{2} / r^{2}+|\tau|^{2}|u|^{2}\right) \mathrm{d} x \\
& \quad \leq c \varepsilon^{2} \int_{r<\varepsilon /|\tau|} r^{2(\beta-1)}\left(|\nabla u|^{2}+|\tau|^{2}|u|^{2}\right) \mathrm{d} x+c|\tau|^{2} \int_{r<\varepsilon /|\tau|} r^{2(\beta-1)}|u|^{2} \mathrm{~d} x .
\end{aligned}
$$


Applying Lemmas 5.3.1 and 5.3.2, we see that, for $\beta>0$,

(109)

$$
\begin{aligned}
|\tau|^{2} \int_{r<\varepsilon /|\tau|} r^{2(\beta-1)}|u(x)|^{2} \mathrm{~d} x \\
\leq \varepsilon^{2} \int_{\mathbb{R}^{d}} \mathrm{~d} z \int_{\mathbb{K} \cap\{r \leq \varepsilon /|\tau|\}} r^{2(\beta-2)}|u(y, z)-u(0, z)|^{2} \mathrm{~d} y \\
\quad+|\tau|^{2} \int_{\mathbb{R}^{d}} \mathrm{~d} z \int_{\mathbb{K} \cap\{r \leq \varepsilon /|\tau|\}} r^{2(\beta-1)}|u(0, z)|^{2} \mathrm{~d} y \\
\leq \varepsilon^{2} \int_{\mathbb{D} \cap\{r \leq 1\}} r^{2(\beta-1)}\left|\nabla_{x} u(x)\right|^{2} \mathrm{~d} x \\
\quad+|\tau|^{2(1-\beta)} \varepsilon^{2 \beta}\left(\delta^{2(1-\beta)} \int_{\mathbb{D} \cap\{r \leq 1\}} r^{2(\beta-1)}\left|\nabla_{x} u(x)\right|^{2} \mathrm{~d} x+\delta^{-2} \int_{\mathbb{D}}|u(x)|^{2} \mathrm{~d} x\right) .
\end{aligned}
$$

Put $\delta=1 /|\tau|$. Choosing $\gamma_{0}$ appropriately, we may assume that $|\tau|>1$. Then estimate (109) implies

$$
\begin{aligned}
& |\tau|^{2} \int_{r<\varepsilon /|\tau|} r^{2(\beta-1)}|u(x)|^{2} \mathrm{~d} x \\
& \quad \leq\left(\varepsilon^{2}+\varepsilon^{2 \beta}\right) \int_{r \leq 1} r^{2(\beta-1)}\left|\nabla_{x} u(x)\right|^{2} \mathrm{~d} x+|\tau|^{2(1-\beta)} \int_{\mathbb{D}}|\tau|^{2}|u(x)|^{2} \mathrm{~d} x .
\end{aligned}
$$

If $\beta \leq 0$, then $u(0, z)=0$ by Lemma 5.3.1. In that case, estimate (109) simplifies:

$$
|\tau|^{2} \int_{r<\varepsilon /|\tau|} r^{2(\beta-1)}|u(x)|^{2} \mathrm{~d} x \leq \varepsilon^{2} \int_{r \leq 1} r^{2(\beta-1)}\left|\nabla_{x} u(x)\right|^{2} \mathrm{~d} x .
$$

Using (108), we see that if $\beta>0$, then

$$
\begin{aligned}
& |\tau|^{2} \int_{r<\varepsilon /|\tau|} r^{2 \beta}\left(|\nabla u|^{2}+|u|^{2} / r^{2}+|\tau|^{2}|u|^{2}\right) \mathrm{d} x \\
& \leq c\left(\varepsilon^{2}+\varepsilon^{2 \beta}\right) \int_{r<\varepsilon /|\tau|} r^{2(\beta-1)}\left(|\nabla u|^{2}+|\tau|^{2}|u|^{2}\right) \mathrm{d} x \\
& \quad+\left(\varepsilon^{2}+\varepsilon^{2 \beta}\right) \int_{\varepsilon /|\tau| \leq r \leq 1} r^{2(\beta-1)}\left|\nabla_{x} u(x)\right|^{2} \mathrm{~d} x+|\tau|^{2(1-\beta)} \int_{\mathbb{D}}|\tau|^{2}|u(x)|^{2} \mathrm{~d} x \\
& \quad \leq c\left(\varepsilon^{2}+\varepsilon^{2 \beta}\right)\left\|\chi_{\tau} u ; H_{\beta}^{2,0}(\mathbb{D},|\tau|)\right\|^{2}+c|\tau|^{2(1-\beta)} \int_{\mathbb{D}}\left(|\tau|^{2}|u(x)|^{2}+\left|\nabla_{x} u(x)\right|^{2}\right) \mathrm{d} x .
\end{aligned}
$$

For $\beta \leq 0$, the same estimate is valid with $\left(\varepsilon^{2}+\varepsilon^{2 \beta}\right)$ replaced by $\varepsilon^{2}$.

Combining the estimates given after (107), we arrive at the inequality

$$
\begin{aligned}
& \gamma^{2}\left(\left\|\nabla_{x} u ; H_{\beta}^{0}(\mathbb{D})\right\|^{2}+|\tau|^{2}\left\|u ; H_{\beta}^{0}(\mathbb{D})\right\|^{2}\right)+\left\|\chi_{\tau} u ; H_{\beta}^{2,0}(\mathbb{D},|\tau|)\right\|^{2} \\
& \leq c\{\left\|\mathcal{L}\left(D_{x}, \tau\right) u ; H_{\beta}^{0}(\mathbb{D})\right\|^{2}+\left\|\chi_{\tau} \mathcal{B}\left(\omega, D_{x}\right) u ; H_{\beta}^{1 / 2}(\partial \mathbb{D})\right\|^{2} \\
&+|\tau|^{2}\left\|\kappa_{\infty, \tau} \mathcal{B}\left(\omega, D_{x}\right) u ; H_{\beta+1 / 2}^{0}(\partial \mathbb{D})\right\|^{2}+|\tau|^{2}\left\|\psi_{\infty, \tau} u ; H_{\beta-1 / 2}^{0}(\partial \mathbb{D})\right\|^{2} \\
&+\left\|\psi_{\infty, \tau} u ; H_{\beta-1}^{1}(\mathbb{D})\right\|^{2}+|\tau|^{2}\left\|\psi_{\tau} u ; H_{\beta}^{1}(\mathbb{D},|\tau|)\right\|^{2}+\frac{|\tau|^{2(3-\beta)}}{\gamma^{4}} W \\
&\left.+\left(\varepsilon^{2}+\varepsilon^{2|\beta|}\right)\left\|\chi_{\tau} u ; H_{\beta}^{2,0}(\mathbb{D},|\tau|)\right\|^{2}+\gamma^{2} \varepsilon\left(\left\|\nabla u ; H_{\beta}^{0}(\mathbb{D})\right\|^{2}+|\tau|^{2}\left\|u ; H_{\beta}^{0}(\mathbb{D})\right\|^{2}\right)\right\} .
\end{aligned}
$$

Choosing $\varepsilon$ sufficiently small and increasing the constant $c$, we can remove all the terms with factor $\varepsilon$ from the right-hand side. 


\section{§6. Boundary VAlue PROBlem IN A CONE IN A SCALE OF WEIGHTED SPACES}

Upon the Fourier transformation $\mathcal{F}_{z \rightarrow \xi}$ along the edge of the wedge $\mathbb{D}=\mathbb{K} \times \mathbb{R}^{m-n}$, the boundary value problems in $\mathbb{D}$ become problems in $\mathbb{K}$ depending on the parameter $\xi \in \mathbb{R}^{m-n}$ (in addition to the parameter $\tau=\sigma-i \gamma$ ). The estimates obtained in the wedge lead to inequalities for the solutions of the problems in $\mathbb{K}$. This enables us to investigate such problems in a scale of weighted spaces and, in particular, to describe the asymptotics of solutions near the vertex. The inverse Fourier transformations $\mathcal{F}_{\xi \rightarrow z}^{-1} \mathcal{F}_{\tau \rightarrow t}^{-1}$ paraphrase the results for the time-dependent problems in the wedge and provide the asymptotics of solutions in a neighborhood of the edge.

First, we present some information on the asymptotics of solutions of elliptic problems in a neighborhood of a conical point. A detailed exposition of this material can be found, e.g., in [17] or 31. Then we turn to problems with parameter $(\xi, \tau)$, using the same method as in [1]-3]. In contrast to [1] and [2], we can do without any additional smoothness of the right-hand side along the edge.

The argument is essentially the same for all problems in a cone that correspond to the problems in a wedge discussed before. For this reason, we basically restrict ourselves to the Dirichlet problem with homogeneous energy estimate.

6.1. On the asymptotics of solutions of elliptic problems in a cone. Let $\mathfrak{A}_{N}$ be the operator pencil of the Neumann problem defined in $\Omega=\mathbb{K} \cap S^{n-1}$,

$$
\mathbb{C} \ni \lambda \mapsto \mathfrak{A}_{N}(\lambda)=\left\{r^{2-i \lambda} A\left(D_{y}, 0\right) r^{i \lambda}, r^{1-i \lambda} \mathcal{B}\left(\omega, D_{y}, 0\right) r^{i l}\right\}
$$

where $r=|y|$ and $\omega=y /|y|$; we write this pencil also in the form $\mathfrak{A}_{N}(\lambda)=\{A(\lambda), \mathcal{B}(\lambda)\}$ (see the notation at the beginning of Subsection 5.1). A function of the form

$$
u(y)=r^{i \lambda} \sum_{q=0}^{k} \frac{1}{q !}(i \ln r)^{q} \varphi^{(k-q)}(\omega)
$$

solves the boundary value problem

$$
\begin{cases}A\left(D_{y}, 0\right) u(y)=0, & y \in \mathbb{K}, \\ \mathcal{B}\left(\omega, D_{y}, 0\right) u(y)=0, & y \in \partial \mathbb{K},\end{cases}
$$

if and only if $\lambda$ is an eigenvalue of the pencil $\mathfrak{A}_{N}$ and $\left\{\varphi^{(0)}, \ldots, \varphi^{(k-q)}\right\}$ is a Jordan chain corresponding to this eigenvalue $\left(\varphi^{(0)}\right.$ is an eigenvector and $\varphi^{(1)}, \ldots, \varphi^{(k-q)}$ are generalized eigenvectors). A solution of the form (110) is called a power-like solution. Let $\kappa_{1} \geq \kappa_{2} \geq \cdots \geq \kappa_{J}$ be the partial multiplicities of an eigenvalue $\lambda_{0}$ and let $\left\{\varphi^{(0, j)}, \ldots, \varphi^{\left(\kappa_{j}-1, j\right)} ; j=1, \ldots, J\right\}$ be a canonical system of Jordan chains. The functions

$$
u^{(k, j)}(y)=r^{i \lambda_{0}} \sum_{q=0}^{k} \frac{1}{q !}(i \ln r)^{q} \varphi^{(k-q, j)}(\omega),
$$

where $k=0, \ldots, \kappa_{j}-1$ and $j=1, \ldots, J$, constitute a basis in the space of power-like solutions corresponding to $\lambda_{0}$. The Green formula

$$
(A(\lambda) \varphi, \psi)_{\Omega}+(\mathcal{B}(\lambda) \varphi, \psi)_{\partial \Omega}=(\varphi, A(\bar{\lambda}+i(n-2)) \psi)_{\Omega}+(\varphi, \mathcal{B}(\bar{\lambda}+i(n-2)) \psi)_{\partial \Omega}
$$

is valid for $\phi$ and $\psi$ in $H^{2}(\Omega)$; thus, the pencil $\mathfrak{A}_{N}(\lambda)^{*}=\mathfrak{A}_{N}(\bar{\lambda}+i(n-2))$ is adjoint to $\mathfrak{A}_{N}(\lambda)$ with respect to the Green formula (113). We also introduce the pencil

$$
\mathbb{C} \ni \lambda \mapsto \mathfrak{A}_{N}^{*}(\lambda):=\left[\mathfrak{A}_{N}(\bar{\lambda})\right]^{*}=\mathfrak{A}_{N}(\lambda+i(n-2)) .
$$

If $\lambda_{0}$ is an eigenvalue of the pencil $\mathfrak{A}_{N}$, then $\bar{\lambda}_{0}$ turns out to be an eigenvalue of $\mathfrak{A}_{N}^{*}$, and the geometric and algebraic multiplicities of $\lambda_{0}$ and $\bar{\lambda}_{0}$ coincide. Canonical systems of 
Jordan chains $\left\{\varphi^{(0, j)}, \ldots, \varphi^{\left(\kappa_{j}-1, j\right)} ; j=1, \ldots, J\right\}$ and $\left\{\psi^{(0, j)}, \ldots, \psi^{\left(\kappa_{j}-1, j\right)} ; j=1, \ldots, J\right\}$ corresponding to $\lambda_{0}$ and $\bar{\lambda}_{0}$ can be chosen as to satisfy the orthogonality and normalization conditions

$$
\begin{aligned}
\sum_{p=0}^{\nu} & \sum_{q=0}^{k} \frac{1}{(\nu+k+1-p-q) !} \\
& \times\left\{\left(\partial_{\lambda}^{\nu+k+1-p-q} A\left(\lambda_{0}\right) \varphi^{(q, \sigma)}, \psi^{(p, \zeta)}\right)_{\Omega}+\left(\partial_{\lambda}^{\nu+k+1-p-q} \mathcal{B}\left(\lambda_{0}\right) \varphi^{(q, \sigma)}, \psi^{(p, \zeta)}\right)_{\partial \Omega}\right\} \\
& =\delta_{\sigma, \zeta} \cdot \delta_{\kappa_{\sigma}-k-1, \nu},
\end{aligned}
$$

where $\delta_{p, q}$ is the Kronecker symbol, $\sigma, \zeta=1, \ldots, J, \nu=0, \ldots, \kappa_{\zeta}-1$, and $k=0, \ldots$, $\kappa_{\sigma}-1$. The functions

$$
v^{(k, j)}(y)=r^{i \bar{\lambda}_{0}-(n-2)} \sum_{q=0}^{k} \frac{1}{q !}(i \ln r)^{q} \psi^{(k-q, j)}(\omega)
$$

form a basis in the space of power-like solutions of problem (111) that correspond to the eigenvalue $\bar{\lambda}_{0}+i(n-2)$. If (114) is fulfilled, we say that the bases (112) and (115) match.

Consider the homogeneous problem

$$
\left\{\begin{array}{l}
\mathcal{L}\left(D_{y}, \xi, \tau\right) u(y)=0, \quad y \in \mathbb{K} \\
\mathcal{B}\left(\omega, D_{y}, \xi\right) u(y)=0, \quad y \in \partial \mathbb{K}
\end{array}\right.
$$

with $\xi \in \mathbb{R}^{m-n}$ and $\tau=\sigma-i \gamma$. Denote by $\left\{u_{\mu}^{(k, j)}\right\}$ and $\left\{v_{\mu}^{(k, j)}\right\}$ the matched bases of power-like solutions of problem (111) corresponding to $\lambda_{\mu}$ and $\bar{\lambda}_{\mu}+i(n-2)$. Substituting $u_{\mu}^{(k, j)}$ in (116) and eliminating the discrepancies successively, we construct the formal series

$$
U_{\mu}^{(k, j)}(y, \xi, \tau)=\sum_{q=0}^{+\infty} r^{i \lambda_{\mu}+q} P_{q}^{(k, j)}(\omega, \xi, \tau, \ln r)
$$

satisfying (116); here the $P_{q}^{(k, j)}$ are polynomials in $\ln r, \xi$, and $\tau$ with coefficients smoothly depending on $\omega \in \bar{\Omega}$. Replacing $\left\{u_{\mu}^{(k, j)}\right\}$ with $\left\{v_{\mu}^{(k, j)}\right\}$, we obtain the formal series

$$
V_{\mu}^{(k, j)}(y, \xi, \tau)=\sum_{q=0}^{+\infty} r^{i\left(\bar{\lambda}_{\mu}+i(n-d-2)\right)+q} Q_{q}^{(k, j)}(\omega, \xi, \tau, \ln r)
$$

satisfying (116); the properties of $Q_{q}^{(k, j)}$ are the same as those of $P_{q}^{(k, j)}$.

The facts listed above are also true for the pencil $\mathfrak{A}_{D}$ of the Dirichlet problem.

If $n>2$, then the line $\operatorname{Im} \lambda=n / 2-1$ is free of the spectra of the pencils $\mathfrak{A}_{N}$ and $\mathfrak{A}_{D}$. For $n=2$, the line $\operatorname{Im} \lambda=n / 2-1=0$ contains one eigenvalue $\lambda_{0}=0$ of the pencil $\mathfrak{A}_{N}$. Its multiplicity is equal to $2 n$. A canonical system of Jordan chains corresponding to $\lambda_{0}$ has the form $\left\{\varphi_{0}^{(0, j)}, \varphi_{0}^{(1, j)}\right\}_{j=1}^{n}$, where $\varphi_{0}^{(0, j)}$ is an eigenvector and $\varphi_{0}^{(1, j)}$ is a generalized eigenvector.

Here we can take $u_{0}^{(0, j)}=\varphi_{0}^{(0, j)}$ and $v_{0}^{(1, j)}=\varphi_{0}^{(1, j)}+\varphi_{0}^{(0, j)} \ln r$ (or, vice versa, $u_{0}^{(1, j)}=$ $\varphi_{0}^{(1, j)}+\varphi_{0}^{(0, j)} \ln r$ and $\left.v_{0}^{(0, j)}=\varphi_{0}^{(0, j)}\right)$ as matched bases of power-like solutions. We construct the formal series $U_{0}^{(k, j)}$ and $V_{0}^{(k, j)}$ for these bases, $k=0,1$.

Assume that

$$
\begin{cases}\mathcal{L}\left(D_{y}, \xi, \tau\right) u(y)=f(y), & y \in \mathbb{K} \\ \mathcal{B}\left(\omega, D_{y}, \xi\right) u(y)=g(y), & y \in \partial \mathbb{K}\end{cases}
$$


where $\chi u \in H_{\beta}^{2}(\mathbb{K}), f \in H_{\beta}^{0}(\mathbb{K}) \cap H_{\gamma}^{0}(\mathbb{K})$, and $g \in H_{\beta}^{1 / 2}(\partial \mathbb{K}) \cap H_{\gamma}^{1 / 2}(\partial \mathbb{K})$ for $\gamma<\beta$; here $\chi$ is a cut-off function in $\mathcal{C}_{c}^{\infty}(\overline{\mathbb{K}})$ equal to 1 near the vertex. If the line $\{\lambda \in \mathbb{C}: \operatorname{Im} \lambda=$ $\gamma+n / 2-2\}$ is free of the spectrum of the pencil $\mathfrak{A}_{N}$, then we have

$$
u=\chi \sum c_{\mu}^{(k, j)} U_{\mu, T}^{(k, j)}+h,
$$

where the remainder $h$ is such that $\chi h \in H_{\gamma}^{2}(\mathbb{K})$. Here $U_{\mu, T}^{(k, j)}$ is the partial sum of the series $U_{\mu}^{(k, j)}$ containing $T$ terms with $T$ such that $\chi r^{i \lambda_{\mu}+(T+1)} P_{T+1}^{(k, j)} \in H_{\gamma}^{2}(\mathbb{K})$. The asymptotic formula for $u$ involves the summands corresponding to the eigenvalues of the pencil in the strip $\{\lambda \in \mathbb{C}: \operatorname{Im} \lambda \in] \gamma+n / 2-2, \beta+n / 2-2[\}$, so $\chi U_{\mu, T}^{(k, j)} \in H_{\beta}^{2}(\mathbb{K})$ and $\chi U_{\mu, T}^{(k, j)} \notin H_{\gamma}^{2}(\mathbb{K})$.

6.2. Strong solutions. We consider the problem

$$
\begin{aligned}
\mathcal{L}\left(D_{y}, \xi, \tau\right) v(y) & =f(y), & & y \in \mathbb{K}, \\
v(y) & =0, & & y \in \partial \mathbb{K} .
\end{aligned}
$$

Let $v$ be in the set

$$
\left\{w \in C^{\infty}(\overline{\mathbb{K}} \backslash 0) \cap H^{1}(\mathbb{K}):\left.w\right|_{\partial \mathbb{K}}=0, \mathcal{L}\left(D_{y}, 0,0\right) w \in L_{2}(\mathbb{K})\right\},
$$

and let $\chi$ belong to the Schwartz space $\mathcal{S}\left(\mathbb{R}^{m-n}\right)$. Consider inequality (29) with the function $\mathbb{D} \ni x=(y, z) \mapsto v(y) \chi(z)$ in the role of $v$. Using the Parseval identity for the Fourier transformation $\mathcal{F}_{z \rightarrow \xi}$ and the fact that $\chi$ is arbitrary, we arrive at the estimate

$$
\gamma^{2} \int_{\mathbb{K}}\left(p^{2}|v(y)|^{2}+|\nabla v(y)|^{2}\right) \mathrm{d} y \leq \int_{\mathbb{K}}\left|\mathcal{L}\left(D_{y}, \xi, \tau\right) v(y)\right|^{2} \mathrm{~d} y,
$$

where $p=\left(|\xi|^{2}+|\tau|^{2}\right)^{1 / 2}, \tau=\sigma-i \gamma, \sigma \in \mathbb{R}$, and $\gamma>0$.

With problem (119), (120) we associate the operator $\mathcal{A}(\xi, \tau)$ in $L_{2}(\mathbb{K})$ given by $\mathcal{A}(\xi, \tau) v=\mathcal{L}\left(D_{y}, \xi, \tau\right) v$ for $v$ in the set (121). In what follows, $\mathcal{A}(\xi, \tau)$ denotes the closure of this operator, with domain $D \mathcal{A}(\xi, \tau)$. Obviously, (122) remains valid for the elements of $D \mathcal{A}(\xi, \tau)$. Thus, the following is true.

Proposition 6.2.1. The kernel of $\mathcal{A}(\xi, \tau)$ is trivial, $\operatorname{Ker} \mathcal{A}(\xi, \tau)=0$, and the range $\mathcal{R} \mathcal{A}(\xi, \tau)$ is closed in $L_{2}(\mathbb{K})$.

Now we prove that $\mathcal{R} \mathcal{A}(\xi, \tau)=L_{2}(\mathbb{K})$. The argument to be employed for this purpose will be used repeatedly for the study of the operator of problem (119), (120) in spaces with weighted norms.

Proposition 6.2.2. $\mathcal{R} \mathcal{A}(\xi, \tau)=L_{2}(\mathbb{K})$.

Proof. It suffices to verify that $\operatorname{Ker} \mathcal{A}(\xi, \tau)^{*}=\{0\}$, where $\mathcal{A}(\xi, \tau)^{*}$ is the operator adjoint to $\mathcal{A}(\xi, \tau)$ with respect to the Green formula. Let $w \in \operatorname{Ker} \mathcal{A}(\xi, \tau)^{*}$. Then the theory of elliptic problems shows that $w \in \mathcal{C}^{\infty}(\overline{\mathbb{K}} \backslash \mathcal{O}) \cap L_{2}(\mathbb{K})$ and

$$
\begin{aligned}
\mathcal{L}\left(D_{y}, \xi, \bar{\tau}\right) w(y) & =0, \quad y \in \mathbb{K}, \\
w(y) & =0, \quad y \in \partial \mathbb{K} .
\end{aligned}
$$

First, we check that $w$ is in $H^{1}(\mathbb{K})$, and consequently, in the set (121). After that it remains to use (122) with $\bar{\tau}$ in place of $\tau$.

Let $\left\{\varkappa_{j}\right\}_{j=-\infty}^{+\infty}$ be a partition of unity on $\overline{\mathbb{K}} \backslash \mathcal{O}$ such that

$$
\operatorname{supp} \varkappa_{j} \subset\left\{y: 2^{j-1}<|y|<2^{j+1}\right\} .
$$

Assume that $\psi_{j} \in C^{\infty}(\overline{\mathbb{K}}), \varkappa_{j} \psi_{j}=\varkappa_{j}$, and

$$
\operatorname{supp} \varkappa_{j} \subset\left\{y: 2^{j-2}<|y|<2^{j+2}\right\} \text {. }
$$


Moreover, we suppose that

$$
\left|D^{\alpha} \varkappa_{j}\right|+\left|D^{\alpha} \psi_{j}\right| \leq c_{\alpha} 2^{-j|\alpha|}
$$

for all multi-indices $\alpha$. For the proof of the following lemma we refer to [15, §4] or [17, Chapter 6].

Lemma 6.2.3. Let $\beta \in \mathbb{R}$, and let $w$ be an arbitrary function of class $H_{\text {loc }}^{2}(\overline{\mathbb{K}} \backslash \mathcal{O})$ satisfying $\left.w\right|_{\partial \mathbb{K} \backslash \mathcal{O}}=0$. If $\xi \neq 0$, then

$$
\left\|\varkappa_{k} w ; H_{\beta}^{2}(\mathbb{K}, 1)\right\|^{2} \leq c\left\{\left\|\psi_{k} A\left(D_{y}, \xi\right) w ; H_{\beta}^{0}(\mathbb{K})\right\|^{2}+2^{2 k(\beta-2)}\left\|\psi_{k} w ; L_{2}(\mathbb{K})\right\|^{2}\right\}
$$

for all $k \in \mathbb{Z}$. If $\xi=0$, then the space $H_{\beta}^{2}(\mathbb{K}, 1)$ on the left must be replaced with $H_{\beta}^{2}(\mathbb{K})$.

We return to the function $w \in \operatorname{Ker} \mathcal{A}(\xi, \tau)^{*}$ and study its behavior at infinity. Since $w \in \operatorname{Ker} \mathcal{A}(\xi, \tau)^{*}$, we have $w \in H_{\text {loc }}^{2}(\overline{\mathbb{K}} \backslash \mathcal{O}) \cap L_{2}(\mathbb{K})$. Then, by (123), $A\left(D_{y}, \xi\right) w \in L_{2}(\mathbb{K})$. We take $\beta \leq 0$ and add inequalities (125) for $k=1,2, \ldots$, obtaining

$$
\left\|\varkappa_{\infty} w ; H_{\beta}^{2}(\mathbb{K}, 1)\right\|^{2} \leq c\left\{\left\|\psi_{\infty} A\left(D_{y}, \xi\right) w ; H_{\beta}^{0}(\mathbb{K})\right\|^{2}+\left\|\psi_{\infty} w ; H_{\beta-2}^{0}(\mathbb{K})\right\|^{2}\right\},
$$

where $\varkappa_{\infty}, \psi_{\infty} \in \mathcal{C}^{\infty}(\overline{\mathbb{K}}), \varkappa_{\infty} \psi_{\infty}=\varkappa_{\infty}$, and both functions are equal to 0 near the vertex of $\mathbb{K}$ and vanish in a neighborhood of infinity. Hence, $\varkappa_{\infty} w \in H_{\beta}^{2}(\mathbb{K}, 1) \cap H_{\beta-1}^{1}(\mathbb{K}, 1)$ for $\beta \leq 0$.

We use the inequality

$$
\begin{aligned}
& (\gamma / p)^{2}\left\|\varkappa_{\infty} w ; H_{\beta}^{1}(\mathbb{K}, 1)\right\|^{2} \\
& \quad \leq c\left\{\left\|\varkappa_{\infty} \mathcal{L}\left(D_{\eta}, \xi / p, \bar{\tau} / p\right) w ; H_{\beta}^{0}(\mathbb{K})\right\|^{2}+\left\|\psi_{\infty} w ; H_{\beta-1}^{1}(\mathbb{K}, 1)\right\|^{2}\right\}
\end{aligned}
$$

(cf. (1.26) in [2]), which can be derived in the same (even a simpler) way as (90) and without the admissibility assumption. Making the change of variables $\eta \mapsto y=\eta / p$ in (127), we see that $\varkappa_{\infty} w \in H_{\beta}^{1}(\mathbb{K}, p)$ for $\beta \leq 0$. Iterating inequality (127), we conclude that $\varkappa_{\infty} w \in H_{\beta}^{1}(\mathbb{K}, p)$ for any $\beta \in \mathbb{R}$.

Near the vertex of $\mathbb{K}$ we have the asymptotic representation

$$
w=\chi \sum c_{\nu}^{(k, j)} V_{\nu, T}^{(k, j)}+v,
$$

where $\chi v \in H^{1}(\mathbb{K})$, and $\chi$ stands for a cut-off function equal to 1 near the vertex (see [17]). The sum involves the terms corresponding to the eigenvalues of the pencil $\mathfrak{A}_{D}$ in the strip $\operatorname{Im} \lambda \in] n / 2-2, n / 2-1[$. The coefficients in the asymptotics satisfy

$$
c_{\nu}^{(k, j)}=\left(\mathcal{L}\left(D_{y}, \xi, \tau\right) \chi u_{\nu}^{(k, j)}, w\right)_{\mathbb{K}},
$$

where, as in (117) and (118), $\left\{u_{\nu}^{(k, j)}\right\}$ and $\left\{v_{\nu}^{(k, j)}\right\}$ are matched bases of power-like solutions of problem (111) (see [17] or [14]). The functions $\chi u_{\nu}^{(k, j)}$ are in $\mathcal{D} \mathcal{A}_{\beta}(\xi, \tau)$; therefore, since $w \in \operatorname{Ker} \mathcal{A}_{\beta}(\xi, \tau)^{*}$, we have

$$
c_{\nu}^{(k, j)}=\left(\mathcal{A}(\xi, \tau) \chi u_{\nu}^{(k, j)}, w\right)_{\mathbb{K}}=\left(\chi u_{\nu}^{(k, j)}, \mathcal{A}(\xi, \tau)^{*} w\right)_{\mathbb{K}}=0 .
$$

This implies that $\chi w \in H^{1}(\mathbb{K})$.

Thus, $w$ satisfies (123), (124) and is such that $\chi w \in H^{1}(\mathbb{K})$ and $\varkappa_{\infty} w \in H_{\beta}^{1}(\mathbb{K}, p)$ for any $\beta \in \mathbb{R}$. Therefore, $w$ belongs to the set (121). Using (122) with $\bar{\tau}$ in place of $\tau$, we see that $w \equiv 0$.

Definition 6.2.4. A solution of the equation $\mathcal{A}(\xi, \tau) v=f$ is called a strong solution of problem (119), (120) with $f \in L_{2}(\mathbb{K})$. 
Theorem 6.2.5. For every $f \in L_{2}(\mathbb{K})$ and every $\tau=\sigma-i \gamma(\sigma \in \mathbb{R}$ and $\gamma>0)$, there exists a strong solution $v$ of problem (119), (120). We have

$$
\gamma^{2}\left(p^{2}\left\|v ; L_{2}(\mathbb{K})\right\|^{2}+\left\|\nabla v ; L_{2}(\mathbb{K})\right\|^{2}\right) \leq c\left\|f ; L_{2}(\mathbb{K})\right\|^{2},
$$

where $p=\left(|\xi|^{2}+|\tau|^{2}\right)^{1 / 2}$. The constant $c$ is independent of $(\xi, \tau)$.

Remark 6.2.6. Theorem 6.2.5 remains valid for problem (119), (120) with $\tau$ replaced by $\bar{\tau}$.

6.3. The operator of problem (119), (120) in a scale of weighted spaces. We introduce the set

$$
\left\{u \in H_{\beta}^{2}(\mathbb{K}, p) \cap H^{1}(\mathbb{K}): \mathcal{L}\left(D_{y}, 0,0\right) u \in L_{2}(\mathbb{K}),\left.u\right|_{\partial \mathbb{K}}=0\right\} .
$$

Assume that the line $\operatorname{Im} \lambda=\beta+n / 2-2$ is free of the spectrum of the pencil $\mathfrak{A}_{D}$, and that $\beta \leq 1$. Let $v$ be in the set (128), and let $\psi$ belong to $\mathcal{S}\left(\mathbb{R}^{m-n}\right)$. We substitute the function $\mathbb{D} \ni x=(y, z) \mapsto v(y) \psi(z)$ for $v$ in (88). Using the Parseval identity for the Fourier transformation $\mathcal{F}_{z \rightarrow \xi}$ and the fact that $\psi$ is arbitrary, we arrive at the estimate

$$
\begin{aligned}
& \gamma^{2}\left\|v ; H_{\beta}^{1}(\mathbb{K}, p)\right\|^{2}+\left\|\chi_{\tau} v ; H_{\beta}^{2}(\mathbb{K}, p)\right\|^{2} \\
& \quad \leq c\left\{\left\|\mathcal{L}\left(D_{y}, \xi, \tau\right) v ; H_{\beta}^{0}(\mathbb{K})\right\|^{2}+\left(|\tau|^{2(1-\beta)} / \gamma^{2}\right)\left\|\mathcal{L}\left(D_{y}, \xi, \tau\right) v ; L_{2}(\mathbb{K})\right\|^{2}\right\}
\end{aligned}
$$

where $p=\left(|\xi|^{2}+|\tau|^{2}\right)^{1 / 2}$ and $\tau=\sigma-i \gamma(\sigma \in \mathbb{R}, \gamma>0)$. The constant $c$ is independent of $(\xi, \tau)$. We introduce the spaces $\mathcal{D} H_{\beta}(\mathbb{K}, \xi, \tau)$ and $\mathcal{R} H_{\beta}(\mathbb{K}, \xi, \tau)$ with the norms

$$
\begin{aligned}
\left\|u ; \mathcal{D} H_{\beta}(\mathbb{K}, \xi, \tau)\right\| & =\left(\gamma^{2}\left\|v ; H_{\beta}^{1}(\mathbb{K}, p)\right\|^{2}+\left\|\chi_{\tau} v ; H_{\beta}^{2}(\mathbb{K}, p)\right\|^{2}\right)^{1 / 2} \\
\left\|f ; \mathcal{R} H_{\beta}(\mathbb{K}, \xi, \tau)\right\| & =\left(\left\|f ; H_{\beta}^{0}(\mathbb{K})\right\|^{2}+\left(|\tau|^{2(1-\beta)} / \gamma^{2}\right)\left\|f ; L_{2}(\mathbb{K})\right\|^{2}\right)^{1 / 2} .
\end{aligned}
$$

With problem (119), (120), we associate the operator

$$
\mathcal{A}_{\beta}(\xi, \tau): \mathcal{D} H_{\beta}(\mathbb{K}, \xi, \tau) \longrightarrow \mathcal{R} H_{\beta}(\mathbb{K}, \xi, \tau)
$$

given by $\mathcal{A}_{\beta}(\xi, \tau) v:=\mathcal{L}\left(D_{y}, \xi, \tau\right) v$, the set (128) being its domain. It is not hard to see that this operator admits closure. In what follows, $\mathcal{A}_{\beta}(\xi, \tau)$ and $\mathcal{D} \mathcal{A}_{\beta}(\xi, \tau)$ denote the closed operator and its domain. Estimate (129) leads to the following statement.

Proposition 6.3.1. Suppose that $\beta \leq 1$, the line $\operatorname{Im} \lambda=\beta+n / 2-2$ is free of the spectrum of the pencil $\mathfrak{A}_{D}$, and $\tau=\sigma-i \gamma$, where $\sigma \in \mathbb{R}$ and $\gamma>0$. Then the kernel $\operatorname{Ker} \mathcal{A}_{\beta}(\xi, \tau)$ is trivial and the range $\mathcal{R} \mathcal{A}_{\beta}(\xi, \tau)$ is closed in $\mathcal{R} H_{\beta}(\mathbb{K}, \xi, \tau)$.

When studying the dependence of $\mathcal{R} \mathcal{A}_{\beta}(\xi, \tau)$ on the parameter $\beta$, we shall apply the following proposition describing the special solutions of the homogeneous problem (119), (120) that grow near the vertex of $\mathbb{K}$.

Proposition 6.3.2. Let $\lambda_{\mu}$ be an eigenvalue of $\mathfrak{A}_{D}$ such that $\operatorname{Im} \lambda_{\mu}<n / 2-1$. Let $\left\{v_{\mu}^{(k, j)}\right\}$ be a basis in the space of power-like solutions corresponding to $\bar{\lambda}_{\mu}+i(n-2)$. Then there exist functions $y \mapsto w_{\mu}^{(k, j)}(y, \xi, \tau)$ of class $\mathcal{C}^{\infty}(\overline{\mathbb{K}} \backslash \mathcal{O})$ satisfying the homogeneous problem (119), (120) and such that

$$
w_{\mu}^{(k, j)}(y, \xi, \tau)=\chi V_{\mu, T}^{(k, j)}(y, \xi, \tau)+\rho(y, \xi, \tau),
$$

where $V_{\mu, T}^{(k, j)}$ is a partial sum of the series (118) with sufficiently large $T$. The function $\rho$ depends on $\mu, k, j, T$, and $\chi$, belongs to $\mathcal{C}^{\infty}(\overline{\mathbb{K}} \backslash \mathcal{O})$, and satisfies $\rho(y, \xi, \tau)=O\left(|y|^{h}\right)$ with $h=\min \left\{-\operatorname{Im} \lambda_{\mu}: \operatorname{Im} \lambda_{\mu} \leq n / 2-1\right\}$ as $y \rightarrow 0$ for fixed $(\xi, \tau)$. Moreover, $(1-\chi) \rho \in$ $H_{\beta}^{2}(\mathbb{K}, p)$ for any $\beta \in \mathbb{R}$. The functions $w_{\mu}^{(k, j)}$ are uniquely determined and do not depend on $T$ and $\chi$. 
Proof. 1. We verify the uniqueness of $w_{\mu}^{(k, j)}$. Assume that there exists another function $\widetilde{w}_{\mu}^{(k, j)}$ with representation

$$
\widetilde{w}_{\mu}^{(k, j)}=\widetilde{\chi} V_{\mu, \widetilde{T}}^{(k, j)}+\widetilde{\rho}
$$

and the same properties. Then the difference $w_{\mu}^{(k, j)}-\widetilde{w}_{\mu}^{(k, j)}$ belongs to the set (121) and satisfies the homogeneous problem (119), (120). Therefore, by (122), we have $w_{\mu}^{(k, j)}=$ $\widetilde{w}_{\mu}^{(k, j)}$.

2. Now we prove the existence of $w_{\mu}^{(k, j)}$. Let

$$
f_{T}:=\mathcal{L}\left(D_{y}, \xi, \tau\right) \chi V_{\mu, T}^{(k, j)}=\chi \mathcal{L}\left(D_{y}, \xi, \tau\right) V_{\mu, T}^{(k, j)}+[\mathcal{L}, \chi] V_{\mu, T}^{(k, j)} .
$$

Obviously, the support supp $f_{T} \subset \overline{\mathbb{K}}$ is compact, and for sufficiently large $T$ the function $f_{T}$ decays rapidly as $|y| \rightarrow 0$. Hence, $f_{T} \in L_{2}(\mathbb{K})$ for large $T$. By Theorem 6.2.5 and Remark6.2.6, there exists a function $\rho \in \mathcal{D} \mathcal{A}(\xi, \tau)$ that solves the equation $\mathcal{A}(\xi, \tau) \rho=f_{T}$. We put $w_{\mu}^{(k, j)}=\chi V_{\mu, T}^{(k, j)}-\rho$.

3. We show that these functions $w_{\mu}^{(k, j)}$ possess all the required properties. Since problem (119), (120) is elliptic (for fixed $(\xi, \tau)$ ), and the right-hand side $f_{T}$ belongs to $\mathcal{C}^{\infty}(\overline{\mathbb{K}} \backslash \mathcal{O})$, we have $\rho \in \mathcal{C}^{\infty}(\overline{\mathbb{K}} \backslash \mathcal{O})$. Moreover, since $f_{T}$ decays rapidly near the vertex of $\mathbb{K}$, the function $\rho$ admits asymptotics as $|y| \rightarrow 0$. The fact that $\rho \in \mathcal{D} \mathcal{A}(\xi, \tau)$ allows us to obtain the required asymptotic estimate $\rho=O\left(|y|^{h}\right)$ for small $|y|$. Finally, to see that $(1-\chi) \rho \in H_{\beta}^{2}(\mathbb{K}, p)$ for any $\beta \in \mathbb{R}$, we can use the argument based on Lemma 6.2.3 and employed in the proof of Proposition 6.2.2.

Remark 6.3.3. Proposition 6.3.2 remains valid for problem (119), (120) with $\bar{\tau}$ in place of $\tau$.

We turn to the dependence of the properties of the operator $\mathcal{A}_{\beta}(\xi, \tau)$ on $\beta$. Let $1>$ $\beta_{1}>\beta_{2}>\cdots$ be all numbers in ] $-\infty, 1\left[\right.$ such that every line $\left\{\lambda \in \mathbb{C}: \operatorname{Im} \lambda=\beta_{k}+n / 2-2\right\}$ contains at least one eigenvalue of the pencil $\mathfrak{A}_{D}$. We denote by $S_{m}$ the total multiplicity of the eigenvalues of $\mathfrak{A}_{D}$ in the strip $\left\{\lambda \in \mathbb{C}: \operatorname{Im} \lambda \in\left[\beta_{m}+n / 2-2, \beta_{1}+n / 2-2\right]\right\}$.

Definition 6.3.4. Assume that $f \in \mathcal{R} H_{\beta}(\mathbb{K}, \xi, \tau)$. A solution of the equation

$$
\mathcal{A}_{\beta}(\xi, \tau) v=f
$$

is called a strong $\beta$-solution of problem (119), (120).

The proof of the following theorem can be obtained by the same argument as in 2, 3 ] (in similar situations).

Theorem 6.3.5. A) Suppose that $\left.\beta \in] \beta_{1}, 1\right]$ and $\gamma>0$. Then, for any $f \in \mathcal{R} H_{\beta}(\mathbb{K}, \xi, \tau)$, problem (119), (120) with right-hand side $f$ admits a unique strong $\beta$-solution $v$. The estimate

$$
\left\|v ; \mathcal{D} H_{\beta}(\mathbb{K}, \xi, \tau)\right\| \leq c\left\|f ; \mathcal{R} H_{\beta}(\mathbb{K}, \xi, \tau)\right\|
$$

is true with $c$ independent of $(\xi, \tau)$.

B) Suppose that $\beta \in] \beta_{m+1}, \beta_{m}[$ and $\gamma>0$. Then a strong $\beta$-solution to the problem (119), (120) exists only if the orthogonality conditions

$$
\left(f, w_{\mu}^{(k, j)}(\cdot, \xi, \bar{\tau})\right)_{\mathbb{K}}=0
$$

are fulfilled for all $w_{\mu}^{(k, j)}(\cdot, \xi, \bar{\tau})$ corresponding to the eigenvalues in the strip $\{\lambda \in \mathbb{C}$ : $\left.\operatorname{Im} \lambda \in\left[\beta_{m}+n / 2-2, \beta_{1}+n / 2-2\right]\right\}$. Such a solution satisfies the same estimate as in part A). 
6.4. Asymptotics of solutions of the problem in a cone. By Theorem 6.3.5 a (unique) strong $\beta$-solution in $\mathcal{D} H_{\beta}(\mathbb{K}, \xi, \tau)$ of problem (119), (120) exists if and only if the function $f \in \mathcal{R} H_{\beta}(\mathbb{K}, \xi, \tau)$ satisfies the orthogonality conditions. Since $\mathcal{R} H_{\beta}(\mathbb{K}, \xi, \tau) \subset$ $L_{2}(\mathbb{K})$, Proposition 6.2.2 shows that there is a unique strong solution for any $f \in$ $\mathcal{R} H_{\beta}(\mathbb{K}, \xi, \tau)$. The following Proposition 6.4.1 shows that such a solution admits asymptotics modulo $\mathcal{D} H_{\beta}(\mathbb{K}, \xi, \tau)$. We restrict ourselves to the case where the asymptotics involves only the leading terms of the series $U_{\mu}^{(k, j)}$. The proof is similar to those in 2, 3].

Proposition 6.4.1. Assume that $\gamma>0, \beta \in] \beta_{q+1}, \beta_{q}\left[\right.$, and $0<\beta_{q}-\beta<1$. Suppose also that $f \in \mathcal{R} H_{\beta}(\mathbb{K}, \xi, \tau)$ and that

$$
\left(f, w_{\mu}^{(k, j)}(\cdot, \xi, \bar{\tau})\right)_{\mathbb{K}}=0
$$

for all $w_{\mu}^{(k, j)}(\cdot, \xi, \bar{\tau})$ corresponding to the eigenvalues of $\mathfrak{A}_{D}$ in the strip $\{\lambda \in \mathbb{C}: \operatorname{Im} \lambda \in$ $\left.\left[\beta_{q-1}+n / 2-2, \beta_{1}+n / 2-2\right]\right\}$. Then the strong solution $u$ of problem (119), (120) admits the representation

$$
u(y, \xi, \tau)=\chi(p y) \sum_{\nu} \sum_{k, j} c_{\nu}^{(k, j)}(\xi, \tau) u_{\nu}^{(k, j)}(y)+w(y, \xi, \tau) .
$$

The outer summation is over the eigenvalues $\lambda_{\nu}$ of the pencil located on the line $\operatorname{Im} \lambda=$ $\beta_{q}+n / 2-2$, while the inner summation is over all the elements $\left\{u_{\nu}^{(k, j)}\right\}$ of the basis of power-like solutions corresponding to $\lambda_{\nu}$. The remainder $w$ belongs to $\mathcal{D} H_{\beta}(\mathbb{K}, \xi, \tau)$. The coefficients in the asymptotics are given by

$$
c_{\nu}^{(k, j)}(\xi, \tau)=p^{i \lambda_{\nu}} \sum_{q=0}^{\kappa_{j, \nu}-k-1} \frac{1}{q !}(i \log p)^{q} d_{\nu}^{(k+q, j)}(\xi, \tau)
$$

with

$$
d_{\nu}^{(k, j)}(\xi, \tau)=p^{-2}\left(f(\cdot / p, \xi, \tau), w_{\nu}^{(k, j)}(\cdot, \xi / p, \bar{\tau} / p)\right)_{\mathbb{K}} .
$$

Moreover, we have

$$
\begin{aligned}
\left\|w ; \mathcal{D} H_{\beta}(\mathbb{K}, \xi, \tau)\right\| & \leq c\left\|f ; \mathcal{R} H_{\beta}(\mathbb{K}, \xi, \tau)\right\|, \\
\left|d_{\nu}^{(k, j)}(\xi, \tau)\right| & \leq c p^{\beta+n / 2-2}\left\|f ; \mathcal{R} H_{\beta}(\mathbb{K}, \xi, \tau)\right\|,
\end{aligned}
$$

where the constant $c$ is independent of $\xi, \tau$ and $f, g$.

\section{§7. On The TIME-DEPENDENT PROBLEM IN A WEDGE}

We present some basic results for the nonstationary problem (22), (23) with constant coefficients in the cylinder $Q=\mathbb{D} \times \mathbb{R}$. The results are obtained with the help of the inverse Fourier transformation $\mathcal{F}_{(\xi, \tau) \rightarrow(z, t)}^{-1}$ from those established for the problem (119), (120) in $\mathbb{K}$.

We introduce $H_{\beta}^{s}(Q)$ as the completion of $C_{c}^{\infty}((\overline{\mathbb{D}} \backslash M) \times \mathbb{R})$ in the norm

$$
\left\|w ; H_{\beta}^{s}(Q)\right\|=\left(\sum_{|\alpha| \leq s} \int_{\mathbb{D}} \int_{\mathbb{R}}|y|^{2(\beta-s+|\alpha|)}\left|D_{x, t}^{\alpha} w(x, t)\right|^{2} \mathrm{~d} x \mathrm{~d} t\right)^{1 / 2},
$$

where $x=(y, z) \in \mathbb{D}$. The norm in the space $H_{\beta}^{s}(Q, q)$ with positive parameter $q$ is defined by

$$
\left\|w ; H_{\beta}^{s}(Q, q)\right\|=\left(\sum_{k=0}^{s} q^{2 k}\left\|u ; H_{\beta}^{s-k}(Q)\right\|^{2}\right)^{1 / 2} .
$$


Let $V_{\beta}^{s}(Q, \gamma), \gamma>0$, denote the space with the norm

$$
\left\|w ; V_{\beta}^{s}(Q, \gamma)\right\|=\left\|w^{\gamma} ; H_{\beta}^{s}(Q, \gamma)\right\|,
$$

where $w^{\gamma}(x, t):=\exp (-\gamma t) w(x, t)$.

We consider the problem (22), (23) with constant coefficients and the corresponding problem (119), (120).

Definition 7.1. Assume that $f \in V_{0}^{0}(\mathcal{Q}, \gamma)$ and that $\widehat{u}(y, \xi, \tau)$ is the strong solution of problem (119), (120) in $\mathbb{K}$ with the right-hand side

$$
\widehat{f}(y, \xi, \tau)=\mathcal{F}_{(z, t) \rightarrow(\xi, \tau)} f(y, z, t) .
$$

The function $u$ given by

$$
u(y, z, t)=\mathcal{F}_{(\xi, \tau) \rightarrow(z, t)}^{-1} \widehat{u}(y, \xi, \tau),
$$

is called a strong solution of problem (22), (23).

The next assertion follows from Theorem 6.2.5.

Theorem 7.2. For any $f \in V_{0}^{0}(\mathcal{Q}, \gamma)$ with $\gamma>0$, problem (22), (23) admits a unique strong solution $v$. We have

$$
\gamma\left\|v ; V_{0}^{1}(\mathcal{Q}, \gamma)\right\| \leq c\left\|f ; V_{0}^{0}(\mathcal{Q}, \gamma)\right\|
$$

where $c$ is a constant independent of $\gamma$.

Let $\chi \in \mathcal{C}^{\infty}(\overline{\mathbb{K}})$ be a cut-off function equal to 1 near the conical point $\mathcal{O}$, and let

$$
\begin{aligned}
X u(y, z, t) & =\mathcal{F}_{(\xi, \tau) \rightarrow(z, t)}^{-1} \chi(p y) \mathcal{F}_{\left(z^{\prime}, t^{\prime}\right) \rightarrow(\xi, \tau)} u\left(y, z, t^{\prime}\right), \\
\Lambda^{\mu} u(y, z, t) & =\mathcal{F}_{\tau \rightarrow t}^{-1}|\tau|^{\mu} \mathcal{F}_{t^{\prime} \rightarrow \tau} u\left(y, z, t^{\prime}\right) .
\end{aligned}
$$

We introduce the spaces $\mathcal{D} V_{\beta}(\mathcal{Q}, \gamma), \mathcal{R} V_{\beta}(\mathcal{Q}, \gamma)$ with the norms

$$
\begin{aligned}
\left\|u ; \mathcal{D} V_{\beta}(\mathcal{Q}, \gamma)\right\| & =\left(\gamma^{2}\left\|u ; V_{\beta}^{1}(\mathcal{Q}, \gamma)\right\|^{2}+\left\|X u ; V_{\beta}^{2}(\mathcal{Q}, \gamma)\right\|^{2}\right)^{1 / 2} \\
\left\|f ; \mathcal{R} V_{\beta}(\mathcal{Q}, \gamma)\right\| & =\left(\left\|f ; V_{\beta}^{0}(\mathcal{Q}, \gamma)\right\|^{2}+\left(1 / \gamma^{2}\right)\left\|\Lambda^{1-\beta} f ; V_{0}^{0}(\mathcal{Q}, \gamma)\right\|^{2}\right)^{1 / 2}
\end{aligned}
$$

Definition 7.3. Assume that $f \in \mathcal{R} V_{\beta}(\mathcal{Q}, \gamma)$ and that $\widehat{u}(y, \xi, \tau)$ is the strong $\beta$-solution of problem (119), (120) in $\mathbb{K}$ with the right-hand side $\widehat{f}(y, \xi, \tau)=\mathcal{F}_{(z, t) \rightarrow(\xi, \tau)} f(y, z, t)$. The function $u$ given by $u(y, z, t)=\mathcal{F}_{(\xi, \tau) \rightarrow(z, t)}^{-1} \widehat{u}(y, \xi, \tau)$ is called a strong $\beta$-solution of problem (22), (23) (with constant coefficients).

The following statement is a consequence of Theorem 6.3.5.

Theorem 7.4. A) Assume that $\beta \in] \beta_{1}, 1\left[\right.$ and $\gamma>0$. Then, for $f \in \mathcal{R} V_{\beta}(\mathcal{Q}, \gamma)$, problem (22), (23) with constant coefficients admits a unique strong $\beta$-solution $v$. We have

$$
\left\|v ; \mathcal{D} V_{\beta}(\mathcal{Q}, \gamma)\right\| \leq c\left\|f ; \mathcal{R} V_{\beta}(\mathcal{Q}, \gamma)\right\|
$$

where the constant $c$ is independent of $\gamma$.

B) Suppose that $\beta \in] \beta_{m+1}, \beta_{m}\left[\right.$ and $f \in \mathcal{R} V_{\beta}(\mathcal{Q}, \gamma)$. A strong $\beta$-solution of problem (22), (23) exists and is unique if and only if

$$
\left(\widehat{f}(\cdot, \xi, \tau), w_{\mu}^{(k, j)}(\cdot, \xi, \bar{\tau})\right)_{\mathbb{K}}=0
$$

for all $\xi \in \mathbb{R}$, all $\tau \in \mathbb{R}-i \gamma$, and all the functions $w_{\mu}^{(k, j)}(\cdot, \xi, \bar{\tau})$ corresponding to the eigenvalues of the pencil $\mathfrak{A}_{D}$ in the strip $\left\{\lambda \in \mathbb{C}: \operatorname{Im} \lambda \in\left[\beta_{m}+n / 2-2, \beta_{1}+n / 2-2\right]\right\}$. This solution satisfies the same estimate as in part $\mathrm{A})$. 
Now we present the asymptotics of solutions of the time-dependent problem in a neighborhood of the edge. Theorem 6.4.1 leads to the following result.

Theorem 7.5. Assume that $\left.f \in \mathcal{R} V_{\beta}(\mathcal{Q}, \gamma), \beta \in\right] \beta_{q+1}, \beta_{q}\left[\right.$, and $0<\beta_{q}-\beta<1$. Suppose also that

$$
\left(\widehat{f}(\cdot, \xi, \tau), w_{\mu}^{(k, j)}(\cdot, \xi, \bar{\tau})\right)_{\mathbb{K}}=0
$$

for all $\tau \in \mathbb{R}-i \gamma$, all $\xi \in \mathbb{R}^{m-n}$, and all the functions $w_{\mu}^{(k, j)}(\cdot, \xi, \bar{\tau})$ corresponding to the eigenvalues of the pencil $\mathfrak{A}_{D}$ in the strip $\left\{\lambda \in \mathbb{C}: \operatorname{Im} \lambda \in\left[\beta_{q-1}+n / 2-2, \beta_{1}+n / 2-2\right]\right\}$. Then the strong solution $u$ of problem (22), (23) (with constant coefficients) admits the asymptotic representation

$$
u(y, z, t)=\sum_{\nu} \sum_{k, j}\left(X \widetilde{c}_{\nu}^{(k, j)}\right)(y, z, t) \cdot u_{\nu}^{(k, j)}(y)+w(y, z, t) .
$$

The outer summation is over the eigenvalues $\lambda_{\nu}$ of the pencil on the line $\operatorname{Im} \lambda=\beta_{q}+$ $n / 2-2$. The inner summation is over all the functions $\left\{u_{\nu}^{(k, j)}\right\}$ corresponding to $\lambda_{\nu}$. The remainder $w$ is in $\mathcal{D} V_{\beta}(\mathcal{Q}, \gamma)$. The coefficients in the asymptotics are defined by

$$
\widetilde{c}_{\nu}^{(k, j)}(z, t)=\mathcal{F}_{(\xi, \tau) \rightarrow(z, t)}^{-1} c_{\nu}^{(k, j)}(\xi, \tau),
$$

where

$$
c_{\nu}^{(k, j)}(\xi, \tau)=p^{i \lambda_{\nu}} \sum_{q=0}^{\kappa_{j, \nu}-k-1} \frac{1}{q !}(i \log p)^{q} d_{\nu}^{(k+q, j)}(\xi, \tau)
$$

and

$$
d_{\nu}^{(k, j)}(\xi, \tau)=p^{-2}\left(f(\cdot / p, \xi, \tau), w_{\nu}^{(k, j)}(\cdot, \xi / p, \bar{\tau} / p)\right)_{\mathbb{K}} ;
$$

as before, $p=\left(|\xi|^{2}+|\tau|^{2}\right)^{1 / 2}$. Moreover,

$$
\begin{aligned}
\left\|e^{-\gamma t} \widetilde{d}_{\nu}(\cdot) ; H^{2-n / 2-\beta}\left(\mathbb{R}^{m-n+1}\right)\right\| & \leq c\left\|f ; \mathcal{R} V_{\beta}(\mathcal{Q}, \gamma)\right\|, \\
\left\|w ; \mathcal{D} V_{\beta}(\mathcal{Q}, \gamma)\right\| & \leq\left\|f ; \mathcal{R} V_{\beta}(\mathcal{Q}, \gamma)\right\| .
\end{aligned}
$$

We explain the role of the operator $X$ in the asymptotic formula (132). The representation for $c_{\nu}^{(k, j)}$ and estimate (133) (which is sharp in the sense of smoothness) show that the coefficient $c_{\nu}^{(k, j)}$ can be highly nonsmooth. Therefore, the term $c_{\nu}^{(k, j)}(z, t) u_{\nu}^{(k, j)}(y)$ included in the asymptotics (132) instead of $\left.\left(X c_{\nu}^{(k, j)}\right)(y, z, t) u_{\nu}^{(k, j)}(y)\right)$ could be less smooth than the solution $u$ itself. The operator $X$ determines a smoothing extension of $c_{\nu}^{(k, j)}$ to the interior of the cylinder and enables us to avoid the unnatural situation mentioned above. If the function $c_{\nu}^{(k, j)}$ is sufficiently smooth, then we can do without $X$. This was explained in 3] by the example of the wave equation. Phenomena of this kind are well studied in the theory of elliptic problems (see, e.g., [17]).

\section{§8. ENERGY ESTIMATES FOR SOLUTIONS IN A BOUNDED DOMAIN}

Let $G$ be a domain in $\mathbb{R}^{m}$ with compact closure $\bar{G}$ and with boundary $\partial G$ containing an edge $\mathcal{M}$. Outside $\mathcal{M}$ the boundary is smooth. The edge $\mathcal{M}$ is a smooth closed $(m-n)$ dimensional submanifold in $\mathbb{R}^{m}$. For any point $z \in \mathcal{M}$ there exists a neighborhood $\mathcal{U} \subset \mathbb{R}^{m}$ and a diffeomorphism $\kappa: \mathcal{U} \rightarrow \kappa(\mathcal{U}) \subset \mathbb{R}^{m}$ such that $\kappa(z)=0, \kappa^{\prime}(z)=I$, and $\kappa(\mathcal{U} \cap G)=\mathbb{D} \cap \mathbb{B}$, where $\mathbb{B}$ is the open unit ball in $\mathbb{R}^{m}$ centered at the origin, and $\mathbb{D}=\mathbb{K} \times \mathbb{R}^{m-n} \subset \mathbb{R}^{m}$ is a wedge with edge $M$. The mapping $\kappa$ takes the points in $\mathcal{M} \subset \partial G$ to $M$. We say that the edge $\mathcal{M}$ is admissible if for any $z \in \mathcal{M}$ the corresponding edge $\mathbb{D}$ is admissible in the sense of Definition 3.1.1. The domains with admissible edge are needed only for a mixed estimate of solutions with nonhomogeneous 
Dirichlet condition. In all other cases we do not require that the edges be admissible; in other words, the edges satisfy all the above conditions, possibly except admissibility.

In the cylinder $G \times \mathbb{R}$, we consider the operators

$$
\begin{aligned}
\left(\mathcal{L}\left(x, D_{x}, D_{t}\right) u\right)_{i} & =\partial_{t}^{2} u_{i}(x, t)-\partial_{k}\left(a_{i j}^{k l}(x) \partial_{l} u_{j}(x, t)\right), \\
\left(\mathcal{B}\left(x, \partial_{x}\right) u\right)_{i} & =\nu_{k}(x) a_{i j}^{k l}(x) \partial_{l} u_{j}
\end{aligned}
$$

with coefficients $a_{i j}^{k l}$ belonging to $\mathcal{C}^{\infty}(\bar{G})$ and satisfying (16), (17) in $\bar{G}$; here $\nu(x)=$ $\left(\nu_{1}(x), \ldots, \nu_{m}(x)\right)$ stands for the outward unit normal at $x \in \partial G \backslash \mathcal{M}$.

In what follows we shall need Korn's inequality. If $\partial G$ is Lipschitz, then for $u$ in $H^{1}(G)$ we have the estimate

$$
\int_{G}\left|\nabla_{x} u(x)\right|^{2} \mathrm{~d} x \leq c\left\{\int_{G} \sum_{i, k}\left|\partial_{k} u_{i}(x)+\partial_{i} u_{k}(x)\right|^{2} \mathrm{~d} x+\int_{G}|u(x)|^{2} \mathrm{~d} x\right\}
$$

(see [6], §2, Theorem 2). Under our assumptions on $G$, the boundary $\partial G$ is not Lipschitz if the boundary $\partial \mathbb{D}$ of the wedge $\mathbb{D}$ in the definition of $G$ is not Lipschitz in a neighborhood of the edge $M$. The next lemma generalizes inequality (134) to such domains.

Lemma 8.1. Let $G$ be a domain with arbitrary edge $M$ satisfying the conditions given at the beginning of the section. Then estimate (134) is valid for the functions in $H^{1}(G)$.

Proof. Let $\mathcal{U}$ be a neighborhood of the edge $\mathcal{M}$ in $\mathbb{R}^{m}$, and let $\left\{U_{1}, \ldots, U_{J}\right\}$ be an open covering of $\mathcal{U}$ such that there exist diffeomorphisms $\kappa_{j}: U_{j} \rightarrow \mathbb{R}^{m}$ satisfying the conditions given in the above description of a domain with edge. Thus, $\kappa_{j}\left(U_{j} \cap G\right)=$ $\mathbb{B} \cap \mathbb{D}_{j}$, where $\mathbb{B}$ is an open ball in $\mathbb{R}^{m}$ and $\mathbb{D}_{j}=\mathbb{K}_{j} \times \mathbb{R}^{m-n}$ is an $m$-dimensional wedge, and there exists a point $z_{j} \in \mathcal{M} \cap U_{j}$ for which $\kappa_{j}\left(z_{j}\right)=0, \kappa_{j}^{\prime}\left(z_{j}\right)=I$.

Let $U_{-L}, \ldots, U_{-1}$ be open balls in $\mathbb{R}^{m}$ such that $U_{-q} \cap \mathcal{M}=\varnothing, q=1, \ldots, L$, and $\left\{U_{-L}, \ldots, U_{-1}, U_{1}, \ldots, U_{J}\right\}$ is a covering of $\partial G$. Assume that $U_{0}$ is an open subset of $G$, $\overline{U_{0}} \subset G$, and $\left\{U_{-L}, \ldots, U_{-1}, U_{0}, U_{1}, \ldots, U_{J}\right\}$ is a covering of $\bar{G}$. We introduce a partition of unity $\left\{\chi_{-L}, \ldots, \chi_{J}\right\}$ subordinate to the covering $\left\{U_{-L}, \ldots, U_{J}\right\}$.

Consider the integral

$$
\int_{U \cap G} \sum_{i, j}\left|\frac{\partial\left(\chi u_{i}\right)}{\partial x_{j}}+\frac{\partial\left(\chi u_{j}\right)}{\partial x_{i}}\right|^{2}
$$

where $(U, \kappa)$ is one of the charts $\left\{\left(U_{j}, \kappa_{j}\right)\right\}_{j=1}^{J}$ and $\chi$ is the corresponding function in the partition of unity. Changing the variables $x \mapsto y=\kappa(x)$, we rewrite the integral (135) in the form

$$
\int_{\mathbb{D}} \sum_{i, j}\left|\sum_{k} \frac{\partial y_{k}}{\partial x_{j}} \frac{\partial\left(\chi u_{i}\right)}{\partial y_{k}}+\sum_{k} \frac{\partial y_{k}}{\partial x_{i}} \frac{\partial\left(\chi u_{j}\right)}{\partial y_{k}}\right|^{2} \mathrm{~d} x .
$$

Since $\left(\kappa^{\prime}\right)^{-1}(0)=I$, we have $\partial y_{k} / \partial x_{j}=\delta_{k, j}+O(y)$. Therefore, assuming the covering to be sufficiently fine, we obtain

$$
\begin{aligned}
\int_{\mathbb{D}} \sum_{i, j}\left|\sum_{k} \frac{\partial y_{k}}{\partial x_{j}} \frac{\partial\left(\chi u_{i}\right)}{\partial y_{k}}+\sum_{k} \frac{\partial y_{k}}{\partial x_{i}} \frac{\partial\left(\chi u_{j}\right)}{\partial y_{k}}\right|^{2} \mathrm{~d} y \\
\quad \geq \int_{\mathbb{D}} \sum_{i, j}\left|\frac{\partial\left(\chi u_{i}\right)}{\partial y_{j}}+\frac{\partial\left(\chi u_{j}\right)}{\partial y_{i}}\right|^{2} \mathrm{~d} y-\varepsilon \int_{\mathbb{D}}\left|\nabla_{y}(\chi u(y))\right|^{2} \mathrm{~d} y \\
\quad \geq(c-\varepsilon) \int_{\mathbb{D}}\left|\nabla_{y}(\chi u(y))\right|^{2} \mathrm{~d} y
\end{aligned}
$$


at the last step we applied inequality (20). Consequently,

$$
\begin{aligned}
\int_{G} \mid & \left.\nabla_{x}(\chi u(x))\right|^{2} \mathrm{~d} x \leq c \int_{\mathbb{D}}\left|\nabla_{y}(\chi u(y))\right|^{2} \mathrm{~d} y \\
& \leq \int_{G \cap U} \sum_{i, j}\left|\frac{\partial\left(\chi u_{i}\right)}{\partial x_{j}}+\frac{\partial\left(\chi u_{j}\right)}{\partial x_{i}}\right|^{2} \mathrm{~d} x \\
& \leq \int_{G \cap U} \sum_{i, j}\left|\chi \frac{\partial u_{i}}{\partial x_{j}}+\chi \frac{\partial u_{j}}{\partial x_{i}}\right|^{2} \mathrm{~d} x+\int_{G}|u(x)|^{2} \mathrm{~d} x .
\end{aligned}
$$

Moreover, by (134),

$$
\int_{G \cap U_{k}}\left|\nabla_{x}\left(\chi_{k} u(x)\right)\right|^{2} \mathrm{~d} x \leq \int_{G \cap U_{k}} \sum_{i, j}\left|\chi_{k} \frac{\partial u_{i}}{\partial x_{j}}+\chi_{k} \frac{\partial u_{j}}{\partial x_{i}}\right|^{2} \mathrm{~d} x+\int_{G}|u(x)|^{2} \mathrm{~d} x,
$$

where $k=-L, \ldots, 0$. Using (136) and (137), we get

$$
\begin{gathered}
\int_{G}\left|\nabla_{x}(u(x))\right|^{2} \mathrm{~d} x \leq \sum_{j=-L}^{J} \int_{G \cap U_{j}}\left|\nabla_{x}\left(\chi_{j} u(x)\right)\right|^{2} \mathrm{~d} x \\
\leq\left\{\int_{G} \sum_{i, j}\left|\frac{\partial u_{i}}{\partial x_{j}}+\frac{\partial u_{j}}{\partial x_{i}}\right|^{2} \mathrm{~d} x+\int_{G}|u(x)|^{2} \mathrm{~d} x\right\} .
\end{gathered}
$$

In the domain $G$, we consider the Dirichlet problem operator

$$
u \mapsto\left\{\mathcal{L}\left(x, D_{x}, \tau\right) u,\left.u\right|_{G \backslash \mathcal{M}}\right\}
$$

and the Neumann problem operator

$$
u \mapsto\left\{\mathcal{L}\left(x, D_{x}, \tau\right) u,\left.\mathcal{B}\left(x, D_{x}\right) u\right|_{G \backslash \mathcal{M}}\right\},
$$

depending on the parameter $\tau \in \mathbb{C}$.

First, we estimate solutions of the Dirichlet problem with homogeneous boundary condition. We introduce the set

$$
\left\{v \in \mathcal{C}^{\infty}(\bar{G} \backslash \mathcal{M}) \cap H^{1}(G):\left.v\right|_{\partial G \backslash \mathcal{M}}=0, \mathcal{L}\left(x, D_{x}, \tau\right) v \in L_{2}(G)\right\} .
$$

Proposition 8.2. Let $G$ be a domain with edge $\mathcal{M}$ (we do not suppose that the edge is admissible). For the elements of the set (140), we have

$$
\gamma^{2} \int_{G}\left(|\tau|^{2}|v(x)|^{2}+\left|\nabla_{x} v(x)\right|^{2}\right) \mathrm{d} x \leq c \int_{G}\left|\mathcal{L}\left(x, D_{x}, \tau\right) v(x)\right|^{2} \mathrm{~d} x
$$

with a constant $c$ independent of $\tau=\sigma-i \gamma$, where $\sigma \in \mathbb{R}$ and $\gamma>0$.

The proof of the proposition is similar to that of Proposition 2.0.2, we use estimate (20) with $\mathbb{D}$ replaced by $G$.

Now, turning to the Neumann problem with homogeneous boundary condition, we introduce the set

$$
\left\{v \in \mathcal{C}^{\infty}(\bar{G} \backslash \mathcal{M}) \cap H^{1}(G):\left.\mathcal{B}\left(x, D_{x}\right) v\right|_{\partial G \backslash \mathcal{M}}=0, \mathcal{L}\left(x, D_{x}, \tau\right) v \in L_{2}(G)\right\} .
$$


Proposition 8.3. Let $G$ be a domain with edge $\mathcal{M}$ (the edge may fail to be admissible). For the elements of the set (142), we have

$$
\gamma^{2} \int_{G}\left(|\tau|^{2}|v(x)|^{2}+\left|\nabla_{x} v(x)\right|^{2}\right) \mathrm{d} x \leq c \int_{G}\left|\mathcal{L}\left(x, D_{x}, \tau\right) v(x)\right|^{2} \mathrm{~d} x
$$

with a constant $c$ independent of $\tau=\sigma-i \gamma$, where $\sigma \in \mathbb{R}, \gamma>\gamma_{0}$, and $\gamma_{0}$ is a sufficiently large positive number.

Proof. We can use the argument in the proof of Proposition 2.0.2, employing inequality (134) instead of (20). The additional term in (134) (in comparison with (20)) forces us to choose a sufficiently large $\gamma$.

We pass to the Dirichlet and Neumann problems in $G$ with nonhomogeneous boundary conditions. Consider the Dirichlet problem operator on the set

$$
\left\{v \in \mathcal{C}^{\infty}(\bar{G} \backslash M) \cap H^{1}(G): \mathcal{L}\left(x, D_{x}, \tau\right) v \in L_{2}(G),\left.v\right|_{\partial G} \in H^{1}(\partial G)\right\} .
$$

Proposition 8.4. Let $G$ be a domain with admissible edge $\mathcal{M}$. For the elements of the set (144), we have

$$
\begin{aligned}
& \gamma^{2} \int_{G}\left(\left|\nabla_{x} u(x)\right|^{2}+|\tau|^{2}|v(x)|^{2}\right) \mathrm{d} x+\gamma \int_{\partial G}\left|\partial_{\nu} u\right|^{2} \mathrm{~d} S \\
& \quad \leq c\left\{\int_{G}\left|\mathcal{L}\left(x, D_{x}, \tau\right) u(x)\right|^{2} \mathrm{~d} x+\gamma \int_{\partial G}\left(|\tau|^{2}|u(x)|^{2}+\left|\nabla_{\sigma} u(x)\right|^{2}\right) \mathrm{d} S\right\},
\end{aligned}
$$

where $\nabla_{\sigma}$ stands for the tangent gradient on $\partial G \backslash \mathcal{M}$. The constant $c$ is independent of $\tau=\sigma-i \gamma, \sigma \in \mathbb{R}, \gamma \geq \gamma_{0}$, and $\gamma_{0}$ is a sufficiently large positive number.

Proof. Let $\left\{U_{j}\right\}_{j=-L}^{J}$ be the same covering as in Lemma 8.1 and let $\left\{\chi_{j}\right\}_{j=-L}^{J}$ be a partition of unity subordinate to the covering. We assume that the covering is sufficiently fine. For any $q=-L, \ldots,-1,1, \ldots, J$ we can choose a constant vector $l^{(q)}=\left(l_{1}^{(q)}, \ldots, l_{m}^{(q)}\right)$ satisfying $\left\langle l^{(q)}, \nu(x)\right\rangle \geq c>0$ for $x \in \partial G \cap U_{q}$, where $\nu(x)=\left(\nu_{1}(x), \ldots, \nu_{m}(x)\right)$ is the outward unit normal to $\partial G \backslash \mathcal{M}$. Such a choice for $q=1, \ldots, J$ is possible because $\mathcal{M}$ is admissible. For the role of $u$ in (46) we take $\chi_{q} u$ and put $\left\{f^{\alpha}\right\}_{\alpha=1}^{m+1}=\left\{l_{1}^{(q)}, \ldots, l_{m}^{(q)}, l_{m+1}^{(q)}\right\}$ with positive $l_{m+1}^{(q)}$ such that $l_{m+1}^{(q)}>c\left|l^{(q)}\right|$ and $c$ is sufficiently large (cf. (54)). Integrating the resulting formula over $G$ and arguing as in the proof of Proposition 3.1.2, we arrive at the estimate

$$
\begin{aligned}
& \gamma^{2} \int_{G}\left(\left|\nabla_{x}\left(\chi_{q}(x) u(x)\right)\right|^{2}+|\tau|^{2}\left|\chi_{q}(x) u(x)\right|^{2}\right) \mathrm{d} x \\
& +\gamma \int_{\partial G} \sum_{i, k}\left|\partial_{\nu}\left(\chi_{q} u_{i}\right) \nu_{k}+\partial_{\nu}\left(\chi_{q} u_{k}\right) \nu_{i}\right|^{2} \mathrm{~d} S \\
& \leq c \\
& \qquad \int_{G}\left|\mathcal{L}\left(x, D_{x}, \tau\right)\left(\chi_{q}(x) u(x)\right)\right|^{2} \mathrm{~d} x \\
& \left.\quad+\gamma \int_{\partial G}\left(|\tau|^{2}\left|\chi_{q}(x) u(x)\right|^{2}+\left|\nabla_{\sigma}\left(\chi_{q}(x) u(x)\right)\right|^{2}\right) \mathrm{d} S\right\} .
\end{aligned}
$$


The function $\chi_{0} u$ can be majorized with the help of (141). Furthermore,

$$
\begin{aligned}
\gamma^{2} \int_{G}\left(\left|\nabla_{x} u(x)\right|^{2}+|\tau|^{2}|u(x)|^{2}\right) \mathrm{d} x+\gamma \int_{\partial G} \sum_{i, k}\left|\partial_{\nu} u_{i} \nu_{k}+\partial_{\nu} u_{k} \nu_{i}\right|^{2} \mathrm{~d} S \\
\leq \sum_{q=-L}^{J} \gamma^{2} \int_{G}\left(\left|\nabla_{x}\left(\chi_{q}(x) u(x)\right)\right|^{2}+|\tau|^{2}\left|\chi_{q}(x) u(x)\right|^{2}\right) \mathrm{d} x \\
\quad+\sum_{q=-L, q \neq 0}^{J} \gamma \int_{\partial G} \sum_{i, k}\left|\partial_{\nu}\left(\chi_{q}(x) u_{i}(x)\right) \nu_{k}+\partial_{\nu}\left(\chi_{q}(x) u_{k}(x)\right) \nu_{i}\right|^{2} \mathrm{~d} S \\
\leq\left\{\sum_{q=-L}^{J} \int_{G}\left|\mathcal{L}\left(x, D_{x}, \tau\right)\left(\chi_{q}(x) u(x)\right)\right|^{2} \mathrm{~d} x\right. \\
\left.\quad+\sum_{q=-L, q \neq 0}^{J} \gamma \int_{\partial G}\left(|\tau|^{2}\left|\chi_{q}(x) u(x)\right|^{2}+\left|\nabla_{\sigma}\left(\chi_{q}(x) u(x)\right)\right|^{2}\right) \mathrm{d} S\right\} .
\end{aligned}
$$

Since $\mathcal{L} \chi_{q} u=\chi_{q} \mathcal{L} u+\left[\mathcal{L}, \chi_{q}\right] u$, we have

$$
\left\|\mathcal{L}\left(\chi_{q} u\right) ; L_{2}(G)\right\| \leq\left\|\chi_{q} \mathcal{L} u ; L_{2}(G)\right\|+\left\|u ; H^{1}(G)\right\| .
$$

Similarly,

$$
\left\|\nabla \sigma\left(\chi_{q} u\right) ; L_{2}(\partial G)\right\| \leq\left\|\chi_{q} \nabla_{\sigma} u ; L_{2}(\partial G)\right\|+\left\|u ; L_{2}(\partial G)\right\| .
$$

This and Lemma 3.1.4 lead to an inequality of the form (145) with the additional term $\left\|u ; H^{1}(G)\right\|$ on the right-hand side. The term can be dropped for large $\gamma$.

The Neumann problem operator will be considered on the set

$$
\begin{aligned}
& \left\{v \in \mathcal{C}^{\infty}(\bar{G} \backslash \mathcal{M}) \cap H^{1}(\mathbb{D}):\right. \\
& \left.\quad \mathcal{B}\left(x, D_{x}\right) v \in H^{-1 / 2}(\partial G), \mathcal{L}\left(x, D_{x}, \tau\right) v \in L_{2}(G)\right\} .
\end{aligned}
$$

Proposition 8.5. Let $G$ be a domain with edge $\mathcal{M}$ (which may fail to be admissible). For the functions in the set (146), we have

$$
\begin{aligned}
& \gamma^{2} \int_{G}\left(\left|\nabla_{x} v(x)\right|^{2}+|\tau|^{2} \cdot|v(x)|^{2}\right) \mathrm{d} x \\
& \quad \leq c\left\{\int_{G}\left|\mathcal{L}\left(x, D_{x}, \tau\right) v(x)\right|^{2} \mathrm{~d} x+|\tau|^{2}\left\|\mathcal{B}\left(x, D_{x}\right) v ; H^{-1 / 2}(\partial G)\right\|^{2}\right\}
\end{aligned}
$$

with constant $c$ independent of $\tau=\sigma-i \gamma$, where $\sigma \in \mathbb{R}, \gamma>\gamma_{0}$, and $\gamma_{0}$ is a sufficiently large positive number.

Proof. Employing inequality (134) instead of (20), we can repeat the arguments used in the proof of Propositions 3.2.1 and 3.2.2.

Remark 8.6. We considered the cases of $n>2$ and $n=2$ for the wedge $\mathbb{D}$ separately (see Propositions 3.2.1 and 3.2.2). The reason was that, for $n>2$, the estimate was obtained for $\gamma>0$, while for $n=2$ it was proved only for $\gamma>\gamma_{0}>0$ with sufficiently large $\gamma_{0}$. Since inequality (134) involves the additional term $\left\|u ; L_{2}\right\|$ (in comparison with (20)), we are forced to take large $\gamma$ for $n>2$ as well. Therefore, the class $H_{0}^{-1 / 2}(\partial G)$ gives no advantage, and both cases where $n=\operatorname{codim} \mathcal{M}=2$ and $n=\operatorname{codim} \mathcal{M}>2$ are treated together. 


\section{§9. Weighted EStimates In A BOUNDED DOMAIN With EDGE}

To explain the proof avoiding minor technicalities, we first consider a domain $G \in$ $\mathbb{R}^{n}$ with conical point $\mathcal{O}$. Moreover, we suppose that $G$ coincides with a cone in a neighborhood of $\mathcal{O}$.

The spaces $H_{\beta}^{s}(\mathbb{D})$ and $H_{\beta}^{s}(\mathbb{D}, q)$ were introduced at the beginning of $\S 5$. Replacing $r$ with $\operatorname{dist}(x, \mathcal{M})$ and $\mathbb{D}$ with $G$ in the expressions for the norms of $H_{\beta}^{s}(\mathbb{D})$ and $H_{\beta}^{s}(\mathbb{D}, q)$, we obtain the norms of $H_{\beta}^{s}(G)$ and $H_{\beta}^{s}(G, q)$. We start with the Dirichlet problem. Consider the set

$$
\left\{u \in H_{\beta}^{2}(G): \mathcal{L}\left(x, D_{x}, \tau\right) u \in L_{2}(G),\left.u\right|_{\partial G} \in H^{1}(\partial G)\right\} .
$$

Proposition 9.1. Let $G$ be a domain that coincides with an admissible cone $\mathbb{K}$ in $a$ neighborhood of the point $\mathcal{O}$. Assume that $\beta \leq 1$ and that the line $\{\lambda \in \mathbb{C}: \operatorname{Im} \lambda=$ $\beta+n / 2-2\}$ contains no eigenvalues of the pencil $\mathfrak{A}_{D}$. Then for the functions in the set (148) we have

$$
\begin{aligned}
\gamma\left\|\partial_{\nu} u ; H_{\beta}^{0}(\partial G)\right\|^{2}+\gamma^{2}\left\|u ; H_{\beta}^{1}(G,|\tau|)\right\|^{2}+\left\|\xi_{\tau} u ; H_{\beta}^{2}(G,|\tau|)\right\|^{2} \\
\leq c\left\{\left\|\mathcal{L}\left(x, D_{x}, \tau\right) u ; H_{\beta}^{0}(G)\right\|^{2}+\left\|\xi_{\tau} u ; H_{\beta}^{3 / 2}(\partial G)\right\|^{2}+\gamma\left\|u ; H_{\beta}^{1}(\partial G,|\tau|)\right\|^{2}\right. \\
+\left(|\tau|^{2(1-\beta)} / \gamma^{2}+1\right) \\
\left.\quad \times\left(\left\|\mathcal{L}\left(x, D_{x}, \tau\right) u ; L_{2}(G)\right\|^{2}+\gamma\left\|\nabla_{\sigma} u ; L_{2}(\partial G)\right\|^{2}+\gamma|\tau|^{2}\left\|u ; L_{2}(\partial G)\right\|^{2}\right)\right\},
\end{aligned}
$$

where $\xi_{\tau}(x)=\xi(|\tau| x)$ for $|x|<\delta /|\tau|$ and $\xi_{\tau}(x)=0$ for all $x \in G$ such that $|x|>$ $\delta /|\tau|$; here $\xi$ stands for a cut-off function in $\mathcal{C}^{\infty}(\overline{\mathbb{D}})$ satisfying $\xi(x)=0$ for $|x|>\delta$ and $\xi(x)=1$ for $|x|<\delta / 2$, with a sufficiently small positive $\delta$. If in (148) the requirement $\left.u\right|_{\partial G} \in H^{1}(\partial G)$ is replaced by the condition $\left.u\right|_{\partial G}=0$, then

$$
\begin{aligned}
& \gamma^{2}\left\|u ; H_{\beta}^{1}(G,|\tau|)\right\|^{2}+\left\|\xi_{\tau} u ; H_{\beta}^{2}(G,|\tau|)\right\|^{2} \\
& \quad \leq c\left\{\left\|\mathcal{L}\left(x, D_{x}, \tau\right) u ; H_{\beta}^{0}(G)\right\|^{2}+\left(\frac{|\tau|^{2(1-\beta)}}{\gamma^{2}}+1\right)\left\|\mathcal{L}\left(x, D_{x}, \tau\right) u ; L_{2}(G)\right\|^{2}\right\}
\end{aligned}
$$

for any cone $\mathbb{K}$ (which may fail to be admissible). The constants $c$ in the inequalities are independent of $\tau=\sigma-i \gamma$ for $\sigma \in \mathbb{R}$ and $\gamma>\gamma_{0}>0$ with sufficiently large $\gamma_{0}$.

Proof. Let $\mathcal{L}_{0}$ denote the operator obtained by freezing the coefficients of $\mathcal{L}$ at the conical point. We have

$$
\mathcal{L}_{0}\left(\xi_{\tau} u\right)=\left(\mathcal{L}-\mathcal{L}_{0}\right) \xi_{\tau} u+\left[\mathcal{L}, \xi_{\tau}\right] u+\xi_{\tau} \mathcal{L} u
$$

We can view (151) as an equation in $\mathbb{K}$ and use inequality (93) with $u$ and $\mathcal{L}\left(D_{x}, \tau\right) u$ replaced by $\xi_{\tau} u$ and the right-hand side of (151), respectively. Application of the readymixed estimate (89) instead of the intermediate inequality (93) would lead to more cumbersome calculations in the remaining part of the proof. Assume that the function $\chi$ in (93) is chosen so that $\chi \xi=\xi$. Then, by (93) and (151), we have

$$
\begin{aligned}
\gamma \| \partial_{\nu} u ; & H_{\beta}^{0}(\partial G)\left\|^{2}+\gamma^{2}\right\| u ; H_{\beta}^{1}(G,|\tau|)\left\|^{2}+\right\| \xi_{\tau} u ; H_{\beta}^{2}(G,|\tau|) \|^{2} \\
\leq c & \left\|\left(\mathcal{L}_{0}-\mathcal{L}\right) \xi_{\tau} u ; H_{\beta}^{0}(G)\right\|^{2}+\left\|\left[\mathcal{L}, \xi_{\tau}\right] u ; H_{\beta}^{0}(G)\right\|^{2}+\left\|\xi_{\tau} \mathcal{L} u ; H_{\beta}^{0}(G)\right\|^{2} \\
\quad+\left\|\xi_{\tau} u ; H_{\beta}^{3 / 2}(\partial G)\right\|^{2} & \left.\quad+\gamma\left\|\psi_{\infty, \tau} \xi_{\tau} u ; H_{\beta}^{1}(\partial G,|\tau|)\right\|^{2}+\left\|\psi_{\infty, \tau} \xi_{\tau} u ; H_{\beta-1}^{1}(G)\right\|^{2}+|\tau|^{2}\left\|\xi_{\tau} u ; H_{\beta}^{1}(G,|\tau|)\right\|^{2}\right\} .
\end{aligned}
$$


Consider the first term on the right. Since the support of $\xi_{\tau}$ is small and the coefficients of $\mathcal{L}$ are continuous, we obtain

$$
\left\|\left(\mathcal{L}_{0}-\mathcal{L}\right) \xi_{\tau} u ; H_{\beta}^{0}(G)\right\|^{2} \leq \varepsilon\left\|\xi_{\tau} u ; H_{\beta}^{2}(G)\right\|^{2} .
$$

Furthermore,

$$
\begin{aligned}
& \left\|\left[\mathcal{L}, \xi_{\tau}\right] u ; H_{\beta}^{0}(G)\right\|^{2} \leq\left\|\left|\nabla \xi_{\tau}\right| \nabla u ; H_{\beta}^{0}(G)\right\|^{2} \\
& \quad \leq c|\tau|^{2} \int_{a<|\tau| r<b}|\nabla u|^{2} r^{2 \beta} \mathrm{d} x \leq c|\tau|^{2(1-\beta)} \int_{G}|\nabla u|^{2} \mathrm{~d} x .
\end{aligned}
$$

Thus,

$$
\begin{aligned}
& \left\|\mathcal{L}_{0}\left(\xi_{\tau} u\right) ; H_{\beta}^{0}(G)\right\|^{2} \\
& \quad \leq c\left\|\xi_{\tau} \mathcal{L} u ; H_{\beta}^{0}(G)\right\|^{2}+\varepsilon\left\|\xi_{\tau} u ; H_{\beta}^{2}(G)\right\|^{2}+c|\tau|^{2(1-\beta)} \int_{G}|\nabla u|^{2} \mathrm{~d} x .
\end{aligned}
$$

In essence, the next two terms on the right in (152) occur on the right-hand side of (149). Turning to the two last terms, we have

$$
\begin{aligned}
& \left\|\psi_{\infty, \tau} \xi_{\tau} u ; H_{\beta-1}^{1}(G)\right\|^{2} \leq c \int_{a<|\tau| r<b} r^{2(\beta-1)}\left(|\nabla u|^{2}+|\tau|^{2}+r^{-2}|u|^{2}\right) \mathrm{d} x \\
& \quad \leq c|\tau|^{2(1-\beta)}\left(\left\|\nabla u ; L_{2}(G)\right\|^{2}+|\tau|^{2}\left\|u ; L_{2}(G)\right\|^{2}\right) .
\end{aligned}
$$

Finally,

$$
\begin{aligned}
|\tau|^{2} & \left\|\xi_{\tau} u ; H_{\beta}^{1}(G,|\tau|)\right\|^{2} \\
\leq & c|\tau|^{2}\left(\int_{r|\tau|<\varepsilon}+\int_{\varepsilon<r|\tau|<b}\right) r^{2 \beta}\left(|\nabla u|^{2}+r^{-2}|u|^{2}+|\tau|^{2}|u|^{2}\right) \mathrm{d} x \\
\leq & \varepsilon^{2} \int_{r|\tau|<\varepsilon} r^{2(\beta-1)}\left(|\nabla u|^{2}+r^{-2}|u|^{2}+|\tau|^{2}|u|^{2}\right) \mathrm{d} x \\
\quad & \quad+|\tau|^{2(1-\beta)} \int_{\varepsilon<r|\tau|<b}\left(|\nabla u|^{2}+|\tau|^{2}|u|^{2}\right) \mathrm{d} x \\
\leq & \varepsilon^{2}\left\|\xi_{\tau} u ; H_{\beta}^{2}(G,|\tau|)\right\|^{2}+c|\tau|^{2(1-\beta)}\left(|| \nabla u ; L_{2}(G)\left\|^{2}+|\tau|^{2}\right\| u ; L_{2}(G) \|^{2}\right) .
\end{aligned}
$$

Combining (152)-(155), we arrive at the inequality

$$
\begin{aligned}
\gamma \| \partial_{\nu} u ; & H_{\beta}^{0}(\partial G)\left\|^{2}+\gamma^{2}\right\| u ; H_{\beta}^{1}(G,|\tau|)\left\|^{2}+\right\| \xi_{\tau} u ; H_{\beta}^{2}(G,|\tau|) \|^{2} \\
\leq c\{ & \left\|\mathcal{L}\left(x, D_{x}, \tau\right) u ; H_{\beta}^{0}(G)\right\|^{2}+\left\|\xi_{\tau} u ; H_{\beta}^{3 / 2}(\partial G)\right\|^{2} \\
& +\gamma\left\|u ; H_{\beta}^{1}(\partial G,|\tau|)\right\|^{2}+\varepsilon^{2}\left\|\xi_{\tau} u ; H_{\beta}^{2}(G)\right\|^{2} \\
& \left.+|\tau|^{2(1-\beta)}\left(\left\|\nabla u ; L_{2}(G)\right\|^{2}+|\tau|^{2}\left\|u ; L_{2}(G)\right\|^{2}\right)\right\} .
\end{aligned}
$$

It remains to estimate $\gamma^{2}\left\|\left(1-\xi_{\tau}\right) u ; H_{\beta}^{1}(G,|\tau|)\right\|^{2}$ and $\gamma\left\|\left(1-\xi_{\tau}\right) \partial_{\nu} u ; H_{\beta}^{0}(\partial G)\right\|^{2}$. We have

$$
\begin{aligned}
& \gamma^{2}\left\|\left(1-\xi_{\tau}\right) u ; H_{\beta}^{1}(G,|\tau|)\right\|^{2} \\
& \leq \gamma^{2}\left(\int_{a<r|\tau|} r^{2 \beta}\left(|\nabla u|^{2}+|\tau|^{2}|u|^{2}\right) \mathrm{d} x\right. \\
& \left.\quad+|\tau|^{2} \int_{a<r|\tau|<b} r^{2 \beta}|u|^{2} \mathrm{~d} x+\int_{a<r|\tau|} r^{2(\beta-1)}|u|^{2} \mathrm{~d} x\right) \\
& \quad \leq c \gamma^{2} \int_{a<r|\tau|} r^{2 \beta}\left(|\nabla u|^{2}+|\tau|^{2}|u|^{2}\right) \mathrm{d} x+\gamma^{2}|\tau|^{2(1-\beta)} \int_{G}|u|^{2} \mathrm{~d} x .
\end{aligned}
$$


The first term is estimated differently for $\beta \geq 0$ and $\beta<0$. If $\beta \geq 0$, then $r^{2 \beta} \leq c$ because the domain $G$ is bounded. For $\beta<0$, we clearly have $r^{2 \beta}<c|\tau|^{-2 \beta}$. Denoting by $W$ the right-hand side of (145), we arrive at the inequality

$$
\gamma^{2}\left\|\left(1-\xi_{\tau}\right) u ; H_{\beta}^{1}(G,|\tau|)\right\|^{2} \leq c\left(1+|\tau|^{-2 \beta}\right) W \leq c\left(1+\frac{|\tau|^{2(1-\beta)}}{\gamma^{2}}\right) W .
$$

The expression $\gamma\left\|\left(1-\xi_{\tau}\right) \partial_{\nu} u ; H_{\beta}^{0}(\partial G)\right\|^{2}$ can be estimated in a similar way. From (156), (157), and (145) we deduce the estimate

$$
\begin{aligned}
& \gamma\left\|\partial_{\nu} u ; H_{\beta}^{0}(\partial G)\right\|^{2}+\gamma^{2}\left\|u ; H_{\beta}^{1}(G,|\tau|)\right\|^{2}+\left\|\xi_{\tau} u ; H_{\beta}^{2}(G,|\tau|)\right\|^{2} \\
& \leq c\{\left\|\mathcal{L}\left(x, D_{x}, \tau\right) u ; H_{\beta}^{0}(G)\right\|^{2}+\left\|\xi_{\tau} u ; H_{\beta}^{3 / 2}(\partial G)\right\|^{2} \\
&\left.\quad+\gamma\left\|u ; H_{\beta}^{1}(\partial G,|\tau|)\right\|^{2}+\left(|\tau|^{2(1-\beta)}+1\right) W+\varepsilon^{2}\left\|\xi_{\tau} u ; H_{\beta}^{2}(G)\right\|^{2}\right\} .
\end{aligned}
$$

Since $\varepsilon$ is a small number, we can $\operatorname{drop} \varepsilon^{2}\left\|\xi_{\tau} u ; H_{\beta}^{2}(G)\right\|^{2}$, replacing $c$ with a larger constant.

The next assertion contains an estimate of the solutions of the Neumann problem; we restrict ourselves to the case where $\operatorname{dim} G=n>2$. The nonhomogeneous boundary condition requires no additional assumptions on the domain $G$ in comparison with the homogeneous condition.

Proposition 9.2. Suppose a domain $G$ coincides with a cone $\mathbb{K}$ in a neighborhood of the point $\mathcal{O}$. Assume that $\beta \leq 1$ and that the line $\{\lambda \in \mathbb{C}: \operatorname{Im} \lambda=\beta+n / 2-2\}$ contains no eigenvalues of the pencil $\mathfrak{A}_{N}$. If $v \in H_{\beta}^{2}(G)$ is such that $\mathcal{L}\left(x, D_{x}, \tau\right) v \in L_{2}(G)$, then (158)

$$
\begin{aligned}
\gamma^{2}(\| \nabla u ; & \left.H_{\beta}^{0}(G)\left\|^{2}+|\tau|^{2}\right\| u ; H_{\beta}^{0}(G) \|^{2}\right)+\left\|\xi_{\tau} u ; H_{\beta}^{2}(G,|\tau|)\right\|^{2} \\
\leq c\{ & \left\|\mathcal{L}\left(x, D_{x}, \tau\right) u ; H_{\beta}^{0}(G)\right\|^{2}+\left\|\xi_{\tau} \mathcal{B}\left(x, D_{x}\right) u ; H_{\beta}^{1 / 2}(\partial G)\right\|^{2} \\
& +|\tau|^{2}\left\|\mathcal{B}\left(x, D_{x}\right) u ; H_{\beta+1 / 2}^{0}(\partial G)\right\|^{2} \\
& \left.+\left(\frac{|\tau|^{2(1-\beta)}}{\gamma^{2}}+1\right)\left(\left\|\mathcal{L}\left(x, D_{x}, \tau\right) u ; L_{2}(G)\right\|^{2}+|\tau|^{2}\left\|\mathcal{B}\left(x, D_{x}\right) u ; L_{2}(\partial G)\right\|^{2}\right)\right\}
\end{aligned}
$$

with a constant $c$ independent of $\tau=\sigma-i \gamma$, where $\sigma \in \mathbb{R}, \gamma \geq \gamma_{0}>0$, and $\gamma_{0}$ is a sufficiently large number.

This can be proved by a slight modification of the proof of Proposition 9.1. The starting point is the intermediate estimates (99) and (107) in Propositions 5.2.1 and 5.3 .3

We pass to estimates in a domain $G$ with edge $\mathcal{M}$ of codimension $\operatorname{codim} \mathcal{M}=n$. The opening of the tangent cone varies along the edge, and the pencil $\mathbb{C} \ni \lambda \mapsto \mathfrak{A}(\lambda, z)$, $z \in \mathcal{M}$, also varies. It may happen that for any number $\beta$ the line $\{\lambda \in \mathbb{C}: \operatorname{Im} \lambda=$ $\beta+n / 2-2\}$ contains points of the spectrum of the pencil $\mathfrak{A}(\cdot, z)$ for some $z \in \mathcal{M}$. Then, for any $\beta \in \mathbb{R}$, no elliptic estimate is possible in $H_{\beta}^{2}(G)$. Thus, we are forced to deal with a function $\mathcal{M} \ni z \mapsto \beta(z) \in \mathbb{R}$ and the spaces $H_{\beta(\cdot)}^{s}(G)$. Elliptic estimates in such a situation were described, e.g., in [15] or [17]. In fact, no new difficulties arise. So, to simplify the formulations, we assume that there exists $\beta \in \mathbb{R}$ such that the line $\{\lambda \in \mathbb{C}: \operatorname{Im} \lambda=\beta+n / 2-2\}$ is free from the eigenvalues of the pencil $\mathfrak{A}(\cdot, z)$ for all $z \in \mathcal{M}$.

Let $\left\{\left(U_{j}, \kappa_{j}\right)\right\}$ be a collection of charts in the definition of $G$ that cover a neighborhood of the edge $\mathcal{M}, \kappa_{j}: U_{j} \cap G \rightarrow \mathbb{D}_{j} \cap \mathbb{B}$. In the wedge $\mathbb{D}_{j}$, we consider a function $x=(y, z) \mapsto$ $\xi^{(j)}(y, z)$ that belongs to $\mathcal{C}^{\infty}\left(\overline{\mathbb{D}}_{j}\right)$, is independent of $z$, and is equal to 1 for $|y|<\delta$ and 
to 0 for $|y|>2 \delta$, where $\delta$ is a sufficiently small number. Put $\xi_{\tau}^{(j)}(y, z)=\xi^{(j)}(|\tau| y, z)$. Gluing the functions $\xi_{\tau}^{(j)}$ together by using a partition of unity, we define a function $\xi_{\tau}$ in $G$. The following proposition contains an estimate of solutions with Dirichlet boundary condition.

Proposition 9.3. Let $G$ be a domain with admissible edge $\mathcal{M}$. Suppose that $\beta \leq 1$ and that the line $\{\lambda \in \mathbb{C}: \operatorname{Im} \lambda=\beta+n / 2-2\}$ is free of the spectrum of the pencil $\mathfrak{A}_{D}(\cdot, z)$ for all $z \in \mathcal{M}$. Then for the functions in the set (148) we have

$$
\begin{aligned}
\gamma^{2}\left\|u ; H_{\beta}^{1}(G,|\tau|)\right\|^{2}+\left\|\xi_{\tau} u ; H_{\beta}^{2}(G,|\tau|)\right\|^{2} \\
\leq c\left\{\left\|\mathcal{L}\left(x, D_{x}, \tau\right) u ; H_{\beta}^{0}(G)\right\|^{2}+\left\|\xi_{\tau} u ; H_{\beta}^{3 / 2}(\partial G)\right\|^{2}+\gamma\left\|u ; H_{\beta}^{1}(\partial G,|\tau|)\right\|^{2}\right. \\
\quad+\left(\frac{|\tau|^{2(1-\beta)}}{\gamma^{2}}+1\right) \\
\left.\quad \times\left(\left\|\mathcal{L}\left(x, D_{x}, \tau\right) u ; L_{2}(G)\right\|^{2}+\gamma\left\|\nabla_{\sigma} u ; L_{2}(\partial G)\right\|^{2}+\gamma|\tau|^{2}\left\|u ; L_{2}(\partial G)\right\|^{2}\right)\right\},
\end{aligned}
$$

where $\xi_{\tau}(x)=\xi(|\tau| x)$ for $|x|<\delta /|\tau|$ and $\xi_{\tau}(x)=0$ for all $x \in G$ such that $|x|>\delta /|\tau|$, and $\xi$ stands for a cut-off function in $\mathcal{C}^{\infty}(\bar{G})$ satisfying $\xi(x)=0$ for $|x|>\delta$ and $\xi(x)=1$ for $|x|<\delta / 2$ with sufficiently small positive $\delta$.

If in (148) the requirement $\left.u\right|_{\partial G} \in H^{1}(\partial G)$ is replaced by the condition $\left.u\right|_{\partial G}=0$, then

$$
\begin{aligned}
& \gamma^{2}\left\|u ; H_{\beta}^{1}(G,|\tau|)\right\|^{2}+\left\|\xi_{\tau} u ; H_{\beta}^{2}(G,|\tau|)\right\|^{2} \\
& \quad \leq c\left\{\left\|\mathcal{L}\left(x, D_{x}, \tau\right) u ; H_{\beta}^{0}(G)\right\|^{2}+\left(\frac{|\tau|^{2(1-\beta)}}{\gamma^{2}}+1\right)\left\|\mathcal{L}\left(x, D_{x}, \tau\right) u ; L_{2}(G)\right\|^{2}\right\}
\end{aligned}
$$

for a domain with an arbitrary edge. The constants $c$ in (159) and (160) are independent of $\tau=\sigma-i \gamma$ for $\sigma \in \mathbb{R}$ and $\gamma>\gamma_{0}>0$, where $\gamma_{0}$ is a sufficiently large number.

We outline the proof. Let $\left(U_{j}, \kappa_{j}\right)$ be a chart in a neighborhood of the edge. Via the straightening diffeomorphism $\kappa_{j}$, we obtain a problem with variable coefficients in the wedge $\mathbb{D}_{j}$. After that, in essence, we repeat the argument used in the proof of Proposition 9.1. In particular, first we apply the intermediate inequality (93) to the map $(y, z) \mapsto \eta(z) \xi_{\tau}^{(j)}(y, z) u(y, z)$, where $\eta \in \mathcal{C}_{c}^{\infty}\left(\mathbb{R}^{m-n}\right), \eta=1$ near the origin and $\eta=0$ off a small neighborhood of the origin. In comparison with Proposition 9.1, some additional terms arise because of differentiation of the cut-off functions in $z$. This differentiation does not increase the order of growth in $|\tau|$. Therefore, choosing a sufficiently large constant $\gamma_{0}$, we can remove the terms mentioned above from the estimate.

In conclusion, we formulate an estimate of solutions with Neumann boundary condition for the case where $\mathcal{M}=n>2$.

Proposition 9.4. Let $G$ be a domain with edge $\mathcal{M}$. Assume that $\beta \leq 1$ and that the line $\{\lambda \in \mathbb{C}: \operatorname{Im} \lambda=\beta+n / 2-2\}$ is free of the spectrum of the pencil $\mathfrak{A}_{N}(\cdot, z)$ for all $z \in \mathcal{M}$. Then for the functions $v \in H_{\beta}^{2}(G)$ such that $\mathcal{L}\left(x, D_{x}, \tau\right) v \in L_{2}(G)$ we have (161)

$$
\begin{aligned}
& \gamma^{2}(\| \nabla u\left.; H_{\beta}^{0}(G)\left\|^{2}+|\tau|^{2}\right\| u ; H_{\beta}^{0}(G) \|^{2}\right)+\left\|\xi_{\tau} u ; H_{\beta}^{2}(G,|\tau|)\right\|^{2} \\
& \leq c\left\{\left\|\mathcal{L}\left(x, D_{x}, \tau\right) u ; H_{\beta}^{0}(G)\right\|^{2}+\left\|\xi_{\tau} \mathcal{B}\left(x, D_{x}\right) u ; H_{\beta}^{1 / 2}(\partial G)\right\|^{2}\right. \\
& \quad+|\tau|^{2}\left\|\mathcal{B}\left(x, D_{x}\right) u ; H_{\beta+1 / 2}^{0}(\partial G)\right\|^{2} \\
&\left.\quad+\left(\frac{|\tau|^{2(1-\beta)}}{\gamma^{2}}+1\right)\left(\left\|\mathcal{L}\left(x, D_{x}, \tau\right) u ; L_{2}(G)\right\|^{2}+|\tau|^{2}\left\|\mathcal{B}\left(x, D_{x}\right) u ; L_{2}(\partial G)\right\|^{2}\right)\right\}
\end{aligned}
$$

with the same $\xi_{\tau}$ as in (159). The constant $c$ is independent of $\tau=\sigma-i \gamma$ for $\sigma \in \mathbb{R}$ and $\gamma \geq \gamma_{0}>0$, where $\gamma_{0}$ is a sufficiently large number. 


\section{REFERENCES}

[1] B. A. Plamenevskiı̌, On the Dirichlet problem for the wave equation in a cylinder with edges, Algebra i Analiz 10 (1998), no. 2, 197-228 (Erratum: 10 (1998), no. 3, 224); English transl., St. Petersburg Math. J. 10 (1999), no. 2, 373-397. MR1629407 (99i:35087a) MR1628054(99i:35087b)

[2] A. Yu. Kokotov and B. A. Plamenevskiǐ, On the Cauchy-Dirichlet problem for hyperbolic systems in a wedge, Algebra i Analiz 11 (1999), no. 3, 140-195; English transl., St. Petersburg Math. J. 11 (2000), no. 3, 497-534. MR1711368 (2000j:35170)

[3] - On the asymptotics of solutions to the Neumann problem for hyperbolic systems in domains with conical points, Algebra i Analiz 16 (2004), no. 3, 56-98; English transl., St. Petersburg. Math. J. 16 (2005), no. 3, 477-506. MR2083566 (2005k:35248)

[4] A. Yu. Kokotov, P. Neŭttaämaki, and B. A. Plamenevskiŭ, The Neumann problem for the wave equation in a cone, Probl. Mat. Anal., vyp. 20, "Nauchn. Kniga", Novosibirsk, 2000, pp. 71-110; English transl., J. Math. Sci. 102 (2000), no. 5, 4400-4428. MR.1807064 (2002k:35180)

[5] _ Problems of diffraction by a cone: Asymptotic behavior of the solutions near the vertex, Zap. Nauchn. Sem. S.-Peterburg. Otdel. Mat. Inst. Steklov. (POMI) 259 (1999), 122-144; English transl., J. Math. Sci. 109 (2002), no. 5, 1894-1910. MR1754360 (2001m:35195)

[6] V. A. Kondrat'ev and O. A. Oleŭnik, Boundary value problems for a system in elasticity theory in unbounded domains. Korn inequalities, Uspekhi Mat. Nauk 43 (1988), no. 5, 55-98; English transl., Russian Math. Surveys 43 (1988), no. 5, 65-119. MR971465 (89m:35061)

[7] S. Agmon, Problèmes mixtes pour les équations hyperboliques d'ordre supérieur, Les Équations aux Dérivées Partielles (Paris, 1962), Centre Nat. Rech. Sci., Paris, 1963, pp. 13-18. MR0168933 $(29: 6189)$

[8] H.-O. Kreiss, Initial boundary value problems for hyperbolic systems, Comm. Pure. Appl. Math. 23 (1970), 277-298. MR0437941 (55:10862)

[9] R. Sakamoto, Mixed problems for hyperbolic equations. I, J. Math. Kyoto Univ. 10 (1970), 349-373. MR 0283400(44:632a)

[10] M. S. Agranovich, Boundary value problems for systems with a parameter, Mat. Sb. (N.S.) 84 (1971), no. 1, 27-65; English transl., Math. USSR-Sb. 13 (1971), 25-64. MR0285808 (44:3025)

[11] L. R. Volevich and S. G. Gindikin, Mixed problem for partial differential equations with quasihomogeneous principal part, "Editorial URSS", Moscow, 1999; English transl. from the Russian manuscript, Transl. Math. Monogr., vol. 147, Amer. Math. Soc., Providence, RI, 1996. MR1357662 (96j:35145)

[12] L. Gårding, Le problème de la dérivée oblique pour l'équation des ondes, C. R. Acad. Sci. Paris Sér. A-B 285 (1977), A773-A775 (Rectification, C. R. Acad. Sci. Paris Sér. A-B 286 (1978), A1199). MR 0458511 (56:16711) MR0501113(80a:35067)

[13] L. Hörmander, The analysis of linear partial differential operators. III. Pseudodifferential operators, Grundlehren Math. Wiss., vol. 274, Springer-Verlag, Berlin, 1985. MR781536 (87d:35002a)

[14] V. G. Maz'ya and B. A. Plamenevskiru, The coefficients in the asymptotics of the solutions of elliptic boundary value problems in a cone, Zap. Nauchn. Sem. Leningrad. Otdel. Mat. Inst. Steklov. (LOMI) 52 (1975), 110-127; English transl., J. Soviet Math. 9 (1978), no. 5, 750-764. MR0407445 (53:11220)

[15] _ $L_{p}$-estimates of solutions of elliptic boundary value problems in domains with ribs, Trudy Moskov. Mat. Obshch. 37 (1978), 49-93; English transl. in Trans. Moscow Math. Soc. 1980, no. 1. MR514327 (81b:35027)

[16] Weighted spaces with inhomogeneous norms, and boundary value problems in domains with conical points, Elliptische Differentialgleichungen (Meeting, Rostock, 1977), Wilhelm-Pieck-Univ., Rostock, 1978, pp. 161-190. (Russian) MR540196 (81e:35045)

[17] S. A. Nazarov and B. A. Plamenevskiú, Elliptic problems in domains with piecewise smooth boundaries, "Nauka", Moscow, 1991; English transl., de Gruyter Exp. Math., vol. 13, Walter de Gruyter, Berlin, 1994. MR.1283387 (95h:35001)

[18] G. Eskin, The wave equation in a wedge with general boundary conditions, Comm. Partial Differential Equations 17 (1992), no. 1-2, 99-160. MR1151258 (92m:35152)

[19] J. Cheeger and M. Taylor, On the diffraction of waves by conical singularities. I, Comm. Pure Appl. Math. 35 (1982), no. 3, 275-331. MR649347(84h:35091a)

[20] - On the diffraction of waves by conical singularities. II, Comm. Pure Appl. Math. 35 (1982), no. 4, 487-529. MR657825 (84h:35091b)

[21] M. Uchida, Microlocal analysis of diffraction by a corner, Ann. Sci. École Norm. Sup. (4) 25 (1992), no. 1, 47-75. MR1152613 (93b:35004)

[22] P. Gérard and G. Lebeau, Diffusion d'une onde par un coin, J. Amer. Math. Soc. 6 (1993), no. 2, 341-424. MR 1157289 (93f:35130) 
[23] P. Grisvard, Contrôlabilité exacte des solutions de l'équation des ondes en présence de singularités, J. Math. Pures Appl. (9) 68 (1989), 215-259. MR1010769 (90i:49045)

[24] V. A. Borovikov, Diffraction by polygons and polyhedra, "Nauka", Moscow, 1966. (Russian) MR0218058 (36:1147)

[25] V. B. Poruchikov, Methods of the dynamic theory of elasticity, "Nauka", Moscow, 1986. (Russian) MR 846431 (87g:73033)

[26] V. A. Dobrushkin, Boundary value problems of the dynamic theory of elasticity for wedge-shaped domains, "Nauka i Tekhnika", Minsk, 1988. (Russian) MR993072 (91e:73023)

[27] I. I. Mel'nikov, Singularities of the solution of a mixed problem for second-order hyperbolic equations in domains with a piecewise-smooth boundary, Uspekhi Mat. Nauk 37 (1982), no. 1, 149-150; English transl., Russian Math. Surveys 37 (1982), no. 1, 168-169. MR643778 (84j:35106)

[28] Nguyen Manh Hung, Asymptotics of solutions of the first boundary value problem for strongly hyperbolic systems near a conical point of the boundary of a domain, Mat. Sb. 190 (1999), no. 7, 103-126; English transl., Sb. Math. 190 (1999), no. 7, 1035-1058. MR.1725214 (2000m:35117)

[29] S. I. Matyukevich, On the nonstationary Maxwell system in domains with edges, Algebra i Analiz 15 (2003), no. 6, 86-140; English transl., St. Petersburg Math. J. 15 (2004), no. 6, 875-913. MR2044633 (2005d:35251)

[30] V. A. Kozlov and V. G. Maz'ya, Spectral properties of operator pencils generated by elliptic boundary value problems in a cone, Funktsional. Anal. i Prilozhen. 22 (1988), no. 2, 38-46; English transl., Funct. Anal. Appl. 22 (1988), no. 2, 114-121. MR947604 (90a:47125)

[31] V. A. Kozlov, V. G. Maz'ya, and J. Rossmann, Spectral problems associated with corner singularities of solutions to elliptic equations, Math. Surveys Monogr., vol. 85, Amer. Math. Soc., Providence, RI, 2001. MR.1788991 (2001i:35069)

[32] J.-L. Lions and E. Magenes, Problèmes aux limites non homogènes et applications. Vol. 1, Dunod, Paris, 1968. MR0247243(40:512)

St. Petersburg State University, Russia

E-mail address: matsi@math.nw.ru

St. Petersburg State University, Russia

E-mail address: plamen@rol.ru

Received 1/DEC/2005

Translated by B. A. PLAMENEVSKII 